\title{
Sobre \\ renormalização e rigidez quaseconforme \\ de polinômios quadráticos
}

Arcelino Bruno Lobato do Nascimento

\author{
DISSERTAÇÃO APRESENTADA \\ $\mathrm{AO}$ \\ Instituto de MatemáticA e EstatísticA \\ DA \\ Universidade DE SÃo PAUlo \\ PARA \\ OBTENÇÃO DO TÍTULO \\ $\mathrm{DE}$ \\ Mestre em CiÊnCIAS
}

Programa: Matemática

Orientador: Prof. Dr. Sylvain Philippe Pierre Bonnot

Durante o desenvolvimento deste trabalho o autor recebeu auxílio financeiro da CNPq

São Paulo, Março de 2016 


\title{
Sobre renormalização e rigidez quaseconforme de polinômios quadráticos.
}

\author{
Esta versão da dissertação contém as correções e alterações sugeridas \\ pela Comissão Julgadora durante a defesa da versão original do trabalho, \\ realizada em 01/08/2016. Uma cópia da versão original está disponível no \\ Instituto de Matemática e Estatística da Universidade de São Paulo.
}

Comissão Julgadora:

- Prof. Dr. Sylvain Philippe Pierre Bonnot (orientador) - IME-USP

- Prof. Dr. Edson Vargas - IME-USP

- Prof. Dr. Ali Messaoudi - UNESP-IBILCE 


\section{Agradecimentos}

Gostaria inicialmente de agradecer a todos aqueles que tornaram possível a realização deste trabalho.

Em especial agradeço:

À minha mãe Iady Lobato e meus irmãos Yara, Wellington e Claudete pelo amor e apoio constante. E à toda família Lobato!

Ao meu orientador Sylvain Bonnot por ser essa pessoa incrível. Paciente, amigo, incentivador e que sempre esteve disposto a me atender e me ensinar. Obrigado!

Ao professor Edison Vargas, com quem cursei a disciplina Dinâmica Complexa, por sua colaboração. Ao Arlane que me orientou durante a graduação, que me incentivou a vir estudar no IME. "Lembrome do dia em que o Arlane foi a sala do professor Arthur perguntar-me se eu estava querendo estudar Matémática, já me dando um artigo para eu lhe apresentar no dia seguinte!(Demorei mais de um dia pra entendê-lo!) Obrigado por tudo!"

Ao professor Arthur, que foi meu professor no Liceu Maranhense, pelo apoio, colaboração e crédito que sempre depositou em mim. Grande incentivador.

Agradeço também ao amigos no IME: Salvador, German, Belmiro, Bruna, Larissa, Lorena, Simone, Raibel, Diego, Diego Rugev,...

Aos meu amigos da UFMA que estudam no IME: Elivaldo e Jailson.

À Ana Kelly, minha amiga desde a UFMA, por quem tenho grande admiração.

À Luna Lomonaco por nossas breves, mas proveitosas discussões sobre dinâmica complexa.

À CNPq pelo apoio financeiro desde a iniciação científica pelo Instituto do Milênio/IMPA o que decerto me direcionou até aqui. 


\section{Resumo}

do NASCIMENTO, Arcelino Bruno Lobato. Sobre renormalização e rigidez quaseconforme de polinômios quadráticos. 2016. 92 f. Dissertação (Mestrado) - Instituto de Matemática e Estatística, Universidade de São Paulo, São Paulo, 2016.

Sem dúvida a questão central em Dinâmica Holomorfa é aquela sobre a densidade de hiperbolicidade. Temos a seguinte conjectura devida a Pierre Fatou: No espaço das aplicaçôes racionais de grau d o conjunto das aplicaçôes racionais hiperbólicas neste espaço formam um subconjunto aberto $e$ denso. Nem mesmo para a família dos polinômios quadráticos esta quetão foi respondida. Para a família quadrática este problema é equivalente a mostrar a não existência de polinômios quadráticos que suportam sobre o seu conjunto de Julia um campo de linhas invariante. Devido a reultados de Jean-Christophe Yoccoz sabemos da não existência de campos de linhas invariante para polinômios quadráticos no máximo finitamente renormalizáveis. Nesta dissertação é mostrado que um polinômio quadrático infinitamente renormalizável satisfazendo certa hipótese geométrica, denominada robustez, não suporta sobre o seu Julia um campo de linhas invariante. Esta prova foi obtida por Curtis T. McMullen e publicada em [McM1]. Os avanços na teoria de renormalização e quanto ao problema da densidade de hiperbolicidade e problemas relacionados tem contado com a colaboração de inúmeros renomados matemáticos como Mikhail M. Lyubich, Artur Ávila, Mitsuhiro Shishikura, Curtis T. McMullen, Jean-Christophe Yoccoz, Sebastien van Strien, Hiroyuki Inou, dentre outros.

Palavras-chave: família quadrática, renormalização, rigidez. 


\section{Abstract}

do NASCIMENTO, Arcelino Bruno Lobato. On renormalization and quasiconformal rigidity of quadratic polynomials. 2016. 92 f. Dissertation (Graduate) - Instituto de Matemática e Estatística, Universidade de São Paulo, São Paulo, 2010.

Undoubtedly one of the central open questions in Holomorphic Dynamics is about proving the density of hyperbolicity. That question was first raised by Pierre Fatou:In the space of rational functions of degree $d$ the set of hyperbolic rational functions form a open and dense subset. Not even for the family of quadratic polynomials this question been answered. For this particular quadratic family the problem is equivalent to showing the non-existence of quadratic polynomial with a Julia set supporting an invariant line field. Due to results by Jean-Christophe Yoccoz we already know the non-existence of invariant line fields for the quadratic polynomials that are at most finitely renormalizable. In this dissertation it is shown that an infinitely renormalizable quadratic polynomial satisfying a certain geometric hypotesis, called robustness, does not have an invariant line field supported on its Julia set. This proof was obtained by Curtis T. McMullen and published in [McM1]. Many advances on the theory of renormalization and on the problem of density of hyperbolicity have been already accomplished through the collective work of several renowned mathematicians such as Mikhail M. Lyubich, Artur Ávila, Mitsuhiro Shishikura, Curtis T. McMullen, Jean-Christophe Yoccoz, Sebastien van Strien, Hiroyuki Inou among others.

Keywords: quadratic family, renormalization, rigidity. 


\section{Sumário}

$\begin{array}{ll}\text { Lista de Figuras } & \text { ix }\end{array}$

1 Introdução $\quad \mathbf{1}$

1.1 Sistemas Dinâmicos e Dinâmica Holomorfa . . . . . . . . . . . . . . . . . . 1

1.2 Renormalização e Rigidez . . . . . . . . . . . . . . . . . . . . 4

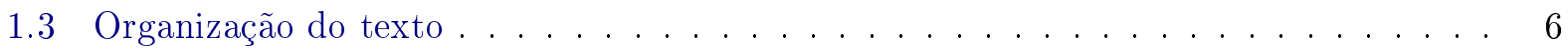

2 Elementos preliminares $\quad 9$

2.1 Dinâmica de polinômois quadráticos. . . . . . . . . . . . . . . . 9

$2.1 .1 \quad$ A esfera de Riemann . . . . . . . . . . . . . . . . . . . . . 10

2.1.2 Extensão de um polinômio quadrático $P_{c}=z^{2}+c$ à $\widehat{\mathbb{C}} \ldots \ldots \ldots$

2.1 .3 A família quadrática . . . . . . . . . . . . . . . . . . . 11

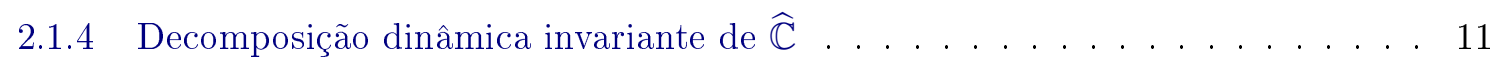

2.1.5 O conjunto de Mandelbrot . . . . . . . . . . . . . . . . . . . . . . . . 14

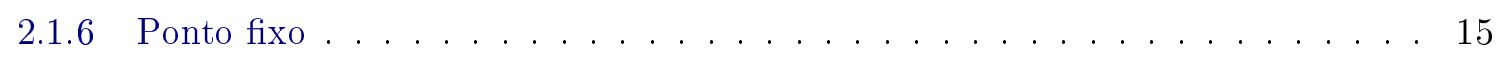

2.1.7 Raios externos e equipotenciais . . . . . . . . . . . . . . . 16

2.1 .8 Pontos fixos de $P_{c} \ldots \ldots \ldots \ldots \ldots \ldots \ldots$

2.1 .9 O quebra-cabeça de Yoccoz. . . . . . . . . . . . . . . . . 18

2.2 Aplicação do tipo polinomial . . . . . . . . . . . . . . . . . 19

2.3 Aplicação quaseconforme. . . . . . . . . . . . . . . . . . . . . . . 21

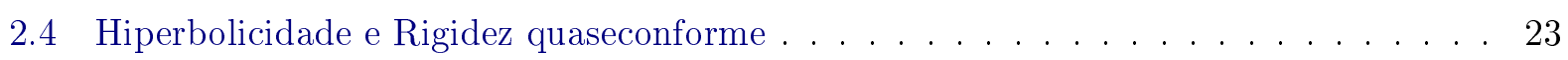

2.5 Definições e resultados gerais . . . . . . . . . . . . . . . . 27

2.5.1 Geodésicas fechadas em superfícies de Riemann hiperbólicas . . . . . . . . . 28

3 Renormalização $\quad 31$

3.1 Os pequenos conjuntos de Julia se intersectam em pontos periódicos. . . . . . . . . 36

3.2 Renormalização Simples . . . . . . . . . . . . . . . . . . . . 38

3.3 Tipos de Renormalizações . . . . . . . . . . . . . . . . . . . . . . . . 39

3.3 .1 Multiplicidade e Ramificação . . . . . . . . . . . . . . . . . . . . 41

3.4 Polinômios infinitamente renormalizáveis . . . . . . . . . . . . . . . . 41

$3.4 .1 \quad$ A tabela crítica . . . . . . . . . . . . . . . . . . . . 43

3.5 Medida e conectividade local . . . . . . . . . . . . . . . . . . . 45

$\begin{array}{lll}4 & \text { Robustez } & 47\end{array}$

4.0 .1 Área do conjunto pós-crítico . . . . . . . . . . . . . . . . . . . 49 
viii SUMÁRIO

5 Rigidez $\quad 53$

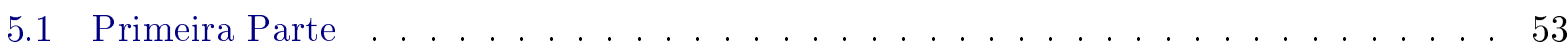

5.1 .1 Rigidez de aplicações do tipo polinomial . . . . . . . . . . . . . . . 54

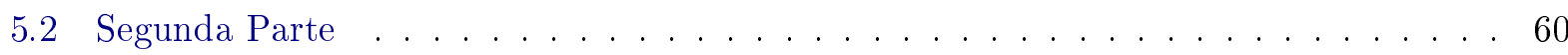

6 Considerações finais $\quad 73$

$\begin{array}{ll}\text { Referências Bibliográficas } & 75\end{array}$ 


\section{Lista de Figuras}

1.1 Conjunto de Mandelbrot . . . . . . . . . . . . . . . . . . . . . . . . . 2

1.2 À esquerda em cinza o conjunto $A_{\infty}(c)$; em preto o Julia cheio e à direita o conjunto de Julia correspondente. . . . . . . . . . . . . . . . . . . 3

1.3 O conjunto de Mandelbrot no centro e alguns Julia cheios com seus parâmetros indicados. . . . . . . . . . . . . . . . . . . .

1.4 Conjunto de Mandelbrot à esquerda e, à direita, zoom na parte enquadrada no Mandelbrot com o centro da componente hiperbólica de período 6 marcado. . . . . . . . . 4

1.5 Acima Julia cheio do polinômio $P_{c} \operatorname{com} c=-1.77289290338162 \ldots$; abaixo, o Julia cheio de $P^{3}$ à esquerda e à direita o Julia cheio de $P^{6} \ldots \ldots \ldots \ldots \ldots$

1.6 Acima Julia cheio do polinômio $P_{c} \operatorname{com} c=-1.77289290338162 \ldots$; abaixo, o Julia cheio de $P^{3}$ no centro e as suas pequens cópias. . . . . . . . . . . . . 5

2.1 Esfera de Riemann . . . . . . . . . . . . . . . . . . . . . . . . . . . 10

2.2 Julia cheio . . . . . . . . . . . . . . . . . . . . . . . . . . . . 12

2.3 Conjuntos de Julia . . . . . . . . . . . . . . . . . . . . . . . . . . . 12

2.4 Componentes do Fatou . . . . . . . . . . . . . . . . . . . . . . . . . 14

2.5 Conjunto de Mandelbrot . . . . . . . . . . . . . . . . . . . . . . . . . 15

2.6 Mandelbrot e um cópia sua. Zoom dado na região enquadrada na figura (a) . . . . . 15

2.7 laminação geodésica do disco . . . . . . . . . . . . . . . . . . . . 15

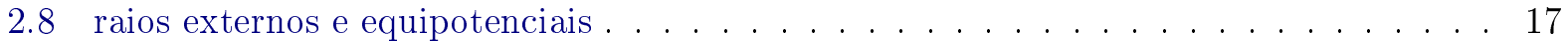

2.9 Quebra-cabeça de Yoccoz da basílica . . . . . . . . . . . . . . . . . . 18

2.10 Quebra-cabeça do Coelho de Douady-nível $0 \ldots \ldots$. . . . . . . . . . . . . . . . 19

2.11 Quebra-cabeça do Coelho de Douady-nível 1 . . . . . . . . . . . . . . . . . . . . 19

2.12 aplicação do tipo polinômial . . . . . . . . . . . . . . . . . . . . . . . 20

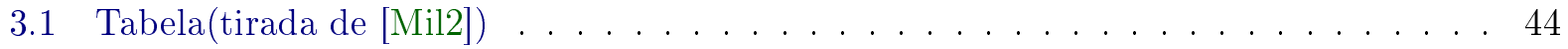

5.1 Construção do campo de linhas univalente $\nu \ldots \ldots \ldots \ldots \ldots \ldots$

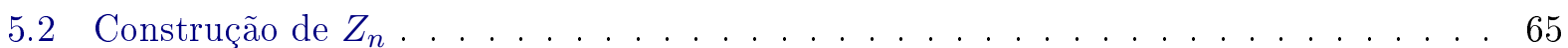

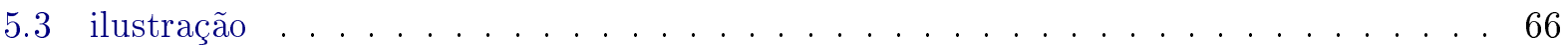




\section{Capítulo 1}

\section{Introdução}

Esta dissertação basea-se no livro Complex Dinamics and Renormalization de autoria do matemático Curtis T. McMullen no qual é provada parte de uma conjectura em Dinâmica Holomorfa conhecida como rigidez quaseconforme.

Mais especificamente, lidamos com um problema de rigidez restrito à família quadrática. Este problema pode ser enunciado da seguinte maneira:

Conjectura 1.0.1. Um polinômio quadrático não suporta sobre o seu conjunto de Julia um campo de linhas invariante.

No referido livro, Curtis T. McMullen provou o seguinte:

Teorema 1.1 (McMullen-1994). Um polinômio quadrático infinitamente renormalizável robusto não suporta sobre o seu conjunto de Julia um campo de linhas invariante.

Este teorema é o resultado principal desta dissertação.

McMullen provou ainda que todo polinômio quadrático infinitamente renormalizável $P_{c}$ com $c \in \mathcal{M} \cap \mathbb{R}$ é robusto. E disto, mais um resultado provado por Yoccoz(veja o enunciado logo abaixo), concluiu que os polinômios $P_{c} \operatorname{com} c \in \mathcal{M} \cap \mathbb{R}$ não suportam sobre o seu Julia um campo de linhas invariante.

Teorema 1.2 (Yoccoz). Um polinômio quadrático que suporta sobre o seu Julia um campo de linhas invariante é infinitamente renormalizável.

Este trabalho apresenta um estudo sobre renormalização de polinômios quadráticos e uma rápida introdução à dinâmica holomorfa e tópicos relacionados.

\subsection{Sistemas Dinâmicos e Dinâmica Holomorfa}

Os sistemas dinâmicos fornecem modelos excelentes para a descrição qualitativa e quantitativa de sistemas em evolução no âmbito das ciências experimentais. Uma primeira classificação dos sistemas dinâmicos os divide entre sistemas dinâmicos discretos e sistemas contínuos. O principal objetivo da área consiste em descrever o comportamento futuro de um sistema, estudando principalmente objetos e quantidades invariantes durante a evolução. Em geral, esses sistemas dinâmicos dependem de parâmetros. 
Dentro da área de Sistemas Dinâmicos, a Dinâmica Holomorfa ocupa um lugar muito especial. Esta área de pesquisa é muito recente, desde que passou por um renascimento nos anos oitenta, e tem tido um crescimento explosivo nas últimas décadas.

Deste modo, em geral, em Dinâmica Holomorfa unidimensional estamos interessados em analisar o comportamento de uma função holomorfa agindo por iterações em uma Superfície de Riemann, como, por exemplo, entender a distribuição da órbita de um ponto, ver a existência de conjuntos invariantes e descrever sua topologia e geometria.

A área da Dinâmica Holomorfa é bem conhecida por ter feito popular a noção de conjuntos fractais. Estes são objetos que apresentam frequentemente um padrão de autossemelhança e uma dimensão de Hausdorff que não é um número inteiro. O trabalho de Benoit Mandelbrot $(1924$ - 2010) mostrou o quanto onipresentes esses objetos são. Graças aos trabalhos pioneiros produzidos por Adrien Douady(1935 - 2006) e John Hamal Hubbard(1945), muitas das conjecturas feitas por Mandelbrot foram rigorosamente demonstradas, iniciando assim uma nova era na dinâmica holomorfa.

Atualmente a maior parte das pesquisas em dinâmica holomorfa giram em torno da dupla plano dinâmico - plano dos parâmetros. Ou seja, sistemas dinâmicos são descritos não como objetos únicos, mas como variando dentro de famílias de tais objetos. Muitas vezes, as características dinâmicas que aparecem no plano dinâmico são refletidas dentro do plano dos parâmetros. Isto quer dizer que podemos obter informações importantes sobre a topologia, por exemplo, do plano dos parâmetros através do estudo das variações que acontecem no plano dinâmico quando os parâmetros são movidos: "You first plow in the dynamical plane and then harvest in the parameter plane."(Adrien Douady)

O espaço de parâmetros mais conhecido é, sem dúvidas, o conjunto de Mandelbrot $\mathcal{M}$, que é o espaço de parâmetros para a família dos polinômios quadráticos $P_{c}: \mathbb{C} \longrightarrow \mathbb{C}$ definidos por $P_{c}: z \longrightarrow z^{2}+c$, onde o parâmetro $c$ é um número complexo.

O conjunto de Mandelbrot $\mathcal{M}$ pode ser definido como

$$
\mathcal{M}:=\left\{c \in \mathbb{C} ; \text { a órbita de } 0 \text { sob iteração de } P_{c} \text { é limitada }\right\} \text {. }
$$

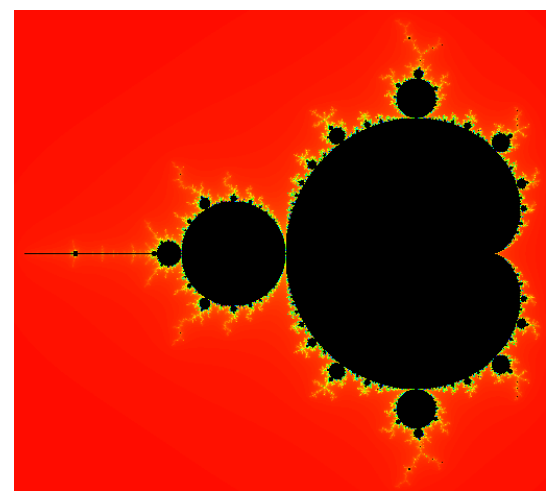

Figura 1.1: Conjunto de Mandelbrot

A intrincada estrutura da família dos polinômios quadráticos foi revelada através dos trabalhos de A. Douady e J. H. Hubbard([DH1], [DH2], [DH3], [Dou]).

Para cada membro da família quadrática $P_{c}(z)=z^{2}+c$, (bem como para qualquer polinômio sobre $\widehat{\mathbb{C}})$ o ponto $\infty$ é um ponto fixo super-atrator, isto é, $\infty$ é um ponto fixo de $P_{c}$ para o qual 
também é ponto crítico. Denominamos $A_{\infty}(c)$ a sua bacia de atração, ou seja, $A_{\infty}(c):=\{z \in$ $\widehat{\mathbb{C}} ; P_{c}^{n}(z) \rightarrow \infty$ quando $\left.n \rightarrow \infty\right\}$. Chamaremos de conjunto de Julia cheio $K(c)$ do polinômio $P_{c}$ o conjunto de pontos que possuem órbita uniformemente limitada em $\mathbb{C}$, assim $K(c)=\widehat{\mathbb{C}} \backslash A_{c}(\infty)$. O conjunto de Julia $J(c)$ do polinômio $P_{c}$ é a fronteira comum do conjunto cheio de Julia(Julia cheio) e a bacia de atração do infinito, isto é, $J(c)=\partial K(c)=\partial A_{c}(\infty)$. Um Teorema clássico provado por Fatou em 1918 afirma que o conjunto de Julia cheio de um polinômio $P$ é conexo se, e somente se, ele contém todos os pontos críticos finitos do polinômio, enquanto se $P$ tem pelo menos um ponto crítico finito no complementar de $K(P)$, isto é, na bacia do infinito, então $J(P)$ e $K(P)$ possuem uma quantidade não enumerável de componentes conexas (consulte [Mil1]). No caso quadrático, $P_{c}$ tem apenas um ponto crítico finito, a saber, o ponto $z=0$. Neste caso, ou $0 \in K(c)$ e, portanto, $K(c)$ é conexo, ou $0 \notin K(c)$ e, então, $K(c)$ é um conjunto de Cantor. O conjunto de Mandelbrot $\mathcal{M}$ também pode ser definido como o conjunto de parâmetros $c \in \mathbb{C}$ para os quais $K(c)$ é conexo (veja Figura 1.3).

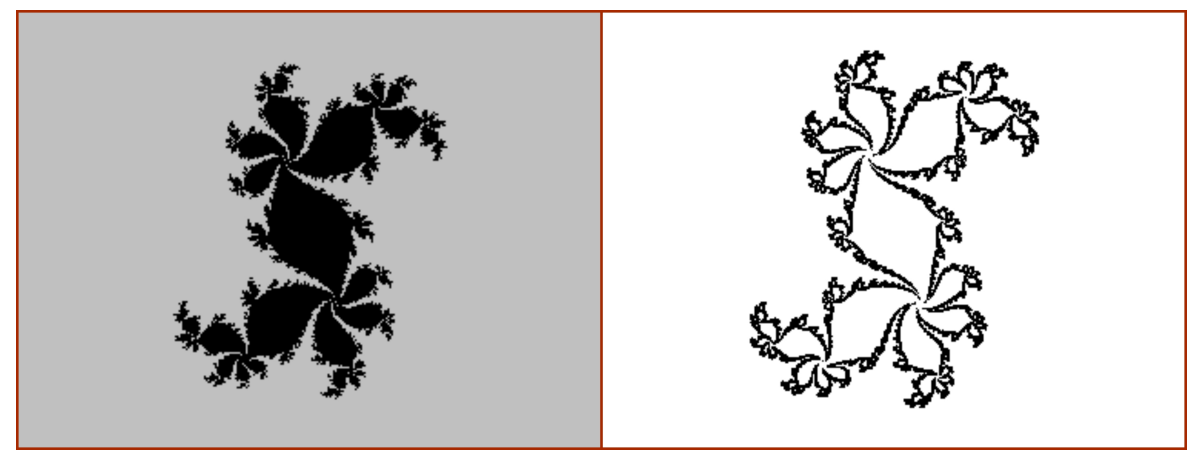

Figura 1.2: $\grave{A}$ esquerda em cinza o conjunto $A_{\infty}($ c); em preto o Julia cheio e à direita o conjunto de Julia correspondente.

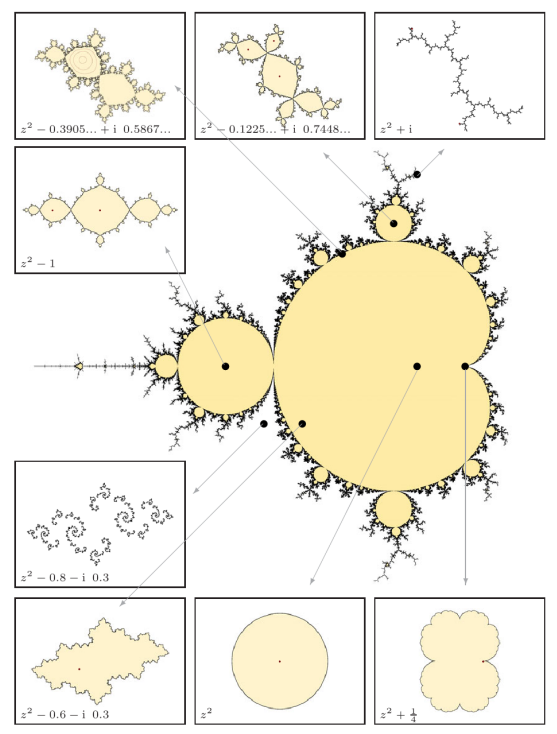

Figura 1.3: $O$ conjunto de Mandelbrot no centro e alguns Julia cheios com seus parâmetros indicados. 


\subsection{Renormalização e Rigidez}

O objetivo central deste trabalho é entender o comportamento dos iterados elevados de um polinômio quadrático $P_{c}$, isto é, compreender o comportamento de $P_{c}^{n}$ para $n$ muito grande.

Uma característica marcante de sistemas dinâmicos de baixa dimensão é que o seu comportamento a longo prazo em uma escala espacial menor é descrito por um sistema dinâmico induzido que pertence a mesma classe do sistema dinâmico inicial.

Assim, um procedimento de renormalização (ou um operador de renormalização) é definido considerando-se os sistemas dinâmicos induzidos depois de uma mudança de coordenadas conveniente (renormalização). Para o caso quadrático, com o qual trabalhamos, tal mudança de coordenadas se dá pelo seguinte fundamental teorema em dinâmica holomorfa e teoria de renormalização devido à A. Douady e John H. Hubbard.

Teorema 1.3 (Teorema de retificação (Straightening theorem) - caso quadrático). Toda aplicação do tipo quadrático é hibridamente equivalente a um polinômio quadrático $P_{c}$. Se c $\in \mathcal{M}$, então $P_{c}$ é único.

Para uma definição de aplicação do tipo quadrático e equivalência híbrida consulte as seções 2.2 e 2.4 .

Definição 1.1. Seja $P_{c}(z)=z^{2}+c$ um polinômio quadrático com seu conjunto de Julia conexo. $A$ aplicação $P_{c}^{n}$ é renormalizável se existem discos topológicos $U$ e $V$ em $\mathbb{C}$ com $0 \in U \subset V$ e $P_{c}^{n}: U \rightarrow V$ é uma aplicação do tipo quadrático com Julia conexo. Dizemos ainda que $P_{c}$ é renormalizável.

Exemplo 1.1. Consideremos o polinômio $P(z)=z^{2}-1.77289290338162 \ldots$.

$c=-1.77289290338162 \ldots$ é o centro da componente hiperbólica de perído 6 do conjunto de Mandelbrot(veja a figura 3 ) e, portanto, $P^{6}(0)=0 . P^{3}$ e $P^{6}$ são renormalizáveis. O ponto crítico $z=0$ é periódico de período 2 para a aplicação $P^{3}$, em razão disto o Julia cheio renormalizado de $P$ será o Julia cheio do polinômio quadrático $P_{-1}: z \mapsto z^{2}-1$ para o qual $z=0$ é periódico de período 2. Enquanto para $P^{6}$ o ponto $z=0$ é fixo e, portanto, o polinômio renormalizado de $P^{6}$ será $P_{0}: z \mapsto z^{2}$.

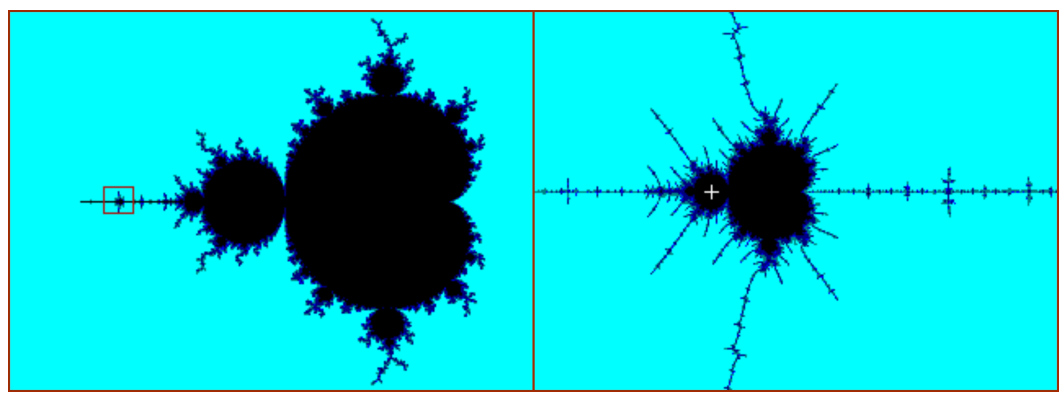

Figura 1.4: Conjunto de Mandelbrot à esquerda e, à direita, zoom na parte enquadrada no Mandelbrot com o centro da componente hiperbólica de período 6 marcado. 


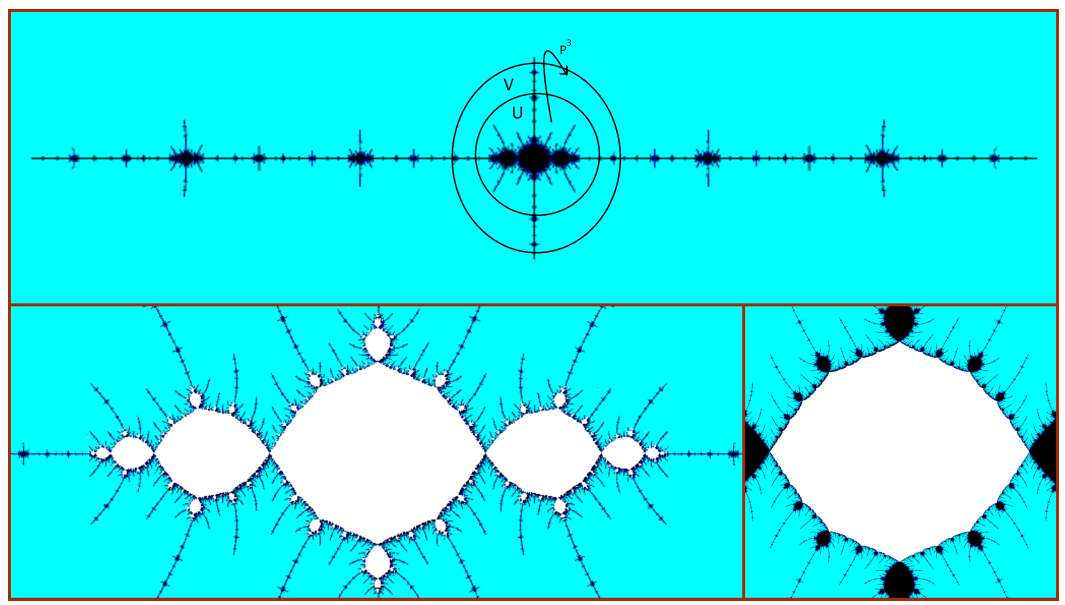

Figura 1.5: Acima Julia cheio do polinômio $P_{c} \operatorname{com} c=-1.77289290338162 \ldots$; abaixo, o Julia cheio de $P^{3}$ à esquerda e à direita o Julia cheio de $P^{6}$

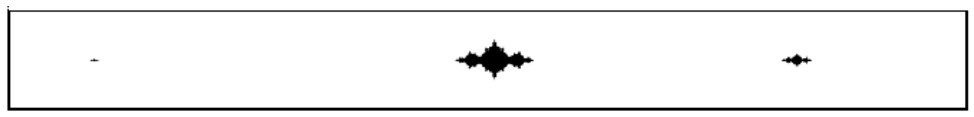

Figura 1.6: Acima Julia cheio do polinômio $P_{c}$ com $c=-1.77289290338162 \ldots$; abaixo, o Julia cheio de $P^{3}$ no centro e as suas pequens cópias.

O fenômeno da rigidez em suas diversas ocorrências consiste em uma das mais notáveis descobertas em geometria e sistemas dinâmicos de baixa dimensão. Ocorre em várias situações quando uma equivalência fraca entre certos objetos implica uma equivalência forte.

Pressupõe-se que a atenção aos problemas de rigidez se iniciou com a aparição do Teorema da rigidez de Mostow na geometria hiperbólica. Este teorema afirma que duas variedades hiperbólicas de dimensão 3 que são homotopicamente equivalentes são na verdade isométricas.

A rigidez na dinâmica holomorfa se apresenta em mais de uma forma. Nesta dissertação estamos lidando com o seguinte problema, mas restrito aos polinômios quadráticos:

Conjectura 1.2.1. Uma aplicação racional $f: \widehat{\mathbb{C}} \longrightarrow \widehat{\mathbb{C}}$ não suporta sobre seu conjunto de Julia um campo de linhas invariante ou é duplamente recoberto por um endomorfismo integral do toro complexo.

Definição 1.2. Uma aplicação racional não constante de grau $d, f: \widehat{\mathbb{C}} \rightarrow \widehat{\mathbb{C}}$, é uma aplicação holomorfa da forma $f(z)=\frac{P(z)}{Q(z)}$ em que $P(z)$ e $Q(z)$ são polinômios sem fatores em comum e $d:=$ máx $\{\operatorname{grau}(P), \operatorname{grau}(Q)\}$, em que grau $(P)$ é o grau do polinômio $P$.

Na verdade é sabido que toda aplicação holomorfa $f: \widehat{\mathbb{C}} \rightarrow \widehat{\mathbb{C}}$ é uma aplicação racional $f(z)=$ $\frac{P(z)}{Q(z)}$ com $P(z)$ e $Q(z)$ unicamente determinados a menos de multiplicação por uma constante.

No caso quadrático tal conjectura corresponde à Conjectura 1.0.1. Ela é equivalente a um problema central em dinâmica holomorfa enunciado por Pierre Fatou(1878 - 1929), que permanece em aberto, e afirma que os polinômios quadráticos hiperbólicos, isto é, aqueles para os quais o ponto crítico $z=0$ tende para um ciclo atrator, formam um subconjunto aberto e denso da família quadrática.

Intuitivamente, um campo de linhas $\mu$ suportado no conjunto de Julia $J$ corresponde a considerar uma reta real $L(z)$ que passa pela origem do espaço tangente de cada ponto $z \in E \subset J$ de um 
subconjunto $E \subset J$ de $J$ de medida (de Lebesgue) positiva de modo que a inclinação destas retas variem mensuravelmente em relação à $z \in E$. Fora do subconjunto $E, L(z)$ é tomada como uma linha horizontal. Dizemos que um campo de linhas é invariante se $f^{-1}(E)=E$ e $f^{\prime}$ transforma a linha em $z$ na linha $f(z)$. Observe que, por definição, um conjunto de Julia de medida zero não suporta sobre si um campo de linhas. Em todo o texto, sempre que nos referirmos a medida estaremos nos referindo a medida de Lebesgue.

Entender as iteradas elevadas de uma aplicação racional $f$ de grau $d>1$ pode ser um problema bastante difícil. Existe uma tensão na dinâmica entre fatores expansores da $f$ como, por exemplo, o fato de seu grau tender para o infinito por iteração, e fatores contrativos tal como a presença de ponto crítico. As aplicações melhor compreendidas são as hiperbólicas, para as quais a tensão se reduz à concentração da expansão no conjunto de Julia da aplicação e à presença de contração no resto da esfera de Riemann[McM1].

Depois do Teorema 1.2 o foco sobre o problema de rigidez transferiu-se para o âmbito dos polinômios infinitamente renormalizáveis. Para tais polinômios, as propriedades expansoras e contrativas encontram-se em um delicado equilíbrio. Por exemplo, o ponto crítico $z=0$ pertence ao conjunto de Julia e sua órbita positiva é recorrente, isto é, possue subsequência que converge para 0. Além disso, os iterados futuros de $f$ para tempos arbitrariamente grandes podem ser renormalizados para se obter um novo sistema dinâmico com a mesma forma geral da aplicação original $f$. A repetição desta forma em um número infinito de escalas consiste no contexto básico deste estudo([McM1]).

Métodos da Teoria Geométrica das Funções, Aplicações Quaseconformes, Geometria Hiperbólica e Teoria dos espaços de Teichmüller tém contribuído significativamente para o aprofundamento e alcance das pesquisas nesta área. Sob hipóteses geometricas adicionais, mostraremos que os sistemas dinâmicos renormalizados variam em uma família compacta. A compacidade é estabelecida combinando estimativas universais para a geometria de superfícies hiperbólica com teoremas sobre distorção para aplicações holomorfas (vide:cap5-[McM1]). Com estas informações, se estabele a rigidez quaseconforme do polinômio original $P_{c}$. Ao longo do argumento principal vários aspectos da dinâmica complexa são envolvidos e outros são obtidos.

\subsection{Organização do texto}

Diferentemente da situação de um "paper", essencialmente todo arcabouço preliminar para a prova do teorema de rigidez 1.1, incluindo os resultados mais técnicos, são apresentados em [McM1]. No entanto, o presente trabalho desenvolve-se a partir dos capítulos 7, 8, 9 e 10 de [McM1], de modo que os resultados presentes nos capítulos iniciais que se fizerem necessários serão devidamente referenciados. Isto é feito para que não tenhamos um texto muito longo e cansativo.

O texto está organizado da seguinte maneira.

No Capítulo 2 introduzimos alguns aspéctos da dinâmica dos polinômios quadráticos, apenas o suficiente para o que será tratado aqui e para dar uma boa contextualização ao leitor. Nas seção 2.2 apresentamos as aplicações do tipo polinômial e as aplicações quaseconformes. Na seção 2.4 introduzimos alguns conceito de fundamental importância na Dinâmica Holomorfa os relacionando e discorrendo mais precisamente sobre rigidez quaseconforme. Por fim, listamos algumas definições e resultados gerais que seram úteis nos capítulos seguinte.

O Capítulo 3 destina-se à renomalização de polinômios quadráticos. Inúmeros resultados são 
obtidos nos assegurando, assim, uma boa descrição deste fenômeno, o que, por conseguinte faz deste capítulo uma boa introdução ao assunto. Apresentamos, também, neste capítulo uma ferramenta combinatória desenvolvida por Bodil Branner e John H. Hubbard denominada Tabela Crítica (Critical Tableau).

No Capítulo 4 introduzimos a noção de robustez para polinômios quadráticos infinitamente renormalizável e obtemos alguns resultados interessantes.

E finalmente, no Capítulo 5 provamos o teorema 1.1. Nas conciderações finais falamos sobre generalizações obtidas, indicando, portanto, os caminhos que têm sido tomados nos avanços desta teoria bem como aqueles envolvidos nestas conquistas. Indicamos, também, direções de possíveis generalizações. 


\section{Capítulo 2}

\section{Elementos preliminares}

\subsection{Dinâmica de polinômois quadráticos.}

Pressupõe-se que a dinâmica holomorfa tenha nascido com os estudos de Arthur Cayley (18211895) sobre o método de Newton no plano complexo por volta do ano 1879. No início do XX, Gaston Julia (1893 - 1978) e Pierre Fatou (1878 - 1929) iniciaram e desenvolveram um estudo sistemático de iterações de funções holomorfas na esfera de Riemann. No ano de 1942 C.L.Siegel colabora com o desenvolvimento desta área. A área passa por um tempo adormecida até que no início dos anos 80 a dinâmica holomorfa renasce com os trabalhos de Adrien Douady (1935 - 2006) e seu aluno John H. Hubbard (1945). Tal ressurgência se deve ao advento do computador, que possibilitou desenhar as bacias de atração e a visualisação do conjunto de Mandelbrot devida à Benoit Mandelbrot (1924 - 2010).

Definição 2.1 (função holomorfa). Seja $U \subset \mathbb{C}$ um conjunto aberto. Vamos dizer que uma função $f: U \longrightarrow \mathbb{C}$ é holomorfa em $z_{0} \in U$ se:

1. analiticidade: existe uma bola $B_{\epsilon}\left(z_{0}\right) \subset U$ de raio $\epsilon>0$ tal que $f$ nessa bola pode ser escrita como uma série de potências convergente:

$$
f(z)=\sum_{n \geq 0} a_{n}\left(z-z_{0}\right)^{n} \text { para todo } z \in B_{\epsilon}\left(z_{0}\right)
$$

isto é, $f$ é analítica em $z_{0}$, ou

2. $\mathbb{C}$-diferenciabilidade: existe o limite $\lim _{\zeta \rightarrow 0} \frac{f\left(z_{0}+\zeta\right)-f\left(z_{0}\right)}{\zeta}$. Denotamos este limite, quando existe, por $f^{\prime}\left(z_{0}\right)$ e o denominamos derivada de $f$ em $z_{0}$.

$f$ é holomorfa em $U$ se for holomorfa em cada ponto $z$ de $U$. Quando $a_{1} \neq 0$ ou $f^{\prime}\left(z_{0}\right) \neq 0$ diremos que $f$ é conforme em $z_{0}$. $f$ é conforme no aberto $U$ se for conforme em cada ponto de $U$. Quando $f$ tem uma inversa $f^{-1}$ e esta é também holomorfa dizemos que $f$ é biholomorfa ou é um biholomorfismo sobre $f(U)$.

Da Análise Complexa sabemos que 1. e 2. a cima são equivalentes.

Num sentido geral, em dinâmica holomorfa estamos interessados em entender o comportamento das iteradas de uma função holomorfa, isto é, $f^{\circ n}:=\underbrace{f \circ f \circ \cdots \circ f}_{n \text { vezes }}$. 


\subsubsection{A esfera de Riemann}

Consideremos o conjunto

$$
\widehat{\mathbb{C}}:=\mathbb{C} \bigcup\{\infty\},
$$

em que $\infty$ representa um ponto fora de $\mathbb{C}$.

Com a topologia $\tau:=\{U \subset \mathbb{C} ; U$ é aberto $\} \bigcup\{(\mathbb{C}-K) \cup\{\infty\} ; K \subset \mathbb{C}$ é compacto $\}, \widehat{\mathbb{C}}$ é um espaço topológico compacto Hausdorff homeomorfo à $\mathbb{S}^{2}$.

Podemos por em $\widehat{\mathbb{C}}$ uma estrutura holomorfa atravez do atlas holomorfo $\mathcal{A}:=\{(\mathbb{C} ; I d),(\mathbb{C}-$ $\{0\} \bigcup\{\infty\} ; \operatorname{Inv})\}$ em que:

- $\operatorname{Id}(z)=z$ para $z \in \mathbb{C}$;

- $\operatorname{Inv}(z)=\frac{1}{z}$ em $\mathbb{C}-\{0\}$ e $\operatorname{Inv}(\infty)=0$,

Definição 2.2. Seja $\Sigma$ a estrutura holomorfa que contém o atlas $\mathcal{A} . \widehat{\mathbb{C}}=(\widehat{\mathbb{C}} ; \Sigma)$ é uma superfície de Riemann, isto é, uma variedade complexa de dimensão 1, chamada Esfera de Riemann.

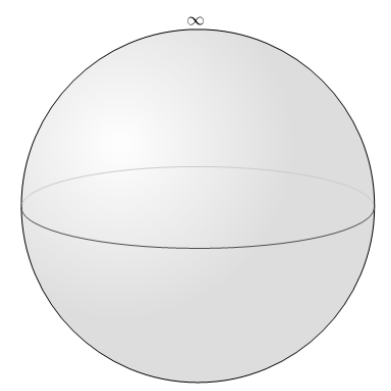

Figura 2.1: Esfera de Riemann

Definição 2.3 (Função holomorfa na esfera de Riemann). Uma função $f: \widehat{\mathbb{C}} \longrightarrow \widehat{\mathbb{C}}$ será holomorfa se $\left.f\right|_{\mathbb{C}}: \mathbb{C} \longrightarrow \mathbb{C}$ for holomorfa e $z \mapsto \frac{1}{f\left(\frac{1}{z}\right)}$ for holomorfa em uma vizinhança de 0 . Definimos a derivada de $f$ no infinito, $f^{\prime}(\infty)$, como sendo a derivada de sua representação local $z \mapsto \frac{1}{f\left(\frac{1}{z}\right)}$ no 0.

\subsubsection{Extensão de um polinômio quadrático $P_{c}=z^{2}+c \grave{a} \widehat{\mathbb{C}}$}

Definimos $P_{c}: \widehat{\mathbb{C}} \longrightarrow \widehat{\mathbb{C}}$ pondo $P_{c}=z^{2}+c$ se $z \in \mathbb{C}$ e $P_{c}(\infty)=\infty$.

É imediato que $P_{c}$ é holomorfa se $z \in \mathbb{C}$. Na variável, $w=\frac{1}{z}, P_{c}$ é dado numa vizinhança de 0 por

$$
w \mapsto \frac{w^{2}}{1+c w^{2}}
$$

Podemos tomar $z \in \widehat{\mathbb{C}}$ numa vizinhança do $\infty$ de modo que $\left|c w^{2}\right|<1$ e com isso teremos

$$
w \mapsto \frac{w^{2}}{1+c w^{2}}=w^{2}\left(1-c w^{2}+\cdots\right)=w^{2}-c w^{4}+\cdots,
$$

logo, $P_{c}$ é holomorfa no $\infty$ e, portanto, é holomorfa em $\widehat{\mathbb{C}}$. Note que $P_{c}^{\prime}(\infty)=0$, já que a expansão em séries de potências de sua representação local no infinito não possue o termo de grau 1. 


\subsubsection{A família quadrática}

A família quadrática é a classe de sistemas dinâmicos holomorfos que consideraremos neste trabalho. Estes sistemas dinâmicos são os que mais se estudou em Dinâmica Holomorfa e, no entanto, grandes perguntas permanecem sem respostas sobre eles. Apresentaremos nesta subseção apenas os conceitos que se faram necessários para o desenvolvimento do texto. Dessa forma, para mais informações vide bibliografia.

Definição 2.4. A Família Quadrática consiste na seguinte família a um parâmetro

$$
\mathcal{Q}:=\left\{P_{c} \in \operatorname{Hol}(\widehat{\mathbb{C}}, \widehat{\mathbb{C}}) ; P_{c}: z \mapsto z^{2}+c \quad \operatorname{com} \quad c \in \mathbb{C}\right\}
$$

O estudo dos polinômios $P_{c}$ é suficiente para compreendermos todos os polinômios quadráticos $P(z)=\alpha z^{2}+\beta z+\gamma \operatorname{com}(\alpha, \beta, \gamma) \in \mathbb{C}^{3}$ e $\alpha \neq 0$. Isto porque com uma mudança de variável todo polinômio quadrático $P(z)$ pode ser posto, de maneira única, na forma $P_{c}(z)=z^{2}+c$.

Definição 2.5. Dois polinômios quadráticos $P_{a}$ e $P_{b}$ são conformemente conjugados(equivalentes) se existe uma aplicação de Möbius $h: \widehat{\mathbb{C}} \longrightarrow \widehat{\mathbb{C}}$ tal que $P_{a}=h^{-1} \circ P_{b} \circ h$.

Proposição 2.1. Todo polinômio quadrático $P(z)=a z^{2}+2 b z+d$ é conformemente conjugado ao polinômio $P_{c}(z)=z^{2}+c$ com a conjugação $S(z)=a z+b$ e $c=a d+b-b^{2}$.

Vejamos então, um exemplo simples, mas que nos ajude a ter um intuição sobre a dinâmica destes polinômios.

Exemplo 2.1. Consideremos o polinômio $P_{0}=z^{2}$.

Escrevendo $z=r e^{2 \pi i t}$ teremos $P_{0}^{n}(z)=r^{2^{n}} e^{2^{n+1} \pi i t}$, e assim vê-se que:

1. $P_{0}^{n}(z) \rightarrow \infty$ se $r=|z|>1$;

2. $P_{0}^{n}(z) \rightarrow 0$ se $r=|z|<1$;

3. sobre o círculo $|z|=1, P_{0}$ é conjugada à $S(t)=2 t \bmod 1$. Se escrevermos $t$ na expansão diádica, $S$ corresponderá a aplicação deslocamento(shift). Com isso, trabalhando um pouco mais, conseguimos exibir um ponto em $\mathbb{S}^{1}$ cuja órbita por $P_{0}$ é densa em $\mathbb{S}^{1}$, isto é, $\overline{\left\{P_{0}^{n}\left(z_{0}\right) ; n \in \mathbb{N}\right\}}=\mathbb{S}^{1}$. E, portanto, a dinâmica de $P_{0}$ sobre $\mathbb{S}^{1}$ se apresenta mais interessante.

4. note que $P_{0}(0)=0 \operatorname{com} P_{0}^{\prime}(0)=0$ e $P_{0}(\infty)=\infty \operatorname{com} P_{0}^{\prime}(\infty)=0$

\subsubsection{Decomposição dinâmica invariante de $\widehat{\mathbb{C}}$}

A dinâmica de um polinômio qudrático particiona naturalmente a esfera de Riemann em dois subconjuntos complementares e totalmente invariantes, chamados conjuntos de Julia e Fatou. Pelo menos no contexto da dinâmica polinômial podemos dizer que o conjunto de maior interesse é o conjunto de Julia, pois é onde a dinâmica se apresenta mais complicada e interessante.

No caso polinomial esses conjuntos podem ser facilmente introduzidos a partir de um outro subconjunto invariante denominado conjunto cheio de Julia que em geral chamaremos apenas de Julia cheio. 
Definição 2.6 (Conjunto cheio de Julia). O conjunto cheio de Julia de $P_{c}: z \mapsto z^{2}+c$ é por definição o seguinte conjunto,

$$
K(c):=\left\{z \in \mathbb{C} ; \sup _{n}\left\{\left|P_{c}^{n}(z)\right| ; n \in \mathbb{N}\right\}<+\infty\right\}
$$

Definição 2.7 (Bacia do infinito). A bacia do infinito de $P_{c}$ é o subconjunto $\widehat{\mathbb{C}}-K(c)$, isto é,

$$
A_{\infty}(c):=\left\{z \in \widehat{\mathbb{C}} ; P_{c}^{n}(z) \rightarrow \infty \text { quando } n \rightarrow+\infty\right\}
$$
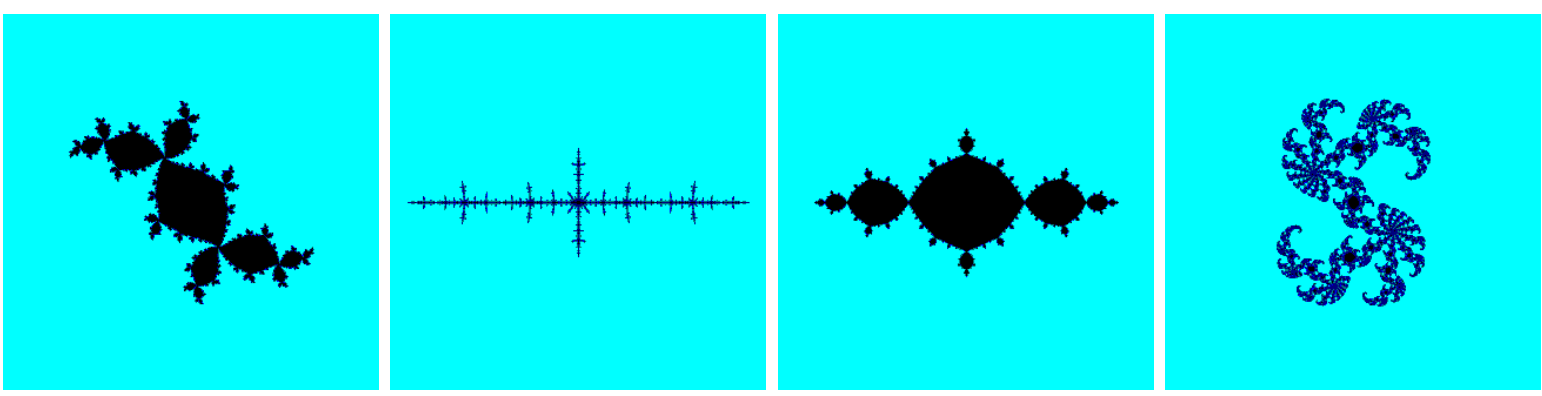

Figura 2.2: Julia cheio

Listamos a seguir algumas propriedades de $K(c)$ :

1. $K(c) \neq \varnothing$. Pois temos pelo menos os pontos fixos de $P_{c}$ em $K(c)$, isto é, contém os pontos $z_{0} \in \mathbb{C}$ tais que $P_{c}\left(z_{0}\right)=z_{0}$

2. $K(c)$ é compacto. Pois não é difícil ver que seu complementar contém uma vizinhança aberta do infinito;

3. $\widehat{\mathbb{C}}-K(c)$ é conexo. Decorre de uma aplicação direta do teorema do módulo máximo;

4. $K(c)$ é totalmente invariante, isto é, $P_{c}^{-1}(K(c))=K(c)$.

Definição 2.8. O conjunto de Julia de $P_{c}: z \mapsto z^{2}+c$ é por definição

$$
J(c):=\partial K(c)=\partial A_{\infty}(c)
$$

Veja a seguir alguns exemplos:

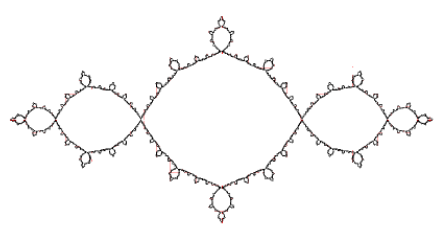

(a) $J(-1)$

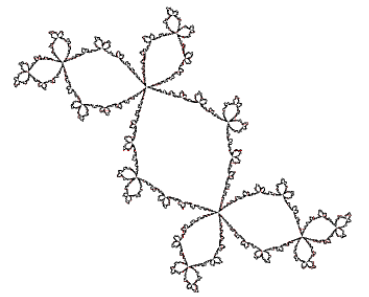

(b) $J(-0.123+i 0.744)$

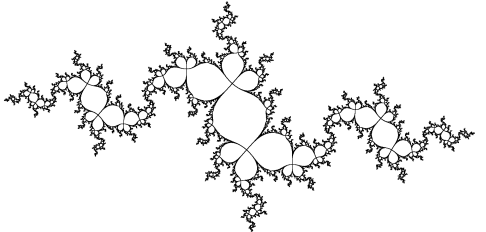

(c) basílica de coelhos

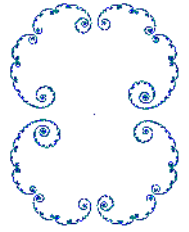

(d) $J\left(\frac{1}{4}+\epsilon\right)$

Figura 2.3: Conjuntos de Julia

Propriedades de $J(c)$ : 
1. $J(c) \neq \varnothing$

2. $J(c)$ é compacto;

3. $J(c)$ é totalmente invariante.

Definição 2.9 (Ponto crítico). Um ponto crítico para $P_{c}$ é um ponto $z \in \widehat{\mathbb{C}}$ tal que $P_{c}^{\prime}(z)=0$.

Muitas propriedades dinâmicas globais de $P_{c}$ dependem do comportamento do ponto crítico. Como é ilustrado pelo seguinte teorema devido a Fatou.

Teorema 2.1 (Fatou). Se $P_{c}^{n}(0) \rightarrow \infty$, então, $J(c)$ é totalmente disconexo.

Sabe-se que os conjuntos de Julia são subconjuntos perfeitos de $\widehat{\mathbb{C}}$, isto é, que todo ponto de $J(c)$ é ponto de acumulação de $J(c)$. A partir disso, temos a seguinte forte dicotomia:

Teorema 2.2. $J(c)$ é conexo ou um conjunto de Cantor.

Na figura 2.3(d) o conjunto de Julia é um Cantor.

Um outro conjunto que cumpre um papel importantíssimo nos sistemas dinâmicos holomorfos é o conjunto pós-crítico.

Definição 2.10. O conjunto pós-crítico de um polinômio quadrático $P_{c}$ é o conjunto

$$
\mathrm{P}(c):=\overline{\left\{P_{c}^{n}(0) ; n \geq 1\right\}}
$$

Da definição vê-se que $P_{c}(\mathrm{P}(c)) \subset \mathrm{P}(c)$ e $\mathrm{P}\left(P_{c}^{k}\right)=\mathrm{P}(c)$ para todo $k \geq 1 . \mathrm{P}(c)$ é o menor fachado que contém os pontos críticos de todos os iterados positivos de $P_{c}^{k}$ de $P_{c}$.

Definição 2.11. Quando $\mathrm{P}(c)$ for um conjunto finito denominamos $P_{c}$ de pós-crítico finito.

Introduziremos agora o Conjunto de Fatou.

Definição 2.12. O conjunto de Fatou de $P_{c}$ é por definição

$$
F(c):=\widehat{\mathbb{C}}-J(c)
$$

Seguem algumas propiedades de $F(c)$ mais outras de $J(c)$.

1. $F(c)$ é aberto, pois $J(c)$ é fechado;

2. $F(c)$ e $J(c)$ são totalmente invariantes;i.e., $P_{c}(F(c)) \subset F(c)$ e $P_{c}^{-1}(F(c)) \subset F(c)$

3. $F\left(P_{c}^{n}\right)=F(c) \Rightarrow J\left(P_{c}^{n}\right)=J(c)$

Para o caso de polinômios quadráticos (ou mesmo funções racionais em $\widehat{\mathbb{C}}$ ), a dinâmica das componentes do conjunto de Fatou é um problema completamente resolvido. Isto se resume nos seguintes dois teoremas devidos à Dennis Sullivan (1941).

Teorema 2.3 (Teorema das componentes não errantes de Sullivan). Toda componente do conjunto de Fatou é eventualmente periódica. Isto é, se $U$ é uma componente de $F(c)$, então, existe $(m, n) \in$ $\mathbb{N} \times(\mathbb{N}-\{0\})$ tal que $P_{c}^{n}\left(P_{c}^{m}(U)\right)=P_{c}^{m}(U)$. 
Teorema 2.4 (Teorema de classificação de Sullivan). Seja $f$ uma função racional com grau $(f) \geq 2$ e U uma componente periódica do conjunto de Fatou então, uma, e apenas uma, das seguintes opções é satisfeita por $U$

1. U é uma bacia atratora;

2. $U$ é uma pétala atratora de um cíclo parabólico,isto é, existe $z_{0} \in \partial U$ tal que todo ponto de $U$ converge para $z_{0}$ por iteração de $f$;

3. $U$ é simplesmente conexo e $\left.f\right|_{U}: U \longrightarrow U$ é conformemente conjugada a uma rotação irracional no disco unitário;

4. $U$ é duplamente conexo e $\left.f\right|_{U}: U \longrightarrow U$ é conformemente conjugada a uma rotação irracional em um anel(para uma definição de anel consulte 2.21).

Devido ao teorema do módulo máximo não temos a ocorrência do ítem 4 no caso polinomial.

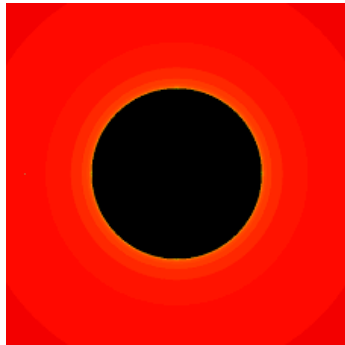

(a) bacia de atração

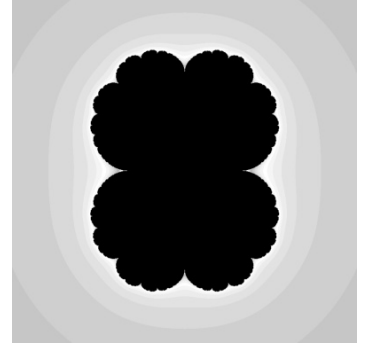

(b) Pétala atratora

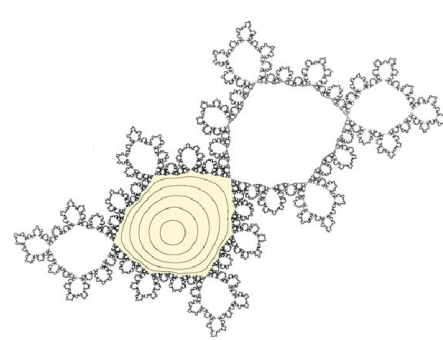

(c) Disco de Siegel

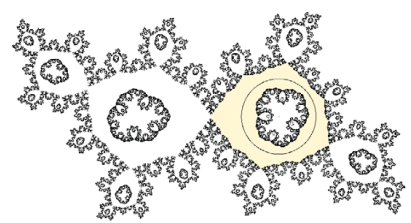

(d) Anel de Herman

Figura 2.4: Componentes do Fatou

Vimos na proposição 2.1 que o conjunto dos polinômios quadráticos definem uma família a um parâmetro denominada de família qudrática. Assim, em Dinâmica Holomorfa não estamos iteressados apenas no espaço de fase, dito comumente de espaço dinâmico, mas também no espaço de parâmetros $\mathbb{C}$.

\subsubsection{O conjunto de Mandelbrot}

$$
\begin{aligned}
\mathcal{M} & :=\left\{c \in \mathbb{C} ; J\left(P_{c}\right) \quad \text { é conexo }\right\} \\
& =\left\{c \in \mathbb{C} ; \sup _{n}\left\{\left|f_{c}^{n}(0)\right| ; n \in \mathbb{N}\right\}<\infty\right\}
\end{aligned}
$$

$\mathcal{M}$ tem sido de grande interresse devido sua beleza e por ser um objeto bastante complicado. É um exemplo de um compacto de $\mathbb{C}$ que possue uma topologia um tanto quanto intricada. Embora muito já se saiba sobre tal conjunto, algumas perguntas perduram irresolutas. $\mathcal{M}$ consiste em um exemplo de conjunto fractal com a propriedade de autossimilaridade de conter cópias de si, como é ilustrado na figura 2.6. Assim, podemos prosseguir fazendo tais ampliações em cada cópia de $\mathcal{M}$ obtida na ampliação anterior.

A aparição destas pequenas cópias de $\mathcal{M}$ próprio pode ser explicada a partir da teoria das aplicações do tipo polinômial desenvolvida por Douady e Hubbard. Uma das principais conjecturas em Dinâmica Holomorfa é sobre a topologia de $\mathcal{M}$, que contém um grande conteúdo dinâmico. 


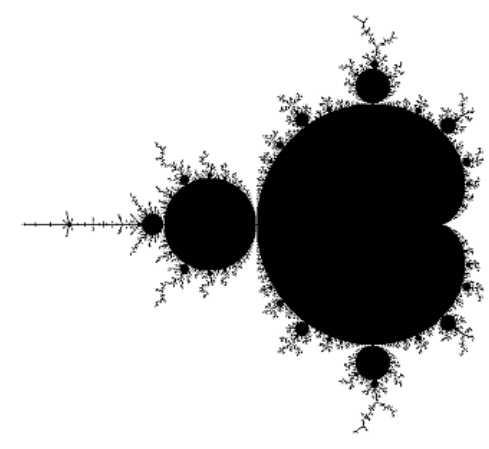

Figura 2.5: Conjunto de Mandelbrot

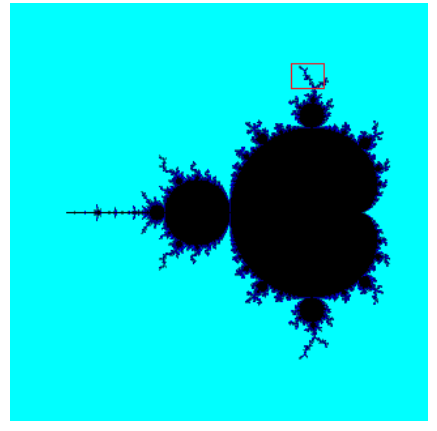

(a) Mandelbrot

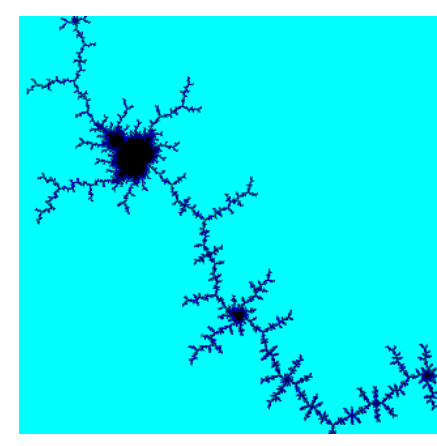

(b) pequena cópia de $\mathcal{M}$ em $\mathcal{M}$

Figura 2.6: Mandelbrot e um cópia sua. Zoom dado na região enquadrada na figura (a).

Conjectura 2.1.1 (Douady-Hubbard). $\mathcal{M}$ é localmente conexo.

Já temos pronto um modelo topológico-combinatório para $\mathcal{M}$, caso ele seja localmente conexo, devido à Douady que consiste no modelo dos discos apertados([Dou2]).

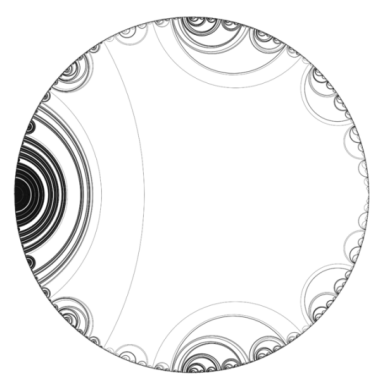

Figura 2.7: laminação geodésica do disco

\subsubsection{Ponto fixo}

Definição 2.13. $z_{0} \in \widehat{\mathbb{C}}$ é ponto fixo de $P_{c}$ se, e somente se, $P_{c}\left(z_{0}\right)=z_{0}$

Definição 2.14. O multiplicador de um ponto fixo $z_{0}$ é o número $\lambda:=P_{c}^{\prime}\left(z_{0}\right)$.

Os pontos fixos são classificados em relação ao seu multiplicador como segue:

1. Se $|\lambda|<1$ denominamos $z_{0}$ de ponto fixo atrator;

- Se $|\lambda|=0$ denominamos $z_{0}$ de ponto fixo super-atrator; 
2. Se $|\lambda|>1$ denominamos $z_{0}$ de ponto fixo repulsor;

3. Se $|\lambda|=1$ denominamos $z_{0}$ de ponto fixo indiferente:

- Se $\lambda=e^{2 \pi i \frac{p}{q}} \operatorname{com} \frac{p}{q} \in \mathbb{Q}$, denominamos $z_{0}$ de ponto fixo parabólico;

- Se não, denominamos $z_{0}$ de ponto fixo irracionalmente indiferente.

Isto posto, segue do que vimos na subseção 2.1.2 que o $\infty$ é ponto fixo super-atrator de qualquer polinômio quadrático $P_{c}$.

No estudo do comportamento local de uma aplicação holomorfa em um ponto fixo estamos interessados na existência de uma mudança de coordenadas canônica em uma vizinhança deste ponto. E decerto temos tais coordenadas.

Teorema 2.5 (Coordenada de Böttcher). Dado $P_{c}$. Para $R$ grande o suficiente existe uma única aplicação

$$
\phi:\{|z|>R\} \longrightarrow \mathbb{C}
$$

que é biholomorfa de $V:=\{|z|>R\}$ em $\phi(V)$ tal que $\phi\left(P_{c}(z)\right)=(\phi(z))^{2}$. $\phi$ é chamada de coordenada de Böttcher.

Teorema 2.6. Se $c \in \mathcal{M}$, a coordenada de Böttcher $\phi$ nos dá a uniformização de $\widehat{\mathbb{C}}-K\left(P_{c}\right)$, isto é, $\phi$ é um biholomorfismo aplicação de Riemann de $\widehat{\mathbb{C}}-K\left(P_{c}\right)$ até $\widehat{\mathbb{C}}-\overline{\mathbb{D}}$.

\subsubsection{Raios externos e equipotenciais}

Definição 2.15 (Raio externo). Dado $t \in \mathbb{R} \bmod 1$ definimos a curva $R_{t}:=\{z \in \mathbb{C} ; \arg (\phi(z))=$ t\} e denominamos de raio externo de $P_{c}$ com ângulo $t$.

Definição 2.16 (Equipotencial). Dado $R>1$ definimos a curva $E_{R}:=\{z \in \mathbb{C} ;|\phi(z)|=R\}$ e denominamos de equipotencial de $P_{c}$ com potêncial(altura) $\exp R$.

Como $P_{c}=\phi^{-1} \circ \theta \circ \phi$ em $\widehat{\mathbb{C}}-K\left(P_{c}\right)$, onde $\theta(z):=z^{2}$, temos $P_{c}\left(R_{t}\right)=R_{2 t}$ e $P_{c}\left(E_{R}\right)=E_{R^{2}}$. Por indução temos, $P_{c}^{n}\left(R_{t}\right)=R_{2^{n} t}$ e $P_{c}^{n}\left(E_{R}\right)=E_{R^{2 n}}$, sendo assim, os raios externos e as equipotencias invariantes pelo polinômio $P_{c}$. Em particular, se $t_{0}$ é periódico para $t \mapsto 2 t \bmod 1$, então, $R_{t_{0}}$ é aplicado sobre si por algum iterado de $P_{c}$. $R_{0}$ é o único raio de $K\left(P_{c}\right)$ que é fixo para $P_{c}$.

Definição 2.17. Diremos que um raio externo $R_{t}$ com $t \in \mathbb{R} \bmod 1$ é racional se $t$ for racional. $E$ diremos que $R_{t}$ é periódico se o ângulo $t$ for periódico para a aplicação $t \mapsto 2 t \bmod 1$.

Quando, para $t \in \mathbb{R} \bmod 1$ fixado, o limite $\lim _{r \searrow 1} \varphi^{-1}(r \exp (2 \pi i t))$ existir, o denotaremos por $\gamma(t)$ e diremos que o raio externo $R_{t}$ aterrissa no ponto $\gamma(t) \in \partial J(c)$. Diremos também que $\gamma(t) \in J(c)$ é um ponto de aterrissagem de $R_{t}$.

Listamos, a seguir, algumas propriedades dos raios externos. Para mais informações e resultados sobre este assunto consultem $§ 18-[M i l 1]$, Cap.6-[McM1].

Para o que segue assumamos $P_{c} \operatorname{com} c \in \mathcal{M}$.

Teorema 2.7. Se o raio $R_{t}$ aterrissa em um ponto $\gamma(t) \in J(c)$, então, o raio $R_{2 t}$ aterrissa no ponto $\gamma(2 t)=P_{c}(\gamma(t))$. Além do mais, cada raio da forma $R_{\frac{t+j}{2}}$ aterrissa em um dos pontos de $P_{c}^{-1}(\gamma(t))$ e cada ponto de $P_{c}^{-1}(\gamma(t))$ é ponto de aterrissagem de um destes raios. 

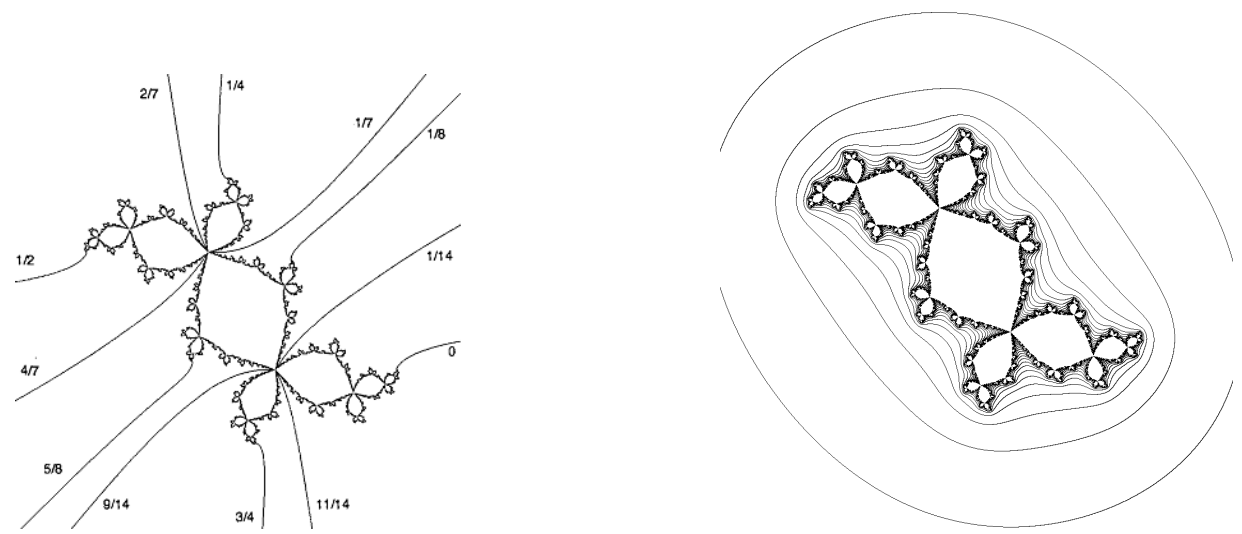

Figura 2.8: raios externos e equipotenciais

Note que se $R_{t}$ é periódico de período $p \geq 1$ e aterrissa em um ponto $\gamma(t)$, então $\gamma(t)$ é periódico com período $q$ que divide $p$.

Teorema 2.8 (Douady). Pontos períodicos repulsores e parabólicos são pontos de aterrissagem de pelo menos um raio externo periódico.

Teorema 2.9. Todo raio externo periódico aterrissa em um ponto do conjunto de Julia que é um ponto periódico repulsor ou parabólico. Caso o argumento do raio seja racional, mas não periódico(para a aplicação $t \mapsto 2 t)$, então, o raio aterrissa em um ponto eventualmente periódico não periódico.

Teorema 2.10. Se um raio aterrissa em $z_{0} \in J(c)$, então apenas um número finito de raios externos aterrissam neste ponto, e todos possuem o mesmo período.

Teorema 2.11. Seja $z_{0}$ um ponto parabólico ou repulsor para $P_{c}$. Então, o número de componebtes de $K(c)-\left\{z_{0}\right\}$ é igual ao número de raios externos que aterrissam em $z_{0}$.

Para uma prova destes teoremas consulte [Mil1],[McM1].

\subsubsection{Pontos fixos de $P_{c}$}

É certo, pelo teorema fundamental da álgebra, que cada polinômio $P_{c}$ tem dois pontos fixos. E com uma simples conta verificamos que existe um único parâmetro $c \in \mathbb{C}$ para o qual $P_{c}(z)-z$ tem raiz com multiplicidade 2 que, a saber, é $c=\frac{1}{4}$. Seguindo a notação posta por Douady-Hubbad denotaremos por $\beta$ o ponto fixo de $P_{c \neq 1 / 4}$ no qual o raio externo de argumento igual a 0 aterrissa. Ao outro ponto fixo denotaremos por $\alpha$. Geralmente estamos interessados em polinômios $P_{c}$ para os quais os pontos fixos são repulsores.

Teorema 2.12. O ponto fixo $\beta$ não separa o Julia cheio $K_{c}$, isto é, $K_{c}-\beta$ possuem apenas uma componente conexa.

Corolário 2.1. Um ponto fixo repulsor ou parabólico $z_{0}$ separa o Julia cheio $K_{c}$ se, e somente se, $z_{0}=\alpha$. 


\subsubsection{O quebra-cabeça de Yoccoz.}

A partir do sistema de curvas invariantes por $P_{c}$ formado pelos raios externos e as equipotenciais construímos uma partição dinâmica de $\widehat{\mathbb{C}}$ globalmente invariante que será uma ferramenta combinatória de fundamental importância para o estudo da dinâmica de $P_{c}$. A esta partição chamamos de Quebra-cabeça de Yoccoz.

Consideremos $P_{c}$ com $c \in \mathcal{M}$ e cujos pontos fixos sejam repulsores. Por simplicidade suporemos ainda que a órbita positiva do ponto crítico $z=0$ seja disjunta do ponto fixo $\alpha$. Assim, estaremos em codições de posteriormente definir uma outra ferramenta combinatória denominada tabela crítica e evitamos o caso em que o conjunto pós-critico é finito cuja dinâmica já se encontra totalmente entendida(por métodos que não involvem o quebra-cabeça de Yoccoz).

Seja $\varphi$ a aplicação de Riemann de $\widehat{\mathbb{C}}-\mathbb{D}$ para $\widehat{\mathbb{C}}-K(c)$ normalizada por $\varphi^{\prime}(\infty)=1$. Consideremos o domínio $D$ contendo $K(c)$ cuja fronteira é a imagem por $\varphi^{-1}$ da círculo centrado na origem com raio igual a 2. Este disco topológico fica dividido em $q$ subdomínios em que $q>1$ é o número de raios externos que aterrissam no ponto fixo $\alpha$. Chamaremos tais domínios de peças e as denotaremos por $Y_{0}^{i}$ em que $P_{c}^{i}(0) \in Y_{0}^{i}$,para $i=0,1, \cdots, q-1$. Tais peças, $\left\{Y_{0}^{i}\right\}_{i=0}^{q-1}$, formam o nível zero do quebra-cabeça de Yoccoz, $Y_{0}=\cup_{i=0}^{q-1} Y_{0}^{i}$, que nada mais é do que o domínio $D$ com os seguimentos de raios externos que aterrissam em $\alpha$ destacados.

Cada peça $Y_{0}^{i}$ é um disco fechado cuja fronteira consiste na união do ponto fixo $\alpha$, dois arcos de raios externos e um arco da equipotencial $\varphi^{-1}(\partial B(0,2))=\partial D$.

Cada peça do nível $n+1$ fica contida dentro de uma única peça do nível $n$, pois os raios que aterrissam em $J(c)$ no nível $n+1$ diferentes dos raios do nível anterior $n$ aterrissam em pontos de $J(c)$ contidos em alguma peça, mas, os raios não se intersectam pois $\varphi$ é injetora.

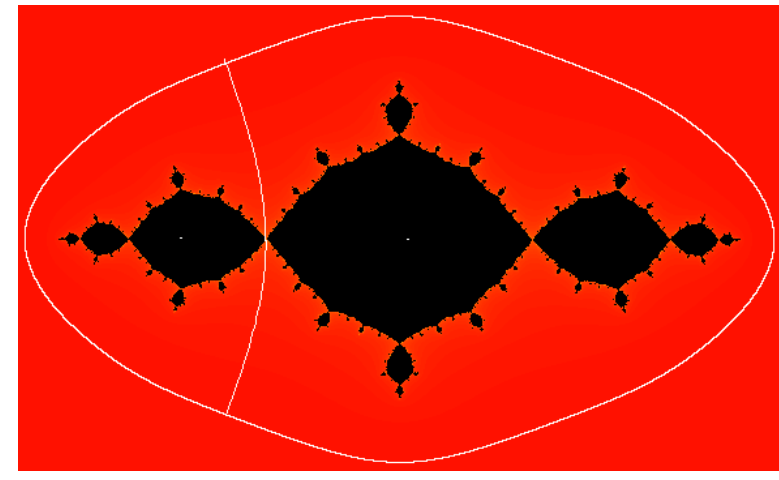

(a) nível 0

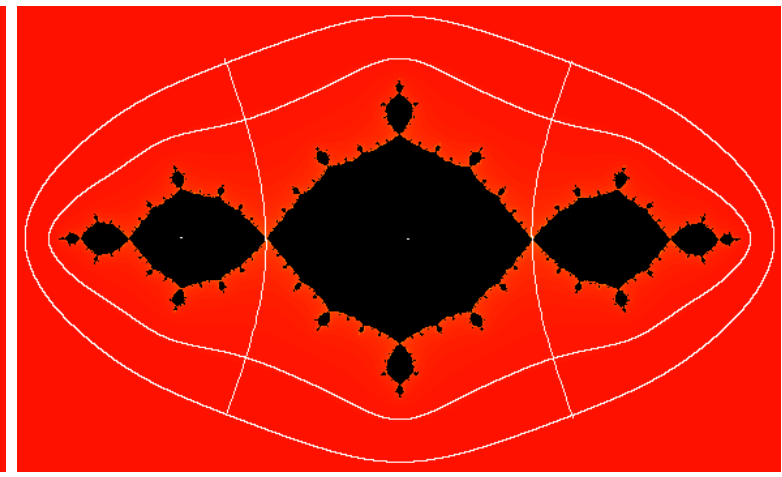

(b) nível 1

Figura 2.9: Quebra-cabeça de Yoccoz da basílica

As peças do quebra-cabeça de Yoccoz do nível $n+1$ são definidas indutivamente como sendo as componentes de $P_{c}^{-1}\left(Y_{n}^{i}\right)$ na qual $Y_{n}^{i}$ é uma peça do nível $n$. Uma peça de um nível $n$ será, evidentemete, denotada por $Y_{n}^{i}$ para algum $i=0,1, \cdots, r_{n}$ em que $r_{n}$ é o número de raios externos no nível $n$. As peças do nível $n$ possuem seus interiores disjuntos e cobrem todo o conjunto de Julia de $P_{c}$.

Note que quando tomamos a pré-imagem do nível zero $Y_{0}$ do quebra-cabeça obtemos o domínio $P_{c}^{-1}(D)$ que contém $J(c)$ mas, que contém seguimentos das pré-imagens dos raios que aterrissam no ponto fixo $\alpha$, que pelo teorema 2.7, também são raios externos de $K(c)$. Assim, à medida que cresce o nível, o quebra-cabeça fica mais fino(como uma partição dinâmica do Julia cheio). Para 
denotar uma peça do nível $n$ do quebra-cabeça que contém um ponto $z \in \widehat{\mathbb{C}}$ usaremos a notação, $Y_{n}(z)$.

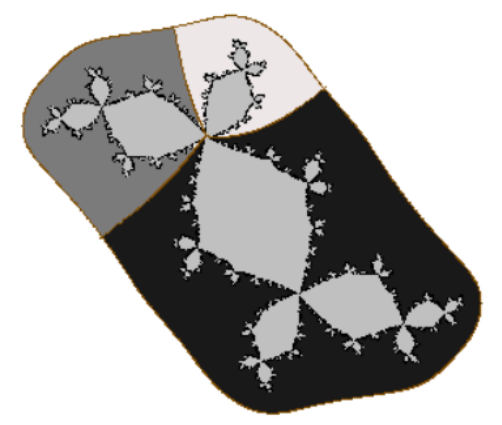

Figura 2.10: Quebra-cabeça do Coelho de Douady-nível 0

Na figura 2.10 uma peça cinza vai até aquela pintada com um tom um pouco mais escuro, e no final a peça mais escura vai sobre a mais clara como um recobrimento duplo.
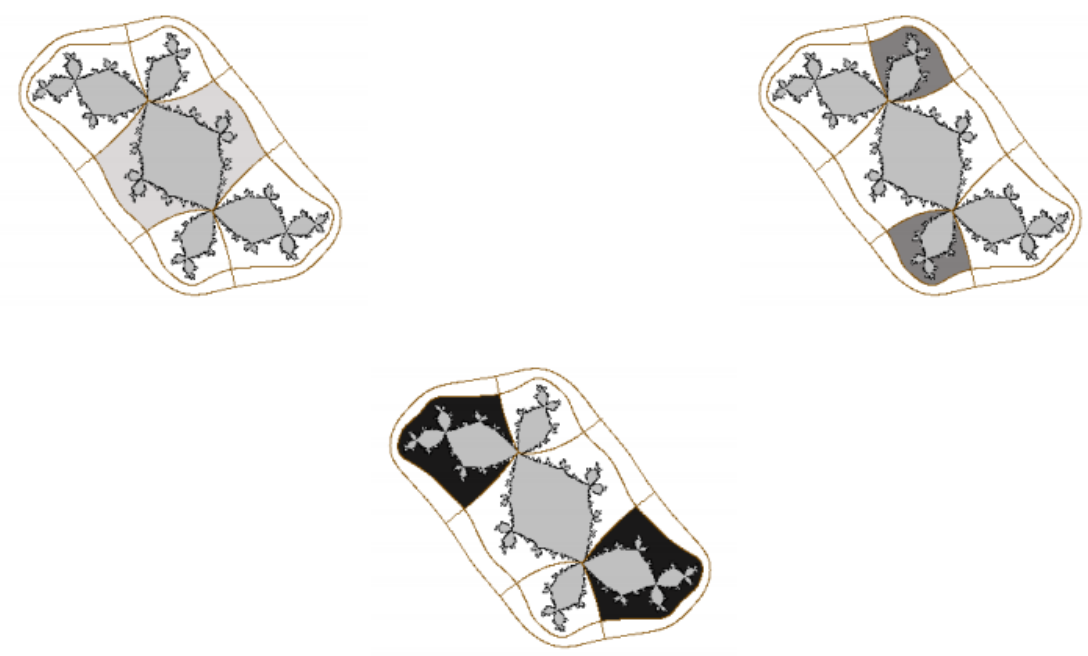

Figura 2.11: Quebra-cabeça do Coelho de Douady-nível 1

Na figura 2.11 temos o nível 1 do quebra-cabeça as peças pintadas são pré-imagens daquelas do nível 0 com o mesmo tom de cinza.

Definição 2.18 (Grande órbita). Dado $z_{0} \in \widehat{\mathbb{C}}$ e $P_{c}$. A grande órbita de $z_{0}$ por $P_{c}$ é o conjunto $G O\left(z_{0}, P_{c}\right):=G O\left(z_{0}\right):=\left\{w \in \widehat{\mathbb{C}} ; \exists(k, l) \in \mathbb{N}^{2}\right.$ tal que $\left.P_{c}^{k}(w)=P_{c}^{l}\left(z_{0}\right)\right\}$

Decerto, segue do teorema 2.7 que cada ponto da grande órbita de $\alpha$ é ponto de aterrissagem de um raio externo de $K(c)$. Deste modo, se $z_{0} \in K(c)$ não pertence a grande órbita de $\alpha$, então para cada nível $n \in \geq 0$ existe uma única peça $Y_{n}^{i}$ a qual $z_{0}$ pertence. Note ainda, que da definição indutiva das peças do nível $n+1$ decorre, $Y_{n}(f(z))=P_{c}\left(Y_{n+1}(z)\right)$.

\subsection{Aplicação do tipo polinomial}

Assim como a nomeclatura indica, aplicações quaseconformes são aplicações entre domínios da esfera de Riemann que localmente se comportam com um polinômio. 
Toda aplicação holomorfa de $\widehat{\mathbb{C}}$ em $\widehat{\mathbb{C}}$ Aplicações racionais, isto é, aplicações que são o quociente entre dois polinômios, podem ter restrições a subconjuntos de $\widehat{\mathbb{C}}$ onde se comportam como polinômios de grau menor do que o seu. Isto occorre, por exemplo, na vizinhança de um ponto fixo atrator geométrico, onde se comporta como uma polinômio de grau igual a 1.

Definição 2.19 (Aplicação do tipo polinomial-Douady-Hubbard). Uma aplicação do tipo polinomial de grau d é um terno $(f, U, V)$ no qual $f: U \longrightarrow V$ é uma aplicação holomorfa própria de grau d com $U$ e $V$ conformemente isomorfos ao disco unitário $\mathbb{D}$ e $\bar{U} \subset V$.

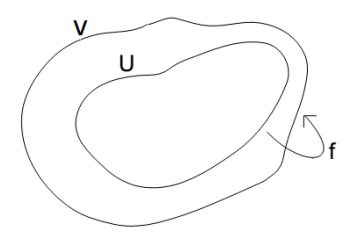

Figura 2.12: aplicação do tipo polinômial

Segue da fórmula de Riemann-Hurwitz(veja[Mil1]pag.72) que $(f, U, V)$ possuem $d-1$ pontos críticos em $U$ contados com multiplicidade. Em particular, no caso em que $d=2$ temos um único ponto crítico.

Exemplo 2.2. Considere $P_{c}$. Então, $\left(P_{c}, Y_{1}, Y_{0}\right)$ é uma aplicação do tipo polinômial de grau 2. Costuma-se chamar uma aplicação do tipo polinomial de grau 2 de aplicação do tipo quadrático.

Definição 2.20. Seja $(f, U, V)$ uma aplicação do tipo polinomial. Em analogia aos polinômios quadráticos, definimos os seguintes conjuntos

1. O Julia cheio:

$$
K(f):=\bigcap_{n=1}^{\infty} f^{-n}(V)
$$

isto é, $K(f)$ é o conjuntos dos pontos de $U$ que não escapam de $U$ por interação de $f$;

2. Conjunto de Julia:

$$
J(f):=\partial K(f)
$$

\section{Conjunto Pós-crítico:}

$$
\mathrm{P}(f):=\bigcup_{c \in C(f)} \overline{\bigcup_{n=1}^{\infty} f^{n}(c)},
$$

em que $C(f)$ é o conjunto dos pontos críticos de $(f, U, V)$.

Sabe-se que $K(f)$ é compacto e quando é conexo é também simplesmente conexo. Assim como para as aplicações polinomias temos o seguinte resultado.

Teorema 2.13. $K(f)$ é conexo se, e somente se, todos os pontos críticos de $(f, U, V)$ pertencem ao $K(f)$. E se nenhum ponto crítico de $(f, U, V)$ pertence ao $K(f)$, então, $K(f)$ é um conjunto de Cantor. 


\subsection{Aplicação quaseconforme.}

Aplicações quaseconformes formam um ramo de estudo em Análise Complexa. Para John Hamal Hubbard estas aplicações são, de algum modo, mágicas, com propriedades que parecem contraditórias. São suaves o suficiente de modo que muitos cálculos ainda valham, como: a regra da cadeia e regras integrais para comprimentos e áreas. E, no entanto, são rígidas o suficiente para mudar o valor da derivada de um ponto fixo por conjugação(vide [Hu]).

Existem várias definições equivalentes para aplicaçôes quaseconformes. Apresentamos a seguir uma definição analítica, mas para tanto será necessário darmos antes algumas definições.

Definição 2.21 (Função teste). Fixado um aberto $U \subset \mathbb{C}$, uma função teste em $U$ é uma função $h: U \longrightarrow \mathbb{C}$ de classe $\mathrm{C}^{\infty}$ com suporte compacto em $U$, isto é, que é nula fora de um subconjunto compacto de $U$. Denotamos o cunjunto das funções teste em $U$ por $\mathrm{C}_{c}^{\infty}(U)$.

Com uma topologia adequada, $\mathrm{C}_{c}^{\infty}(U)$ é um espaço vetorial topológico de dimensão infinita.

Definição 2.22 (Distribuicão). Uma distribuição em $U$ é uma funcional linear contínuo $T$ : $C_{c}^{\infty}(U) \longrightarrow \mathbb{C}$.

Definição 2.23. Para $f \in \mathrm{C}^{1}(U)$, definimos

$$
\begin{aligned}
\partial f & :=\frac{1}{2}\left(\frac{\partial f}{\partial x}-i \frac{\partial f}{\partial y}\right) \\
\bar{\partial} f & :=\frac{1}{2}\left(\frac{\partial f}{\partial x}+i \frac{\partial f}{\partial y}\right)
\end{aligned}
$$

Definição 2.24 (Derivadas distribucionais.). Uma função $f: U \longrightarrow \mathbb{C}$ em $L_{\text {loc }}^{2}(U)$ tem derivadas distribucionais em $L_{\text {loc }}^{2}(U)$ se existem funçôes $f_{1}, f_{2}: U \longrightarrow \mathbb{C}$ em $L_{\text {loc }}^{2}(U)$ tais que para qualquer $h \in C_{c}^{\infty}(U)$ valha

$$
\begin{aligned}
\int_{U} f_{1} \cdot h d m & =-\int_{U} f_{1} \cdot \partial h d m \\
\int_{U} f_{2} \cdot h d m & =-\int_{U} f_{2} \cdot \bar{\partial} h d m
\end{aligned}
$$

É comum denotar, $f_{1}=\partial f$ e $f_{2}=\bar{\partial} f$. Isto se justifica com o fato de que quando $f \in C^{1}(U), f_{1}$ e $f_{2}$ coincidirem com as usuais derivadas parciais $\partial f$ e $\bar{\partial} f$.

Definição 2.25 (aplicação quaseconforme-definição analítica). Sejam $U$ e $V$ subconjuntos abertos de $\mathbb{C}$ e $k:=\frac{K-1}{K+1}$, tal que $0 \leq k<0$. Uma aplicação $f: U \longrightarrow V$ é $K$-quaseconforme se:

1. f é um homeomorfismo;

2. as derivadas parciais distribuicionais de $f$ estão em $L_{l o c}^{2}(\mu)$, e satisfaz

$$
|\partial f| \leq k|\bar{\partial} f|
$$

em $L_{l o c}^{2}(\mu)$ 
chamaremos de constante de quaseconformalidade de $f$ ao menor $K$ para o qual valha a definição dada acima.

Podemos entender a constante $K$ como uma medida de o quanto $f$ está próxima de ser conforme. O seguinte teorema indica isso.

Teorema 2.14. $f: U \longrightarrow V$ é 1-quaseconforme se, e somente se, é conforme.

Melhor entendemos a definição dada quando supomos $f$ de classe $\mathrm{C}^{1}(U)$.

Toda aplicação $\mathbb{R}$-linear $T: \mathbb{C} \longrightarrow \mathbb{C}$ pode ser apresentada da seguinte forma,

$$
T(z)=a z+b \bar{z}
$$

Pode-se verificar que a pré-imagem do circulo unitário por $T$ é uma elipse centrada na origem com semieixo maior medindo $\frac{1}{|a|-|b|}$, semieixo menor medindo $\frac{1}{|a|+|b|}$ com argumento igual a $\frac{\arg (b)-\arg (a)}{2}$. Disto decorre que $\operatorname{det}(T)=|a|^{2}-|b|^{2}$ e $\|T\|=|a|+|b|$.

Para $f \in \mathrm{C}^{1}(U)$ e $z_{0} \in U, D f_{z_{0}}: T_{z_{0}} U \longrightarrow T_{f\left(z_{0}\right)} U$ é uma aplicação $\mathbb{R}$-linear. E neste caso, temos

$$
\begin{aligned}
{\left[D f_{z_{0}}\right](u) } & =\partial f\left(z_{0}\right) u+i \bar{\partial} f\left(z_{0}\right) \bar{u} \mathrm{com} \\
\left\|\left[D f_{z_{0}}\right]\right\| & =\left|\partial f\left(z_{0}\right)\right|+\left|\bar{\partial} f\left(z_{0}\right)\right| \\
\operatorname{det}\left(\left[D f_{z_{0}}\right]\right) & =\left|\partial f\left(z_{0}\right)\right|^{2}-\left|\bar{\partial} f\left(z_{0}\right)\right|^{2}
\end{aligned}
$$

Note que

$$
\frac{|a|+|b|}{|a|-|b|}=\frac{1+\frac{|b|}{|a|}}{1-\frac{|b|}{|a|}} \leq \frac{1+k}{1-k}=K
$$

e que a desigualdade 2.14 implica que $f$ preserva orientação.

Ou seja, uma aplicação $K$-quaseconforme de classe $C^{1}$ é um difeomorfismo que presenva orientação e cuja derivada mapeia discos infinitesimais em elipses infinitesimais com excentricidade no máximo $K$.

Definição 2.26 (Coeficiente de Beltrami). À função mensurável $\mu(z):=\frac{\bar{\partial} f}{\partial f}(z)=\frac{\bar{\partial} f(z)}{\partial f(z)}$ é denominada coeficiente de Beltrami, ou ainda, dilatação complexa de $f$.

As aplicações quaseconformes são de fundamental importância na Dinâmica Holomorfa em geral. E a inserção desta tão frutífera ferramenta nesta área se deve ao seguinte celebrado teorema conhecido como Teorema de Ahlfors-Bers ou Teorema mensurável da aplicação de Riemann.

Teorema 2.15. Para qualquer função mensurável $\mu: \widehat{\mathbb{C}} \longrightarrow \widehat{\mathbb{C}}$ com $\|\mu\|_{\infty} \leq 1$, existe uma única aplicação quaseconforme $\varphi_{\mu}: \widehat{\mathbb{C}} \longrightarrow \widehat{\mathbb{C}}$ cujo coeficiente de Beltrami(dilatação complexa) é $\mu$ e que mantém fixados os pontos $0,1, \infty$.

Voltamos a tratar das aplicações do tipo polinômial. A partir das aplicações quaseconformes podemos introduzir duas novas noções de equivalência entre sistemas dinâmicos. 
Definição 2.27. (equivalência quaseconforme e híbrida)

1. Dois polinômios quadráticos $P_{a}$ e $P_{b}$ são quaseconformemente equivalentes se existe uma aplicação quaseconforme $\varphi: U \longrightarrow U^{\prime}$ entre as vizinhanças $U$ e $U^{\prime}$ de $K(a)$ e $K(b)$ que conjuga $P_{a}$ ao $P_{b}$. A aplicação $\varphi$ é chamada de conjugação quaseconforme.;

2. Dois polinômios quadráticos $P_{a}$ e $P_{b}$ são hibridamemente equivalentes se são quaseconformemente equivalentes e a conjugação quaseconforme $\varphi$ satisfaz $\bar{\partial} \varphi=0$ em $K(a)$.

O seguinte importantíssimo resultado devido ao A. Douady e J.H. Hubbard justifica a denominação "aplicação do tipo polinômial".

Teorema 2.16 (Teorema de retificação-Douady-Hubbard). Toda aplicação do tipo polinomial $(f, U, V)$ de grau d é hibridamente equivalente a um polinômio $P$ de grau d. Além disto, se $K(f)$ for conexo, então, o polinômio $P$ é único a menos de uma conjugação por uma aplicação afim.

No caso quadrático este teorema toma a seguinte forma

Teorema 2.17 (Teorema de retificação-caso quadrático). Toda aplicação do tipo quadrático $(f, U, V)$ é hibridamente equivalente a um polinômio $P_{c}$. Além disto, se $K(c)$ for conexo, então $P_{c}$ é único.

\subsection{Hiperbolicidade e Rigidez quaseconforme}

No que segue nos restringiremos a discutir a hiperbolicidade apenas para a família quadrática. Mais informações sobre este conteúdo podem ser encontrados em [Mil1],[MSS],[CG].

Existe uma classe de polinômios quadráticos muito especial que apresenta uma dinâmica relativamente simples e bem compreendida. Tais aplicações são denominadas hiperbólicas. Elas detém uma das mais importantes questões em aberto em Dinâmica Holomorfa, certamente a mais antiga, que foi posta por Pierre Fatou.

Conjectura 2.4.1 (Densidade de hiperbolicidade-caso quadrático). Os polinômios quadráticos hiperbólicos formam um subconjunto aberto e denso no espaço dos polinômios quadráticos.

A qualidade principal desses sistemas dinâmicos é preservar a topologia dos principais conjuntos invariantes, sob pequenas variações dos parâmetros.

De maneira mais formal, que significa "ser hiperbólico" para um polinômio quadrático?

Definição 2.28. Um polinômio quadrático $P_{c}$ é hiperbólico se sua órbita crítica tende para um cíclo atrator. Diz-se também que o parâmetro c é hiperbólico.

Assim, é evidente que se $c \notin \mathcal{M}$, então, $P_{c}$ é hiperbólico. Pois, neste caso, sabemos que $P_{c}^{n}(0) \rightarrow$ $\infty$ e $\infty$ é ponto fixo super-atrator. Deste modo, podemos redefinir a hiperbolicidade dos polinômios quadráticos como:

Definição 2.29. Um polinômio quadrático é hiperbólico se:

1. $c \notin \mathcal{M}$, ou

2. $c \in \mathcal{M}$ e $P_{c}$ possue um cíclo atrator em $\mathbb{C}$. 
Por exemplo, o polinômio $z \mapsto z^{2}$ tem a origem como ponto fixo atrator. Para $c$ suficientemente pequeno (exemplo: $c=0,1), z \mapsto z^{2}+c$ também tem um ponto fixo atrator.

Para entender de maneira global a dinâmica dos polinômios quadráticos, é importante juntar os parâmetros produzindo sistemas dinâmicos "semelhante", por exemplo, todos os polinômios com um ponto fixo que seja atrator.

Definição 2.30 (Componente hiperbólica de $\mathcal{M}$ ). Uma componente do interior de $\mathcal{M}$ é hiperbólica se contiver um parâmetro hiperbólico. Uma componente do interior de $\mathcal{M}$ que não é hiperbólica é denominada componente estranha.

A componente hiperbólica contendo o paramêtro hiperbólico $c=0$ é o interior de uma cardioide no conjunto de Mandelbrot. O fato de ser um polinômio hiperbólico tem muitas consequências: o conjunto de Julia de qualquer polinômio quadrático hiperbólico tem área zero e quando é conexo é, também, localmente conexo; cada órbita é repulsora ou atratora. Todos os polinômios quadráticos próximos de um polinômios quadrático hiperbólico também são hiperbólicos.

Na verdade, Mañé, Sad e Sullivan em [MSS] mostraram que, para um parâmetro hiperbólico, $J(c)$ se move continuamente(na topologia de Hausdorff) quando deformamos $P_{c}$ ao longo de polinômios quadráticos hiperbólicos. Assim, no caso $c=0$ o conjunto de Julia é um círculo, e para $c$ dentro da componente de $c=0$ (a famosa cardioide do conjunto de Mandelbrot) a topologia de $J(c)$ fica constante, isto é, $J(c)$ é um círculo topológico.

Mas podemos dizer muito mais sobre essa permanência das principais propriedades de um sistema dinâmico ao redor de um sistema hiperbólico. Já vimos que a topologia do conjunto de Julia fica a mesma. Mas, podemos perguntar se a dinâmica fica a mesma, isto é, se tem uma conjugação topológica da dinâmica com a dinâmica do parâmetro hiperbólico.

Definição 2.31 (estabilidade estrutural). Uma aplicação racional $R$ de grau dé estruturalmente estável se existe uma vizinhança de $R$ no espaço das aplicações racionais com o mesmo grau tal que nesta vizinhança toda aplicação seja topologicamente conjugada à $R$.

Pedir uma conjugação topológica global seria muito exigente, mas podemos pedir uma conjugação das dinâmicas restritas nos conjuntos de Julia, o que parece mais razoável. Por isso, Mañé, Sad e Sullivan[MSS] introduziram a noção de aplicações racionais $J$-estáveis. A relação com a hiperbolicidade é muito forte. É conjecturado que toda aplicação $J$-estável na família de todas as aplicações racionais com grau $d$ fixado é hiperbólica[McM1].

Definição 2.32 (J-estabilidade). Um polinômio $P_{c}$ é J-estável se para alguma vizinhança sua no espaço dos polinômios quadrático todos os polinômios aí contidos são topologicamente conjugados $\grave{a} P_{c}$ sobre o seus conjuntos de Julia e $J(x)$ depende continuamente na topologia de Hausdorff do parâmetro $x$ nesta vizinhança. Isto é, para cada $P_{a}$ em uma vizinhança de $P_{c}$ existe um homeomorfismo $h: U \longrightarrow U^{\prime}$ em que $U$ e $U^{\prime}$ são vizinhanças de $J(c)$ e $J(a)$, respectivamente, tal que $P_{c}=h^{-1} \circ P_{a} \circ h$ em $J(c)$. Diz-se que a familia quadrática é J-estável em c para um tal parâmetro.

Conjectura 2.4.2 (Caso quadrático). Todo polinômio quadrático J-estável é hiperbólico.

Na dinâmica holomorfa é de grande interesse se saber isso, pois temos o seguinte resultado:

Teorema 2.18 (Mañé, Sad e Sullivan[MSS]-caso quadrático). O conjunto dos parâmetros Jestáveis $\mathcal{M}^{e s t .}:=\left\{c \in \mathcal{M} ; P_{c}\right.$ é J-estável $\}$ é um subconjunto aberto e denso de $\mathcal{M}$. 
A ferramenta principal no estudo da deformação dos conjuntos de Julia (com a variação do parâmetro) é a noção chave de movimento holomorfo estudada por Mañé, Sad e Sullivan em [MSS].

Um movimento holomorfo é basicamente uma família de injeções parametrizada por um complexo, com boas propriedades de regularidade.

Definição 2.33 (Movimento Holomorfo). Seja $\Omega$ um subconjunto de $\widehat{\mathbb{C}}$. Um movimento holomorfo de $\Omega$ parametrizado pelo disco unitário $\mathbb{D}$ é uma aplicação $\Phi: \mathbb{D} \times \Omega \longrightarrow \widehat{\mathbb{C}}$ tal que

1. Para cada $z \in \Omega$, a aplicação $\lambda \longmapsto \Phi(\lambda, z)$ é holomorfa em $\mathbb{D}$;

2. Para cada $\lambda \in \mathbb{D}$, a aplicação $z \longmapsto \Phi(\lambda, z)$ é injetora;

3. $E \Phi(0, \cdot)=$ Id.

Um exemplo simples é o movimento de um ponto fixo atrator, com a variação do parâmetro. Um exemplo mais complicado é o movimento do círculo topológico $J(c)$ quando $c$ é perto de $c=0$. Esses movimentos holomorfos têm a propriedade surpreendente de ter uma extensão automática à toda esfera de Riemann:

Definição 2.34 (O $\lambda$-lema [MSS], [S1], [McM1]). Se $\Phi: \mathbb{D} \times \Omega \longrightarrow \widehat{\mathbb{C}}$ é um movimento holomorfo, então,

1. admite uma extenção a um movimento holomorfo de $\widehat{\mathbb{C}}$ parametrizado por $\mathbb{D}$;

2. E para cada disco $D(0, r)$ com $0<r<1$ a extensão $\Phi_{\lambda}(z): \widehat{\mathbb{C}} \longrightarrow \widehat{\mathbb{C}}$ é $K(r)$-quaseconforme $\operatorname{com} K(r)=\frac{1+r}{1-r}$.

Em particular, note que a aplicação $\Phi: \mathbb{D} \times \bar{\Omega} \longrightarrow \widehat{\mathbb{C}}$ é continua.

Pode-se encontrar uma outra definição de movimento holomorfo e algumas versões do $\lambda$-lema em que toma-se uma variedade complexa(conexa) $M$ em vez do disco unitário $\mathbb{D}$ como o espaço de parâmetro.

Voltando ao assunto da hiperbolicidade, existe mais uma caracterização dos parâmetros hiperbólicos utilizando a noção de campo de linhas invariante. Essa definição é um pouco mais técnica, mas é bem útil para a classificação dos sistemas dinâmicos.

Definição 2.35 (Campo de linhas invariante(univalente)).

- Seja $f$ uma aplicação holomorfa em $\widehat{\mathbb{C}}$. Um campo de linhas invariante por $f$ suportado num subconjunto mensurável $\Omega \subset \widehat{\mathbb{C}}$ é uma família de pares $(z, L(z))$ nos quais $z \in \Omega$ e $L(z)$ é um $\mathbb{R}$-subspaço de dimenção 1 de $T_{z} \widehat{\mathbb{C}}$, tal que

1. $m(\Omega) \neq 0$;

2. $f^{-1}(\Omega)=\Omega$;

3. a inclinação de $L(z)$ varia mensuravelmente em $\Omega$;

4. $f^{\prime}(L(z))=L(f(z))$.

- Um campo de linhas $\mu$ é univalente em um domínio $\Omega$ se for o pullback do campo de linhas horizontais por uma aplicação univalente em $\Omega$. Isto é, se $\mu=h^{*}(d \bar{z} / d z)$. 
A relação com a hiperbolicidade é imediata:

Teorema 2.19 (Campo de linhas invariante e hiperbolicidade). Um ponto $c \in \mathcal{M}$ pertence a uma componente não hiperbólica de $\mathcal{M}$ se, e somente se, $J(c)$ tem medida positiva e suporta um campo de linhas invariante.

Utilizando essa noção, podemos reformular a questão da densidade da hiperbolicidade.

Corolário 2.2. A dinâmica hiperbólica é densa na Família Quadrática se, e somente se, nenhum polinômio quadrático suporta em seu conjunto de Julia um campo de linhas invariante.

Já sabemos que os parâmetros dentro da mesma componente hiperbólica produzem sistemas dinâmicos bem semelhantes (pelo menos do ponto de vista topológico). Agora, que podemos dizer sobre a classificação quaseconforme dos polinômios quadráticos?

A resposta definitiva é a seguinte:

Teorema 2.20 (Classificação quaseconforme dos Polinômios Quadráticos). Uma classe de equivalência quaseconforme de polinômios quadráticos é de uma das seguintes formas:

1. o complementar do conjunto de Mandelbrot;

2. é uma componente hiperbólica de $\mathcal{M}$ menos o seu centro;

3. é o centro de uma componente hiperbólica;

4. uma componente estranha do interior de $\mathcal{M}$;

5. um único ponto da fronteira de $\mathcal{M}$.

$\mathrm{Na}$ classificação acima, deveríamos observar que conjeturalmente as componentes estranhas não existam. Dentro da lista das possibilidades, os itens 3 e 5 são diferentes.

Definição 2.36. Diremos que uma classe de equivalência para uma relação de equivalência definida em $\mathbb{C}$ é rígida se ela for igual a um único ponto $c \in \mathbb{C}$.

Assim, com esta notação, as classes quaseconformes dos itens 3 e 5 são rígidas.

Definição 2.37 (Rigidez quaseconforme). $P_{c}$ é quaseconformemente rígido se todo polinômio $P_{b}$ quaseconformemente equivalente a $P_{c}$ for, na verdade, conformemente equivalente a $P_{c}$.

Conjectura 2.4.3 (Rigidez quaseconforme). A classe de equivalência quaseconforme e conforme de um polinômio quadrático não hiperbólico $P_{c}$ é formada apenas pelo próprio polinômio.

Para ilustrar as várias definições dadas, vamos estudar a relação entre rigidez e existência de campos de linhas invariantes.

Teorema 2.21. Rigidez quaseconforme é equivalente a não existência de polinômios uadráticos que suportam em seu Julia um campo de linhas invariante.

Demonstração. Decerto, temos tal equivalência, pois, pelo teorema 2.20, a rigidez quaseconforme implica a não existência de componentes estranhas do interior de $\mathcal{M}$. Logo pelo teorema 2.19 nenhum polinômio quadrático suporta em seu Julia um campo de linhas invariante. 
Por outro lado, se supomos que nenhum polinômio quadrático suporta em seu Julia um campo de linhas invariante, então, não existem componentes estranhas do interior de $\mathcal{M}$ em razão do teorema 2.19. Logo, as únicas classes quaseconformes rígidas são os centro de componentes hiperbólicas e pontos da fronteira de $\mathcal{M}$. Portanto, todo parâmetro não hiperbólico é quaseconformemente rígido.

Em conclusão, podemos entender um pouco melhor a organização global da dinâmica quadrática. Sem duvidas, o conjunto dos parâmetros (i.e., o conjunto de Mandelbrot) tem uma topologia extremamente complicada, mas a informação dentro dele é claramente organizada. Grandes regiões do conjunto de Mandelbrot (as componentes hiperbólicas) juntam parâmetros produzindo sistemas dinâmicos semelhantes (do ponto de vista topológico, quaseconforme e conforme). Cada componente hiperbólica contém um parâmetro especial no seu centro, com uma propriedade de rigidez. Finalmente, a reunião dessas componentes, conjeturalmente, produz o interior do conjunto de Mandelbrot.

\subsection{Definições e resultados gerais}

Dentro do contexto geométrico um objeto que consideraremos muitas vezes é o seguinte:

Definição 2.38. Um anel é qualquer superfície de Riemann A que seja conformemente isomorfa a um anel canônico $A(R):=\{z \in \mathbb{C} ; 1<|z|<R\}$ ou a $\mathbb{D}^{*}:=\{z \in \mathbb{C} ; 0<|z|<1\}$ ou a $\mathbb{C}^{*}=\mathbb{C}-\{0\}$

Particularmente, qualquer subconjunto aberto de $\widehat{\mathbb{C}}$ cujo complementar é formado por duas componentes conexas na qual uma delas é um subconjunto compacto de $\mathbb{C}$ é um anel.

Quando $A$ é isomorfa a um anel canônico $A(R) ;=\{z \in \mathbb{C} ; 1<|z|<R\}$ temos um número real positivo associado que contém informação sobre a sua geometria.

Definição 2.39. O módulo de um anel $A$ conformente isomorfo a $A(R)$ é o número

$$
\bmod (A):=\frac{\log (R)}{2 \pi}
$$

Definimos ainda, $\bmod (A)=+\infty$ quando $A$ é conformemente isomorfo a $\mathbb{D}^{*}$ ou a $\mathbb{C}^{*}$.

O módulo é um invariante conforme(problema 2-f[Mil1]).

Dado um disco topológico $D$ em $\mathbb{C}$ e $K$ com fecho compacto em $D$, definimos o seguinte número que nos dá uma medida geométrica da quantidade do espaço em $D$ ao redor de $K \subset D$ :

$$
\bmod (K, D):=\sup \{\bmod (A) ; A \subset D \text { e } A \text { é um anel em torno de } \mathrm{K}\}
$$

, dizer que $A$ é um anel em torno de $K$ significa que $K$ esta contido na componente limitada de $\mathbb{C}-A$.

Em particular quando $D-K$ conformemente isomorfo a um anel $A(R)$ teremos $\bmod (K, D)=$ $\bmod (D-K)$ e se $V$ for isomorfo a $\mathbb{C}$ ou $K$ for um ponto teremos $\bmod (K, D)=+\infty$.

Dizer que um anel $A$ ou uma renormalização $\left(P_{c}^{n}, U_{n}, V_{n}\right)$ tem módulo definido, ou que um subconjunto $U \subset \mathbb{C}$ tem área definida significa dizer que existe uma constante $0<\delta<+\infty$ talque $\bmod (A)>\delta>0, \bmod \left(U_{n}, V_{n}\right)>\delta>0$ ou área $(U)>\delta>0$, respectivamente.

Mencionaremos também a topologia de Carathéodory mais adiante, então, segue a definição: 
Definição 2.40. Chamaremos de domínio a um subconjunto $\Omega \subseteq \mathbb{C}$ que seja aberto e simplesmente conexo.

Consideremos a seguir o conjunto $\mathcal{D}$ de todos os domínios de $\mathbb{C},(U, u)$ com um ponto base $u \in U$. Isto posto, temos

Definição 2.41. A topologia de Carathéodory em $\mathcal{D}$ é definida por: $\left(U_{n}, u_{n}\right) \rightarrow(U, u)$ se, somente se

1. $u_{n} \rightarrow u$ quando $n \rightarrow+\infty$;

2. para todo compacto $K \subset U$ tivermos $K \subset U_{n}$ para todo $n$ suficientemente grande;

3. para qualquer aberto conexo $N$ contendo u tivermos $N \subset U_{n}$ para infinitos $n^{\prime} s$, então, $N \subset U$.

Seja $\mathcal{H}$ o conjunto das aplicações holomorfas $f:(U, u) \longrightarrow \mathbb{C} \operatorname{com}(U, u) \in \mathcal{D}$.

Definição 2.42. A topologia de Carathéodory em $\mathcal{H}$ é definida do seguinte maneira: Uma sequência de funções $\left\{f_{n}\right\}_{n \in \mathbb{N}}$ com $f_{n}:\left(U_{n}, u_{n}\right) \longrightarrow \mathbb{C} e\left(U_{n}, u_{n}\right) \in \mathcal{D}$ converge para $f:(U, u) \longrightarrow \mathbb{C}$ se, e somente se

1. $\left(U_{n}, u_{n}\right) \rightarrow(U, u)$ em $\mathcal{D}$;

2. $\left\{f_{n}\right\}_{n \in \mathbb{N}}$ converge para $f$ uniformemente nas partes compactas de $U$.

\subsubsection{Geodésicas fechadas em superfícies de Riemann hiperbólicas}

Definição 2.43 (Isotopia de curvas simples). Sejam $X$ uma superfície; $\alpha$ e $\beta$ curvas fechadas simples em $X$. Diremos que $\alpha$ e $\beta$ são isotópicas em $X$ se existir uma aplicação $H: \mathbb{S}^{1} \times[0,1] \longrightarrow X$ continua tal que $H(s, 0)=\alpha(s)$ e $H(s, 1)=\beta(s)$ para todo $s \in \mathbb{S}^{1}$ com a propriedade de que para cada $t \in[1,0] \alpha_{t}:=H(\cdot, t)$ seja simples.

A aplicação $H$, sem necessariamente possuir a propriedade de simplicidade, é chamada de homotopia livre. Assim, uma isotopia entre curvas simples nada mais é senão uma homotopia livre que para cada momento $t$ a curva deformada $\alpha_{t}$ obtida apartir de $\alpha$ ainda é uma curva simples. A condição de duas curvas fechadas simples serem ou não livremente homotópicas, isto é, de existir ou não uma homotopia livre entre elas induz uma relação de equivalência no conjuntos de todas tais curvas em $X$ para a qual denominamos as classes de equivalência de classes de homotopia livre.

Teorema 2.22 (Unicidade das geodésicas). Seja X uma superfície de Riemann hiperbólica com métrica hiperbólica $\rho_{X}$, e seja $\gamma$ uma curva primitiva simples em $X$. Então, existe três possibilidades:

1. existe uma única geodésica em $X$ homotópica a $\gamma$, ou

2. $\gamma$ é homotópica a um ponto(dita ser homotopicamente trivial), ou

3. $\gamma$ é homotópica a uma curva simples fechada $\gamma^{\prime}$ que é a fronteira de uma região $X^{\prime} \subset X$ isomorfa a $D^{*}:=D \backslash\{0\}$. Neste caso, $\gamma$ é chamada de curva periférica.

Teorema 2.23 (Geodésicas e posição minimal). 1. Se $\gamma \subset X$ é uma geodésica homotópica a uma curva simples e fechada $\gamma^{\prime}$, então, $\gamma$ é simples. 
2. Mais geralmente, o número de interseçôes entre duas geodésicas é igual ao menor número de interseções entre as curvas de suas classes de homotopia.

3. Toda geodésica simples fechada tem uma vizinhaça $U$ disjunta de todas as geodésicas simples e fechadas que nâa a intersecta. (Veja o Teorema da vizinhança colar-2.24 para um resultado mais preciso.)

Definição 2.44. Seja $\gamma$ uma geodésica simples e fechada de comprimento l em uma superfície de Riemann hiperbólica $X$. Se a $\delta$-vizinhança

$$
A_{\delta}(\gamma):=\{x \in X ; d(x, \gamma)<\delta\}
$$

é isometrica à $\delta$-vizinhança da única geodésia simples e fechada no cilindro de módulo $\frac{\pi}{l}$. Dizemos que $A_{\delta}(\gamma)$ é uma vizinhança colar de $\gamma$ ou que $\gamma$ admite uma vizinhaça colar.

Teorema 2.24 (Teorema da vizinhança colar). Seja $X$ uma superfície hiperbólica completa, $e$ seja $\Gamma:=\left\{\gamma_{1}, \gamma_{2}, \cdots\right\}$ uma coleção(finita ou não) de geodésicas simples, fechadas e disjuntas com comprimento $l_{i}$. Então as $\eta\left(l_{i}\right)$-vizinhanças $A_{\eta\left(l_{i}\right)}\left(\gamma_{i}\right)$ são vizinhanças colares das curvas $\gamma_{i}$, e são disjuntas. Em que $\eta$ é a função colar dada por $\eta(l):=\frac{1}{2} \log \frac{\cosh (l / 2)+1}{\cosh (l / 2)-1}$.

Consulte $[\mathrm{Hu}]$ para ver a construção da função colar. 


\section{Capítulo 3}

\section{Renormalização}

Trataremos neste capítulo de uma fortíssima ferramenta em sistemas dinâmicos de baixa dimensão que nos permite dentre muitos fins estudar a fina estrutura desses sistemas dinâmicos.

Comecemos, então, dizendo precisamente o que significa um polinômio quadrático ser renormalizável:

Definição 3.1. Considere um polinômio quadrático $P_{c} \operatorname{com} c \in \mathcal{M}$. Seja $n \in \mathbb{N}, P_{c}^{n}$ é renormalizável se existem discos topológicos $U$ e $V$ em $\mathbb{C}$ tais que o ponto crítico $0 \in U$ e $P_{c}^{n}: U \rightarrow V$ é uma aplicação do tipo quadrático cujo Julia cheio é conexo, ou equivalentemente, tal que $P_{c}^{n k}(0) \in U$ para todo $k \geq 0$. Se existe um tal $n \geq 2$ dizemos também que $P_{c}$ é renormalizável.

Isto é, $P_{c}$ é renormalizável se algum iterado seu tem uma restrição a uma vizinhança do seu ponto crítico $z=0$ que seja uma aplicação do tipo quadrático. E deste modo, pelo teorema de retificação de Douady-Hubbard(teorema 2.17) naturalmente temos um novo sistema dinâmico polinomial quadrático associado. Ou seja, recuperamos um novo sistema dinâmico na mesma classe do sistema dinâmico inicial dado por $P_{c}$. Isto é o que significa renormalizar. Isto posto, a escolha de um par $(U, V)$ como na definição 3.1 será denominado renormalização de $P_{c}^{n}$,ou como prefere John Milnor, é a $n$-ésima renormalização de $P_{c}$. Por vezes, nos referiremos a uma renormalização a denotando, também, por $\left(P_{c}^{n}, U, V\right)$. Note que o processo de renormalização induz um sistema dinâmico no espaço de parâmetro. Para tal abordagem consulte [AL], [L2], [L4].

Consideremos o conjunto

$$
\mathrm{R}(c):=\left\{n \in \mathbb{N} ; P_{c}^{n} \quad \text { é renormalizável }\right\}
$$

Aos inteiros positivos em $\mathrm{R}(c)$ chameremos de nivel da renormalização.

Se $|\mathrm{R}(c)|<\infty$ diremos que $P_{c}$ é finitamente renormalizável, caso contrário diremos que $P_{c}$ é infinitamente renormalizável.

Teorema 3.1. Quaisquer duas renormalizaçôes de $P_{c}^{n}$ possuem o mesmo Julia cheio.

Demonstração. Suponhamos $P_{c}^{n}: U \rightarrow V$ e $P_{c}^{n}: U^{\prime} \rightarrow V^{\prime}$ duas renormalizações de $P_{c}^{n}$ com respectivos Julia cheios $K$ e $K^{\prime}$. Como $K \subset K(c)$ é fechado e conexo, e $P_{c}^{n}: U^{\prime} \rightarrow V^{\prime}$ é uma aplicação tipo-polinômio, segue do teorema 6.13-[McM1] que $L=K \cap K^{\prime}$ é conexo. E temos ainda, $P_{c}^{n}(L)=L$, pois $P_{c}^{n}(K)=K$ e $P_{c}^{n}\left(K^{\prime}\right)=K^{\prime}$.

Consideremos a componente $\widehat{U}$ de $U \cap U^{\prime}$ que contém $L$, e ponhamos $\widehat{V}:=P_{c}^{n}(\widehat{U})$. Decorre, pois, do teorema 5.11-[McM1], que $P_{c}^{n}: \widehat{U} \rightarrow \widehat{V}$ é uma aplicação do tipo polinomial cujo Julia cheio é o 
conjunto $L$. E como o ponto crítico $0 \in L, P_{c}^{n}: \widehat{U} \rightarrow \widehat{V}$ tem grau igual à dois. Mas, já que o grau das três aplicações são iguais, segue, também do teorema 5.11-[McM1], que $K(c)=L=K=K^{\prime}$.

Suponhamos que para cada $n \in \mathrm{R}(c)$ temos escolhida uma renormalização

$$
P_{c}^{n}: U_{n} \rightarrow V_{n}
$$

(1) $P_{n}(n), J_{n}$ e $K_{n}$ denotarão os conjuntos pós-crítico, o conjunto de Julia e o Julia cheio da aplicação do tipo quadrático $P_{c}^{n}: U_{n} \rightarrow V_{n}$. Assumimos $J_{n}$ e $K_{n}$ conexos, assim temos $P_{n}(n) \subset K_{n}$;

(2) Aos conjuntos $K_{n}(i):=P_{c}^{i}\left(K_{n}\right)$ para $1 \leq i \leq n$ chamaremos de $i$-ésimo pequeno Julia cheio do nível $n$. Estes conjuntos são ciclicamente permutados por $P_{c}$, pois $P_{c}\left(K_{n}(i)\right)=P_{c}^{i+1}\left(K_{n}\right)=$ $K_{n}(i+1)$, em particular, $K_{n}(n)=K_{n}$;

(3) $P_{n}(i):=K_{n}(i) \cap \mathrm{P}(c)$ é o $i$-ésimo pequeno conjunto pós-crítico do nível $n$. Temos,

$$
\mathrm{P}(c)=\{\infty\} \cup \bigcup_{1}^{n} \mathrm{P}_{n}(i)
$$

(4) $J_{n}(i):=\partial K_{n}(i)$ é o $i$-ésimo pequeno Julia;

(5) $\mathcal{K}_{n}:=\bigcup_{1}^{n} K_{n}(i)$ é a união dos $n$ pequenos Julia cheios do nível $n$ da renormalização ;

(6) $\partial_{n}:=\bigcup_{1}^{n} J_{n}(i)$ é a união dos $n$ pequenos Julia do nível $n$ da renormalização ;

(7) $V_{n}(i):=P_{c}^{i}\left(U_{n}\right)$ para $1 \leq i \leq n$. Então, a aplicação tipo-quadrática $P_{c}^{n}$ fica fatorada como

$$
U_{n} \stackrel{P_{\subsetneq}}{\rightarrow} V_{n}(1) \stackrel{P_{\subsetneq}}{\rightarrow} \ldots \stackrel{P_{\subsetneq}}{\rightarrow} V_{n}(n)=V_{n}
$$

onde a aplicação $U_{n} \stackrel{f}{\rightarrow} V_{n}(1)$ é própria de grau 2 pois o ponto crítico $0 \in U_{n}$ e as demais aplicações são univalentes, visto que $P_{c}^{n}: U_{n} \rightarrow V_{n}$ tem grau igual à 2 ;

(8) $U_{n}(i)$ é a componente de $P_{c}^{i-n}\left(U_{n}\right)$ contida em $V_{n}(i)$. Veremos que $P_{c}^{n}: U(i) \rightarrow V(i)$ é uma aplicação do tipo quadrático;

(9) $P_{n}^{\prime}, J_{n}^{\prime}$ e $K_{n}^{\prime}$ são definidos por

$$
X_{n}^{\prime}:=-X_{n} \quad \text { para } \quad X \in\{P, J, K\}
$$

Como $P_{c}(-z)=P_{c}(z)$ cada um dos conjuntos $P_{n}^{\prime}, J_{n}^{\prime}$ e $K_{n}^{\prime}$ tem a mesma imagem por $P_{c}$ que seus conjuntos companheiros $P_{n}(n), J_{n}$ e $K_{n}$. E para $i \neq n$, relativamete a cada $i$, eles são disjuntos.

Note ainda que $K_{n}(i) \subset K(c)$, pois caso tenhamos $z_{0} \in K_{n}(i)-K(c)$, então, $\lim _{n \rightarrow \infty} P_{c}^{n}\left(z_{0}\right)=\infty$ o que nega o fato de que $P_{c}^{n k}\left(z_{0}\right) \in K_{n}(i)$ para todo $k \geq 0$.

Segue do Teorema 3.1 que o Julia cheio $K_{n}$, e por conseguinte, os conjuntos $K_{n}(i), J_{n}(i)$ e $\mathrm{P}_{n}(i)$ estão bem definidos para cada nível independendo da escolha do par $\left(U_{n}, V_{n}\right)$ 
Teorema 3.2. Suponha $P_{c}^{n}$ renormalizável. Então para cada $i=1, \ldots, n$

$$
P_{c}^{n}: U_{n}(i) \rightarrow V_{n}(i)
$$

é uma aplicação do tipo quadrático com Julia cheio igual à $K_{n}(i)$. Analogamente

$$
\left(-P_{c}^{n}\right): U_{n}^{\prime}(i) \rightarrow V_{n}^{\prime}(i)
$$

é uma aplicação do tipo qudrático com Julia cheio igual à $K_{n}^{\prime}(i)$. Ambas as aplicaçôes são conformemente conjugadas à $P_{c}^{n}: U_{n} \rightarrow V_{n}$.

Demonstração. Como $1 \leq n-i \leq n-1$ segue de (7) que a aplicação

$$
P_{c}^{n-i}: U_{n}(i) \rightarrow V_{n}(n)=V_{n}
$$

é univalente. $\left.P_{c}^{n-i}\right|_{U_{n}(i)}$ conjuga a aplicação $P_{c}^{n}: U_{n}(i) \rightarrow V_{n}(i)$ com a aplicação do tipo quadrático $P_{c}^{n}: U_{n} \rightarrow V_{n}$. Deste modo, $P_{c}^{n}: U_{n}(i) \rightarrow V_{n}(i)$ é uma aplicação do tipo quadrático. Como $\left.P_{c}^{n k}\right|_{U_{n}(i)}=\left.\left(P_{c}^{n-i}\right)^{-1} \circ f^{n k}\right|_{U_{n}} \circ P_{c}^{n-i}$ e $P_{c}^{n-i}\left(K_{n}(i)\right)=K_{n}$ segue

$$
\begin{aligned}
K\left(\left.P_{c}^{n}\right|_{U_{n}(i)}\right) & :=\left.\bigcap_{1}^{\infty} P_{c}^{-n k}\right|_{U_{n}(i)}\left(V_{n}(i)\right) \\
& =\left.\bigcap_{1}^{\infty} P_{c}^{i-n} \circ P_{c}^{-n k}\right|_{U_{n}} \circ P_{c}^{n-i}\left(V_{n}(i)\right) \\
& =\left.\bigcap_{1}^{\infty} P_{c}^{i-n} \circ P_{c}^{-n k}\right|_{U_{n}}\left(V_{n}\right) \\
& =P_{c}^{i-n}\left(\left.\bigcap_{1}^{\infty} P_{c}^{-n k}\right|_{U_{n}}\right) \\
& =f^{i-n}\left(K_{n}\right) \\
& =K_{n}(i)
\end{aligned}
$$

Analogamente, obtemos o mesmo para a aplicação $\left(-P_{c}^{n}\right): U_{n}^{\prime}(i) \rightarrow V_{n}^{\prime}(i)$, pois a aplicação univalente $\left(-P_{c}^{n-i}\right): V_{n}^{\prime}(i) \rightarrow V_{n}$ conjuga $\left(-P_{c}^{n}\right): U_{n}^{\prime}(i) \rightarrow V_{n}^{\prime}(i)$ com a aplicação do tipo quadrático $P_{c}^{n}: U_{n} \rightarrow V_{n}$ (veja o diagrama a baixo). E teremos, analogamente, $K\left(-\left.P_{c}^{n}\right|_{U_{n}^{\prime}(i)}\right)=K_{n}^{\prime}$.

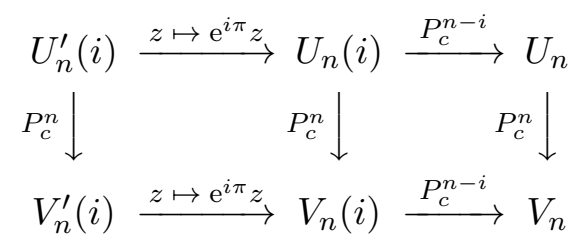

Teorema 3.3 (Conjuntos de Julia quase disjuntos). Seja $P_{c}^{n}$ renormalizável. Suponha que $K_{n}(i) e$ $K_{n}(j)$ sejam dois pequenos Julia cheios com interseção não vazia. Então,

$$
K_{n}(i) \cap K_{n}(j)=\{x\}
$$

e $x$ é um ponto repulsor de $P_{c}^{n}$. 
Demonstração. Seja $E:=K_{n}(i) \cap K_{n}(j)$. Como $P_{c}^{n}\left(K_{n}\right)=K_{n}$, então,

$$
\begin{aligned}
P_{c}^{n}(E) & \subset P_{c}^{n}\left(P_{c}^{i}\left(K_{n}\right)\right) \cap P_{c}^{n}\left(P_{c}^{j}\left(K_{n}\right)\right) \\
& =P_{c}^{i}\left(K_{n}\right) \cap P_{c}^{j}\left(K_{n}\right) \\
& =E
\end{aligned}
$$

$K_{n}(i)$ é conexo e está contido em $K(c)$, deste modo, segue do Teorema 6.13 que $E=K_{n}(i) \cap$ $K_{n}(j)$ é conexo, pois $P_{c}^{n}: U_{n}(j) \rightarrow V_{n}(j)$ é uma aplicação do tipo polinomial.

Sejam $W$ a componente de $U_{n}(i) \cap U_{n}(j)$ contendo $E=K_{n}(i) \cap K_{n}(j)$ e $W^{\prime}:=P_{c}^{n}(W)$.

Assim, pelo teorema 5.11-[McM1], $P_{c}^{n}: W \rightarrow W^{\prime}$ é uma aplicação do tipo polinomial. Como $K_{n}(i) \neq K_{n}(j)$, também decorre do teorema 5.11-[McM1] que $P_{c}^{n}$ tem grau igual a 1.

Seja $\psi:=\left(\left.P_{c}^{n}\right|_{W}\right)^{-1}: W^{\prime} \longrightarrow W^{\prime}$. Como $\psi\left(W^{\prime}\right)=W \Subset W^{\prime}$ segue do Teorema de Pick (teorema 2.11-[Mil1]) que $\psi$ decresce estritamente as distâncias hiperbólicas. Tomemos um compacto $C$ de modo que $E \subset C \subset W$, obtemos uma sequência $C_{0}:=C \supset C_{1}:=\psi\left(C_{0}\right) \cdots \supset C_{n}:=\psi\left(C_{n-1}\right) \cdots$ de compactos encaixados.Como $P_{c}^{n k}(E) \subset E$, temos $\psi^{k}(E) \supset E$, mas $\psi^{k}(C) \supset \psi^{k}(E)$ e, portanto, $E \subset \psi^{k}(C)=C_{k}$ para todo $k \in \mathbb{N}$, logo, $E \subset \bigcap_{0}^{\infty} C_{n}$ e $\bigcap_{0}^{\infty} C_{n} \neq \varnothing$.(O teorema de Cantor para interseção de compactos encaixados também nos dá $\left.\bigcap_{0}^{\infty} C_{n} \neq \varnothing\right)$.

Afirmação 3.1. diâm $\left(\bigcap_{0}^{\infty} C_{n}\right) \rightarrow 0$ na métrica hiperbólica.

Demonstração. Argumentaremos por contradição. Suponhamos que existam $z_{1} \neq z_{2}$ em $K:=$ $\bigcap_{0}^{\infty} C_{n}$ e tais que diâm $(K)=\mathrm{d}\left(z_{1}, z_{2}\right)$, isto é possivel devido à compacidade de $C$. Como $P_{c}(K)=$ $K$, escolhemos $w_{1}$ e $w_{2}$ em $K$ tais que $P_{c}\left(w_{1}\right)=z_{1}$ e $P_{c}\left(w_{2}\right)=z_{2}$.

Assim,

$$
\operatorname{diâm}(K)=\mathrm{d}\left(z_{1}, z_{2}\right)=\mathrm{d}\left(P_{c}\left(w_{1}\right), P_{c}\left(w_{2}\right)\right)<\mathrm{d}\left(w_{1}, w_{2}\right),
$$

uma contradição.

Portanto, $K$ consiste em apenas um único ponto $z_{0} \in W$ que é fixo para $\psi$ e, por conseguinte, $E=z_{0}$

Por fim, mostraremos que $z_{0}$ é repulsor para $P_{c}^{n}$. Para tanto, consideremos a aplicação de Riemann $\varphi: \mathbb{D} \longrightarrow W$ tal que $\varphi(0)=z_{0}$. Assim, obtemos uma aplicação holomorfa $G:(\mathbb{D}, 0) \longrightarrow$ $(\mathbb{D}, 0)$ dada por $G(z)=(\varphi)^{-1} \circ \psi \circ \varphi$.

Como, $G(\mathbb{D}) \Subset \mathbb{D}$, segue do Lema de Schwarz que $\left|G^{\prime}(0)\right|<1$, mas $G^{\prime}(0)=\left(\varphi^{-1}\right)^{\prime}\left(\psi\left(z_{0}\right)\right)$. $\psi^{\prime}\left(z_{0}\right) \cdot \varphi^{\prime}(0)=\frac{1}{\varphi^{\prime}(0)} \cdot \psi^{\prime}\left(z_{0}\right) \cdot \varphi^{\prime}(0)=\psi^{\prime}\left(z_{0}\right)$.

Portanto, como $\psi:=\left(\left.P_{c}^{n}\right|_{W}\right)^{-1}$, concluímos que $z_{0}$ é um ponto fixo repulsor para $P_{c}^{n}$.

Note que este teorema nos diz que o interior dos pequenos Julia cheios, que por suposição são conexos, são disjuntos.

Teorema 3.4. Suponhamos $P_{c}^{n}$ renormalizável. Então, qualquer ponto periódico atrator ou indiferente e qualquer componente periódica do interior de $K(c)$ está contido em $K_{n}(i)$ para um único $1 \leq i \leq n$. Seus períodos são divisiveis por $n$. 
Para uma prova do teorema acima consulte [McM1]. O seguinte corolário será importante.

Corolário 3.1. Todo ponto periódico de $P_{c}$ cujo período é menor do que n é repulsor.

Teorema 3.5 (Menor renormalização comum). Se $P_{c}^{n}$ e $P_{c}^{m}$ são renormalizáveis, então, assim o é $P_{c}^{l}$, onde l é o menor múltiplo comum de n e m. E mais, $K_{l}=K_{n} \cap K_{m}$.

Demonstração. Comecemos definindo o segunte conjunto

$$
U_{n}^{*}:=\left\{z \in U_{n} ; P_{c}^{n j}(z) \in U_{n} \quad \text { para } \quad j=1, \ldots,(l / n)-1\right\}
$$

Por ser $P_{c}^{n}$ renormalizável, temos $\mathrm{P}\left(\left.f^{n}\right|_{U_{n}}\right) \subset K_{n} \subset U_{n}$, assim $\left(P_{c}^{n}\right)^{-1}$ é localmente holomorfa em $V_{n}-U_{n}$, e portanto, $V_{n}-U_{n}$ é recoberto por $P_{c}^{-1}\left(V_{n}-U_{n}\right)$, logo, pela classificação das surperfícies de Riemann, $f^{-1}\left(V_{n}-U_{n}\right)$ também é um anel contido em $U_{n}$ cuja fronteira da componente ilimitada de seu complementar coincide com $\partial U_{n}$. Como anteriormente, podemos fazer o pullback do anel $A_{1}:=\left(P_{c}^{n}\right)^{-1}\left(V_{n}-U_{n}\right)$ por $\left(P_{c}^{n}\right)^{-1}$; e repetindo isto por mais $\frac{l}{n}-2$ vezes obtemos um anel $A_{\frac{n}{l}-1}$. Note que nenhum dos anéis $A_{k}$ 's tem interseção não vazia com $K_{n}$ visto que $P_{c}^{n}\left(K_{n}\right)=K_{n}$.

Assim, $A_{\frac{n}{l}-1} \cup B_{\frac{n}{l}-1}$, onde $B_{k}$ é a componente compacta de $\mathbb{C}-A_{k}$, é um disco tal que $P_{c}^{l}\left(A_{\frac{n}{l}-1} \cup B_{\frac{n}{l}-1}\right)=V_{n}$.

Vejamos agora que $A_{\frac{n}{l}-1} \cup B_{\frac{n}{l}-1}=U_{n}^{*}$.

Pela construção segue $A_{\frac{n}{l}-1} \cup B_{\frac{n}{l}-1} \subset U_{n}^{*}$. E $A_{\frac{n}{l}-1} \cup B_{\frac{n}{l}-1} \supset U_{n}^{*}$, pois caso exista $z \in U_{n}^{*}$ tal que $z \notin A_{\frac{n}{l}-1} \cup B_{\frac{n}{l}-1}$, como $U_{n}=B_{\frac{n}{l}-1} \cup \bigcup_{j=1}^{\frac{l}{n}-1} A_{j}$ existirá $j \in\left\{1, \ldots, \frac{l}{n}-1\right\}$ tal que $z \in A_{j}$, e assim teremos $P_{c}^{n\left(\frac{l}{n}-1\right)} \notin U_{n}$, o que contradiz que $z \in U_{n}^{*}$.

$P_{c}^{l}$ fica fatorada como

$$
\left(A_{\frac{n}{l}-1} \cup B_{\frac{n}{l}-1}\right) \stackrel{f^{n}}{\longrightarrow}\left(A_{\frac{n}{l}-2} \cup B_{\frac{n}{l}-2}\right) \stackrel{f^{n}}{\longrightarrow} \cdots\left(A_{1} \cup B_{1}=U_{n}\right) \stackrel{P_{c}^{n}}{\longrightarrow} V_{n}
$$

e $0 \in A_{j} \cup B_{j}$ para cada $j=1, \ldots, \frac{l}{n}-1$, concluímos que $P_{c}^{l}: U_{n}^{*} \longrightarrow V_{n}$ é uma aplicação do tipo polinomial de grau $d=2^{\frac{l}{n}}$.

Analogamente, $P_{c}^{l}: U_{m}^{*} \longrightarrow V_{m}$ é uma aplicação do tipo polinomial de grau $d=2^{\frac{l}{m}}$.

Desta maneira, pelo teorema 6.13i[McM1], $L=K_{n} \cap K_{m}$ é conexo, pois $K_{n} \subset K(c)$ é conexo e fechado. Pondo $U_{l}$ como a componente de $U_{n}^{*} \cap U_{m}^{*}$ que contém $L$ e $V_{l}:=P_{c}^{l}\left(U_{l}\right)$, seguirá do Teorema 5.11-[McM1] que $P_{c}^{l}: U_{l} \longrightarrow V_{l}$ é uma aplicação do tipo polinomial cujo Julia cheio é $L$.

Observe que se o ponto crítico 0 pertence à $P_{c}^{j}(L)$, então deve-se ter $j$ divisível por $n$ e por $m$, e portanto será um múltiplo de $l$, pois caso contrário chegaremos a contradição de que $0 \in$ $P_{c}^{j}\left(K_{n}\right)=P_{c}^{n d+r}\left(K_{n}\right)=K_{n}(r) \neq K_{n}$, com $r \in\{1, \ldots, n-1\}$. Por outro lado, se $n$ não divide $j$ e $m$ não divide $j$, então

$$
P_{c}^{j}\left(K_{n} \cap K_{m}\right) \subset K_{n}\left(r_{1}\right) \cap K_{m}\left(r_{2}\right)
$$

onde $r_{1} \equiv j \quad(\bmod \quad n)$ e $r_{2} \equiv j \quad(\bmod \quad m)$.

Mas, $K_{n} \cap K_{n}\left(r_{j}\right)=\left\{x_{j}\right\}$, com $x_{j}$ repulsor, ou $K_{n} \cap K_{n}\left(r_{j}\right)=\varnothing$, para $j=1,2$.

Então, $0 \notin P_{c}^{j}\left(K_{n} \cap K_{m}\right)$, pois $0 \in K_{n} \cap K_{m}$ é ponto crítico. Neste caso temos ainda,

$$
P_{c}^{j}\left(K_{n} \cap K_{m}\right)=K_{n} \cap K_{m}
$$


Decerto, pois $P_{c}^{j}\left(K_{n} \cap K_{m}\right) \subset P_{c}^{j}\left(K_{n}\right) \cap P_{c}^{j}\left(K_{m}\right)=K_{n} \cap K_{m}$, e por outro lado, se $z \in K_{n} \cap K_{m}$, então $P_{c}^{-1}(z) \subset K_{n}$ e $P_{c}^{-1}(z) \subset K_{m}$, pois $P_{c}^{-j}\left(K_{n}\right)=K_{n}$ e $P_{c}^{-j}\left(K_{m}\right)=K_{m}$. Logo, existe $w \in$ $K_{n} \cap K_{m} \cap P_{c}^{-1}$ tal que $P_{c}(w)=z$, e portanto, $P_{c}^{j}\left(K_{n} \cap K_{m}\right) \supset K_{n} \cap K_{m}$. Por notemos que assim $P_{c}^{l}$ possue apenas um ponto crítico em $L$, a saber o 0 , pois se tivermos $w \in L-\{0\}$ tal que $\left(P_{c}^{l}\right)^{\prime}(w)=\prod_{j=0}^{l-1} P_{c}^{\prime}\left(P_{c}^{j}(w)\right)=0$, então teremos $P_{c}^{\prime}\left(P_{c}^{j}(w)\right)=0$ para algum $j \in\{1, \ldots, l-1\}$. E neste caso teremos $P_{c}^{j}(w)=0$ implicando assim que $0 \notin K_{n} \cap K_{m}$, mas $0 \in K_{n} \cap K_{m}$. E como $L$ é connexo e $0 \notin K_{n} \cap K_{m}$, segue que $P_{c}^{l}: U_{l} \longrightarrow V_{l}$ tem grau igual a 2 , e portanto, $l \in \mathrm{R}(c)$ e $K_{l}=L=K_{n} \cap K_{m}$.

Corolário 3.2. Se $P_{c}^{n}$ e $P_{c}^{m}$ são renormalizáveis e $n \mid m$, então, $K_{n} \supset K_{m}$.

Demonstração. Com razão, pois se $n$ divide $m$, então, $\operatorname{mmc}(n, m)=m$. Logo, do teorema 3.5, decorre que $K_{m}=K_{n} \cap K_{m}$ e, portanto, $K_{n} \supset K_{m}$.

\subsection{Os pequenos conjuntos de Julia se intersectam em pontos pe- riódicos.}

A combinatória da renormalização é mais simples quando os pequenos conjuntos de Julias são disjuntos. Vimos que os pequenos conjuntos de Julia de um dado nível de renormalização se tocam apenas em pontos repulsores. Veremos que os períodos destes pontos de interseção dos pequenos conjuntos de Julia tendem para o infinito quando o nível da renormalização tende para o infinito.

O seguinte resultado é um bom substituto para a disjunção dos pequenos conjuntos de Julia.

Teorema 3.6 (períodos grandes). Dado um período p, existe apenas um número finito de niveis em $R(c)$ tais que o pequeno Julia cheio $K_{n}$ contém um ponto periódico de período $p$.

O ponto principal na demonstração deste resultado consiste em mostrar que para níveis $n \in \mathrm{R}(c)$ arbitrariamente grandes, $K_{n}$ não contém ponto fixo de $P_{c}$.

Para começarmos uma análise dos pontos fixos consideremos o polinômio quadrático $P_{c}$ com conjunto de Julia conexo e cujos pontos fixos sejam repulsores.

Na sequência obteremos alguns resultados preliminares que nos permitiram obter o teorema 3.6.

Teorema 3.7. Os raios externos que aterrissam em $\alpha$ são permutados transitivamente por $P_{c}$. $E$ os raios externos que aterrissam em $-\alpha$ separam $\beta$ do ponto crítico de $P_{c}$, isto é, $\beta$ e 0 pertencem a componentes diferentes do plano $\mathbb{C}$ menos os raios externos que aterrissam em $-\alpha$.

Demonstração. Pelo teorema 2.10 apenas um número finito de raios externos aterrissam em $\alpha$, ponto fixo repulsor de $P_{c}$. Se o raio $R_{t}$ aterrissa em $\alpha$, também aterrissa em $\alpha$ o raio externo $P_{c}\left(R_{t}\right)=R_{2 t}$. E como $P_{c}$ é injetora numa vizinhança de $\alpha$, segue que $P_{c}$ permuta os raios que aterrissam em $\alpha$. Pelo teorema 2.10 os raios que aterrissam em $\alpha$ possuem o mesmo período, e disto decorre que $P_{c}$ permuta tais raios de maneira transitiva.

Os raios que aterrissam em $\alpha$ são invariantes para a frente e dividem o plano complexo em $q$ componentes abertas $C_{1}, C_{2}, \cdots, C_{q}$, onde $q$ é o número de raios externos que aterrissam em $\alpha$, assumamos que a componente $P_{q}$ contenha o ponto crítico $z=0$ e o ponto $-\alpha$.

As pré-imagens dos raios que aterrissam em $\alpha$, aterrissam em $\alpha$ e $-\alpha$ dividindo o plano em $2 q-1$ componentes abertas $Q_{1}, Q_{2}, \cdots, Q_{2 q-1}$. Visto que apenas a peça $C_{q}$ é subdividida pelos 
raios externos que aterrissam em $-\alpha$, assumiremos a seguinte indexação para as componentes $Q_{i}$ 's:

$$
\begin{array}{r}
Q_{i}=C_{i}, \quad \text { para } 1 \leq i<q \\
\text { e } \quad Q_{q} \quad \text { contém o ponto crítico } z=0 .
\end{array}
$$

Como $P_{c}\left(C_{i}\right)$ é disjunto de $C_{i}$ para $i<q$, segue que $\beta \in C_{q}$ visto que $\beta$ é ponto fixo de $P_{c}$. $\beta$ também não pode estar em $Q_{q}$, pois como $P_{c}(-\alpha)=P_{c}(\alpha)=\alpha$ segue da continuidade de $P_{c}$ e conexidade de $Q_{q}$ que $P_{c}\left(Q_{q}\right)=C_{i}$ para algum $i<q$. Portanto, $\beta$ deve estar contido em alguma das componentes $Q_{i} \operatorname{com} q<i \leq 2 q-1$.

Teorema 3.8. Se $P_{c}^{n}$ é renormalizável e $n>1$, então o pequeno Julia cheio não contém o ponto fixo repulsor $\beta$ de $P_{c}$.

Demonstração. Argumentaremos por contradição.

Portanto, suponhamos que $\beta \in K_{n}$. Deste modo, $K_{n}(1)=P_{c}\left(K_{n}\right)$ também contém $\beta$, já que este é mantido fixado por $P_{c}$. Daí, como a interseção entre os $K_{n}$ 's é no máximo um ponto, $\alpha \notin K_{n}$. Assim, $K_{n}$ é disjunto dos raios externos que aterrissam em $-\alpha$, pois se $-\alpha \in K_{n}$, então, $\alpha=$ $P_{c}(-\alpha) \in K_{n}(1)$, e assim teremos $\alpha \in K_{n}$, pois $\alpha$ é ponto fixo de $P_{c}$ e $P_{c}^{n-1}\left(K_{n}(1)\right)=K_{n}$.

Mas pelo teorema anterior os raios que aterrissam em $-\alpha$ separam o ponto crítico 0 do ponto fixo $\beta, \operatorname{logo}, K_{n}$ não é conexo, e temos uma contradição.

Teorema 3.9. Suponha $P_{c}^{n}$ renormalizável para algum $n>1$ e $\alpha \in K_{n}$. Seja $\rho$ o número de componentes de $K_{n}-\{\alpha\}$. Então,

$$
n \rho \leq q
$$

em que q é o número de raios externos que aterrissam em $\alpha$.

Combinando os teoremas 3.8 e 3.9, obtemos:

Corolário 3.3. Para um nível $n \in \mathrm{R}(c)$ tal que $n>q$, o pequeno Julia cheio não contém os pontos fixos de $P_{c}$.

Decerto, pois $n>q>2$ e assim pelo teorema 3.9 concluímos que $\beta \notin K_{n}$. E $\alpha \notin K_{n}$, pois caso contrário teríamos ,pelo teorema 7.11,

$$
n \rho \leq q
$$

contradizendo, pois, que $n>q$.

Demonstração do teorema 3.9. Consideremos as $q$ componentes abertas do plano, $C_{i}$, determinadas pelos $q$ raios externos que aterrissam em $\alpha$. Assumamos que $C_{q}$ contenha o ponto crítico 0 e que $C_{1}$ contenha o valor crítico $c$. Como $C_{q}$ contém o ponto $-\alpha$, substituímos $C_{q}$ pela componente $C_{q}^{\prime}$ determinada pelos raios que outrora determinavam $C_{q}$ mais os raios que aterrissam em $-\alpha$ que são mapeados por $\mathrm{f}$ sobre os raios que determinam $C_{1}$. Assim, pelo teorema $3.7,\left\{C_{1}, C_{2} \cdots, C_{q}\right\}$ é mapeado transitivamente sobre si por $P_{c}$. Note que $P_{c}$ aplica $C_{q}$ sobre $C_{1}$ como um recobrimento crítico de grau 2.

Com isso, uma vez que $\alpha$ separa $K_{n}$ em $\rho$ componentes, vemos que o mesmo ocorre com $K_{n}(i)$ para cada $i=1, \cdots, n-1$. Portanto, $\mathcal{K}_{n}$ é separado por $\alpha$ em $n \rho$ componentes. Não obstante, como 
$K(c)$ é simplesmente conexo e por teorema 6.13-[McM1](princípio de conexidade) $\partial K_{n}(i) \subset \partial K(c)$ para cada $i \in 1, \cdots, n-1$, cada uma das $n \rho$ componentes de $\mathcal{K}_{n}-\alpha$ é também uma das componentes de $K-\alpha$, logo, pelo teorema 6.6-[McM1], $n \rho \leq q$.

Prova do teorema 3.6. Dado $p \in \mathbb{N}$. Como $P_{c}$ possue apenas um número finito de pontos periódicos com período $p$, que a saber são os zeros do polinômio $P_{c}^{p}(z)-z$, é suficiente mostrar que $w$, um ponto $p$-periódico de $P_{c}$, pode estar em apenas um número finito de pequenos Julia cheios $K_{n}$.

Isto é, se $w$ é um ponto periódico de $P_{c}$ de período $p$, então $w \in K_{n}$ somente para um número finito de níveis $n \in \mathrm{R}(c)$.

Para tanto, suponhamos que exista $a>p$ pertencente ao $\mathrm{R}(c)$ tal que $w \in K_{a}$. Assim, $w=$ $P_{c}^{p}(w) \in K_{a}(p)$, mas pelo Teorema $3.3,\{w\}=K_{a} \cap K_{a}(p)$ e $w$ é ponto fixo repulsor de $P_{c}^{a}$.

Seja $g$ o polinômio ao qual $P_{c}^{a}: U_{a} \longrightarrow V_{a}$ é hibridamente equivalente(pelo teorema de retificação 2.16).

Primeiramente suponhamos que $w$ corresponda ao ponto fixo $\beta$ de $g$.

Veremos que $w \notin K_{b}$ caso $b>a$.

O teorema da menor renormalização comum nos garante que $P_{c}^{d}$ é renormalizável e $K_{c}=K_{a} \cap K_{b}$ onde $d=m . m . c .(a, b)$.

Desta maneira, a renormalização de $P_{c}^{d}$ nos dá uma renormalização de $g^{\frac{d}{a}}$ via a conjugação

híbrida entre $P_{c}^{a}$ e $g$. Sendo $\frac{d}{a}>1$ segue do teorema 3.8 que $\beta$ não pertence ao Julia cheio de $g^{\frac{d}{a}}$. Assim, $w \notin K_{c}$ e, portanto, $w \notin K_{b}$, já que $w \in K_{a}$ e $K_{d}=K_{a} \cap K_{b}$.

Agora vejamos o caso em que $w$ corresponde ao $\alpha$, ponto fixo de $g$.

Seja $q$ o número de raios externos que aterrissam em $\alpha$. Segue do corolário 3.3 que $\alpha$ pertence a nenhum Julia cheio de renormalizações de $g$ cujo nível é superior a $q$. O que implica que $w \notin K_{b}$ para $b>a \dot{q}$, pois caso contrário, como $P_{c}^{d} \operatorname{com} d=$ m.m.c. $(a, b)$ é renormalizável, obteríamos como acima, uma renormalização de $g^{\frac{c}{a}}$, em que $\frac{d}{a}>q$ tal que $\alpha \in K\left(g^{\frac{d}{a}}\right)$. Uma contradição.

Portanto, temos o teorema demonstrado.

\subsection{Renormalização Simples}

A renormalização simples terá um papel fundamental no que se segue.

Definição 3.2. Considere $P_{c}$ e $n \in R(c)$. Para $1 \leq i \leq n, P_{c}^{n}: U_{n}(i) \longrightarrow V_{n}(i)$ é uma aplicação do tipo polinomial hibridamente equivalente a um polinômio quadrático $g_{i}$ com conjunto de Julia conexo. Os pontos fixos de $g_{i}$ serão denotados por $\alpha$ e $\beta$, onde $\beta$ corresponde ao ponto fixo no qual aterrissa o raio externo de ângulo 0 . Denotaremos por $\alpha_{n}(i)$ e $\beta_{n}(i)$ os pontos fixos correspondentes de $P_{c}^{n}$ em $K_{n}(i)$.

Pelo Teorema 3.3 se dois pequenos Julia cheios se tocam, então, tal interseção é apenas um ponto periódico repulsor de $P_{c}$. Com isso, se $K_{n}(i) \cap K_{n}(j)=\{p\}$ para $i \neq j$, então, $p=\alpha_{n}(i)$ ou $p=\beta_{n}(i)$.

Teorema 3.10 (mesmo tipo de renormalização). Todas as interseçôes entre os pequenos conjuntos de Julia de um mesmo nível ocorrem em um mesmo tipo de ponto fixo da renormalização. Isto é, nunca ocorre a situação na qual $K_{n}(i) \cap K_{n}(j)=\left\{\alpha_{n}(i)\right\} K_{n}\left(i^{\prime}\right) \cap K_{n}\left(j^{\prime}\right)=\left\{\beta_{n}\left(i^{\prime}\right)\right\}$. 
Demonstração. Temos $P_{c}\left(\alpha_{n}(i)\right)=\alpha_{n}(i+1)$ e $P_{c}\left(\beta_{n}(i)\right)=\beta_{n}(i+1)$, onde $n+1$ é equivalente ao 1 , já que, para $1 \leq i<n, P_{c}$ nos dá uma conjugação conforme entre $P_{c}^{n}: U_{n}(i) \longrightarrow V_{n}(i)$ e $P_{c}^{n}: U_{n}(i+1) \longrightarrow V_{n}(i+1)$. E para $i=n$ segue a igualdade devido $\alpha_{n}(i)$ e $\beta_{n}(i)$ serem pontos fixos de $P_{c}^{n}$, pois assim temos

$$
P_{c}\left(\alpha_{n}(n)\right)=P_{c}\left(P_{c}^{n-1}\left(\alpha_{n}(1)\right)\right)=P_{c}^{n}\left(\alpha_{n}(1)\right)=\alpha_{n}(1)
$$

Buscaremos obter uma contradição ao assumir que dois pequenos Julias cheios se intersectam em um ponto $\alpha_{n}(j)$ e outros dois que se intersectam em um ponto $\beta_{n}(k)$, todos de um mesmo nível.

Mas como $P_{c}$ permuta ciclicamente os conjuntos $K_{n}(i)^{\prime} s$ e os pontos $\alpha_{n}(i)$ e $\beta_{n}(i)$,então cada ponto $\alpha_{n}(i)$ e $\beta_{n}(i)$ pertence a pelo dois pequenos Julia cheios.

Concideremos então o grafo obtido a partir das interseções entre os pequenos Julia cheios nos pontos $\alpha_{n}(i)^{\prime}$ s e $\beta_{n}(i)^{\prime}$ s. Isto é, formemos o grafo $\Lambda$ cujos vértices são os pontos $\alpha_{n}(i)^{\prime} s$ e $\beta_{n}(i)^{\prime} s$ que são ligados por uma aresta apenas se pertence a um mesmo pequeno Julia.

Como $\Lambda$ contém mais arestas do que vértices, já que cada vértice pertence a pelo menos duas arestas, segue que $\Lambda$ contém pelo menos um cíclo $\left\langle K_{n}\left(i_{1}\right), K_{n}\left(i_{2}\right), \cdots K_{n}\left(i_{k}\right)\right\rangle$ com $k>1$ pois $\alpha_{n}(i) \neq \beta_{n}(i)$. Então, $L=K_{n}\left(i_{1}\right) \cup K_{n}\left(i_{2}\right) \cup \cdots \cup K_{n}\left(i_{k}\right)$ é conexo pois os conjutos adjacentes na união são conexos e se intersectam.

Mas $L \cap K_{n}\left(i_{1}\right)=\left\{\alpha_{n}(1), \beta_{n}(1)\right\}$ o que contradiz o teorema 6.13-[McM1] (pricipio de conexidade) que nos garante que $L \cap K_{n}\left(i_{1}\right)$ é conexo.

\subsection{Tipos de Renormalizações}

Um nível de renormalização $n \in \mathrm{R}(c)$ de $P_{c}$ pode ser classificada da seguinte maneira:

1. tipo $\alpha$, se algum par de pequenos conjuntos de Julia $\left(P_{c}^{n}, U_{n}, V_{n}\right)$ tem como interseção o ponto fixo $\alpha$ da renormalização;

2. tipo $\beta$, se algum par de pequenos conjuntos de Julia $\left(P_{c}^{n}, U_{n}, V_{n}\right)$ tem como interseção o ponto fixo $\beta$ da renormalização;

3. tipo disjunto, se todos os pequenos conjuntos de Julia $\left(P_{c}^{n}, U_{n}, V_{n}\right)$ são disjuntos.

Repare que o teorema 3.10 nos garante que tal classificação esta bem posta. Mais ainda, como o Julia cheio é o mesmo para um nível fixado independendo da renormalização $\left(P_{c}^{n}, U_{n}, V_{n}\right)$.

Definição 3.3. Diremos que uma renormalização é simples se for do tipo $\beta$ ou disjunto. E a chamaremos de cruzada caso contrário, isto é, se for do tipo $\alpha$.

Consideremos a seguinte notação

$$
\mathrm{SR}(c):=\left\{n \in \mathrm{R}(c) ;\left(P_{c}^{n}, U_{n}, V_{n}\right) \text { é simples }\right\}
$$

Teorema 3.11. Se $m \in \mathrm{R}(c)$ e $n \in \mathrm{SR}(c)$, então $m$ divide $n$ ou $n$ divide $m$.

Demonstração. Seja $d$ o máximo divisor comum entre $m$ e $n$. Se $d=\min \{m, n\}$, então, $m \mid n$ ou $n \mid m$.

Caso $d$ seja menor do que $m$ e $n$, teremos $K_{m}(d) \neq K_{m}$ e $K_{n}(d) \neq K_{n}$. 
Como $K_{m} \cap K_{n} \neq \varnothing$, já que $0 \in K_{m}$ e $0 \in K_{n}, P_{c}^{i}\left(K_{m}\right)$ e $P_{c}^{i}\left(K_{n}\right)$ também se intersectam para qualquer $i>0$. Em particular, $K_{m}(d) \cap K_{d} \neq \varnothing$. As sequências de conjuntos $\left\{P_{c}^{i}\left(K_{m}\right)\right\}_{i \in \mathbb{N}}$ e $\left\{P_{c}^{i}\left(K_{n}\right)\right\}_{i \in \mathbb{N}}$ são periódicas com períodos, $m$ e $n$, respectivamente, já que permuta ciclicamente o conjunto $\left\{K_{n}, K_{n}(1), \cdots, K_{n}(n-1)\right\}$ para cada nível $n \in \mathrm{R}(c)$. Da teoria elementar dos números, sabemos que existe $(k, l) \in \mathbb{Z} \times \mathbb{Z}$ tal que $k \cdot m+l \cdot n=d$, ou seja, existe um múltiplo de $n$ que é congruente ao $d$ módulo $m$, e vice-versa, existe um múltiplo de $m$ que é congruente ao $d$ módulo $n$. Isto implica que $K_{n}$ intersecta $K_{m}(d)$ e $K_{m}$ intersecta $K_{n}(d)$.

$L:=K_{n} \cup K_{n}(d) \cup K_{m}(d)$ é um fechado conexo contido em $K(c)$. O teorema 6.13[McM1](princípio de conexidade) nos garante que $K_{m} \cap L$ é conexo. Visto que $K_{m}(d) \cap K_{m}$ é no máximo um ponto, concluímos que $\left(K_{n} \cup K_{n}(d)\right) \cap K_{m}$, pois caso contrário, como $\left(K_{n} \cap K_{n}(d)\right) \cap K_{m}$ é fechado, assim o são os subconjuntos $A, B \subset\left(K_{n} \cap K_{n}\right) \cap K_{m}$ disjuntos tais $A \cup B=\left(K_{n} \cap K_{n}(d)\right) \cap K_{m}$. Portanto, $A$ e $B$ estão a uma distância positiva, o que implica que $K_{m} \cap L$ não pode ser conexo. Como tanto $K_{n}$ quanto $K_{n}(d)$ tem interseção não vazia com $K_{m}$, deve-se ter $K_{m} \cap K_{n} \cap K_{n}(d) \neq \varnothing$, pois caso contrário contradiremos novamente a conexidade de $K_{m} \cap L$, já que teríamos $K_{n}$ disjunto de $K_{m} \cap K_{n} \neq \varnothing$.

Mas, como $n \in \mathrm{SR}(c), K_{n}$ e $K_{n}(d)$ se intersectam no ponto fixo $\beta$ de $P_{c}^{n}$, e assim, $\beta \in K_{m} \cap K_{n}$.

O teorema 3.5 nos assegura que $P_{c}^{k}$ é renormalizável em que $k$ é o menor múltiplo comum de $m$ e $n$, com $K_{k}=K_{m} \cap K_{n}$. Mas deste modo, se $P_{x}$ é o polinômio quadrático ao qual $\left(P_{c}^{n}, U_{n}, V_{n}\right)$ é hibridamente equivalente, via o teorema de retificação 2.17 obteremos uma renormalização para $P_{x}$. Pela conjugação quaseconforme ente $\left(P_{c}^{n}, U_{n}, V_{n}\right)$ e $P_{x}$ obtemos uma renormalização para $P_{x}^{k / n}$ cujo conjunto de Julia contém o ponto fixo $\beta$ de $P_{x}$, o que contradiz o teorema 3.8 .

Corolário 3.4. O conjunto $\mathrm{SR}(c)$ é totalmente ordenado pela relação de divisibilidade.

Com o corolário 3.2 temos

Corolário 3.5. Os conjuntos $K_{n}$ formam uma sequência decrescente quando $n$ cresce através dos niveis em $\mathrm{SR}(c)$. Consequentemente, para cada par de níveis a e $b$ em $\operatorname{SR}(c)$ com $a<b$, cada pequeno Julia do nivel b estará contido em algum pequeno Julia do nivel a. tem-se o mesmo para os pequenos conjuntos pós-críticos.

O seguinte teorema será útil para a construção de renormalizações simples.

Teorema 3.12. Suponhamos $P_{c}^{a}$ simplesmente renormalizável e seja $P_{x}$ o polinômio ao qual $\left(P_{c}^{a}, U_{a}, V_{a}\right)$ é hibridamente equivalente. Se $P_{x}^{b}$ é renormalizável, então, assim o é a aplicação $P_{c}^{a \cdot b}$.

Demonstração. Seja $\Psi: W_{c} \longrightarrow W_{x}$ a aplicação quaseconforme que nos atesta que $\left(P_{c}^{a}, U_{a}, V_{a}\right)$ e $P_{x}$ são hibridamente equivalente. Trocando-se $V_{b}$ por sua pré-imagens por algum iterado de $P_{c}^{b}$, podemos assumir que $U_{b} \subset V_{b} \subset W_{x}$. E desta forma, $\left(P_{c}^{a \cdot b}, U_{a \cdot b}, V_{a \cdot b}\right)$, com $U_{a \cdot b}:=\Psi^{-1}\left(U_{b}\right)$ e $V_{a \cdot b}:=$ $\Psi^{-1}\left(V_{b}\right)$ nos dará uma renormalização de $P_{c}^{a \cdot b}$, pois $\Psi$ é um homeomorfismo e $P_{c}^{a \cdot b}=\Psi \circ P_{x}^{b} \circ \Psi^{-1}$, assim, $P_{c}^{a \cdot b}: U_{a \cdot b} \longrightarrow V_{a \cdot b}$ é própria e de grau igual a 2 com $U_{a \cdot b}$ compactamente contido em $V_{a \cdot b}$.

Destarte, nos resta verificar se $a \cdot b \in \mathrm{SR}(c)$. Vejamos isto.

Se $b=1,\left(P_{c}^{a \cdot b}, \Psi^{-1}\left(U_{b}\right), \Psi^{-1}\left(V_{b}\right)\right)$ será uma renormalização de $P_{c}^{a}$, logo é simples.

Se $b>1$, como $K\left(P_{x}^{b}\right) \subset K\left(P_{x}\right)$ e $\Psi^{-1}\left(K\left(P_{x}\right)\right)=K_{a}:=K\left(\left(P_{c}^{a}, U_{a}, V_{a}\right)\right)$, temos $K_{a \cdot b} \subset K_{a}(\mathrm{ou}$ mesmo pelo corolário 3.2). Temos ainda, $K_{a \cdot b}(i) \subset K_{a}\left(i^{\prime}\right)$.

Suponhamos, portanto, que $K_{a \cdot b}(i) \cap K_{a \cdot b}(j)=\left\{z_{0}\right\}$. Deste modo, $K_{a \cdot b}(i) \subset K_{a}\left(i^{\prime}\right)$ e $K_{a \cdot b}(j) \subset$ $K_{a}\left(j^{\prime}\right)$ para algum par de índices $i^{\prime}$ e $j^{\prime}$ em $\{1,2, \cdots, a\}$. Já que $P_{c}^{a}$ é simplesmente renormalizável, 
se $i^{\prime} \neq j^{\prime}$, então $z_{0}$ será o ponto fixo $\beta$ de $K_{a}\left(i^{\prime}\right)$, isto é, é o ponto fixo $\beta$ da aplicação do tipo quadrático $\left(P_{c}^{a}, U_{a}\left(i^{\prime}\right), V_{a}\left(i^{\prime}\right)\right)$. Mas assim, $K_{a \cdot b}(i)$ contém o ponto fixo $\beta$ de $K_{a}\left(i^{\prime}\right)$, o que contradiz o teorema 3.8 já que pelo teorema $3.2\left(P_{c}^{a}, U_{a}\left(i^{\prime}\right), V_{a}\left(i^{\prime}\right)\right)$ é conformemente conjugada $\left(P_{c}^{a}, U_{a}, V_{a}\right)$.

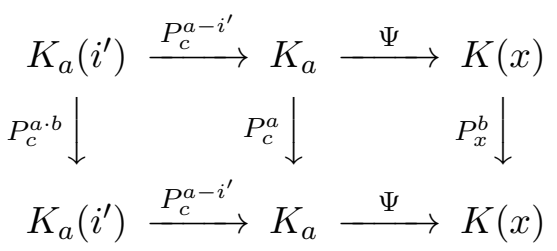

Portanto, $i^{\prime}=j^{\prime}$, e desde que $P_{c}: K_{a}(i) \longrightarrow K_{a}(i+1)$ é injetora para $i \in\{1, \cdots, a-1\}$ e $P_{c}^{a}: K_{a} \longrightarrow K_{a}$ é topologicamente conjugada à $P_{x}: K(x) \longrightarrow K(x)$. Note que $\Phi:=\Psi \circ P_{c}^{n-i^{\prime}}$ aplica $K_{a \cdot b}(i)$ em uma outra componente $K_{b}(l)$. Como $\Phi$ conjuga topologicamente $P_{c}: K_{a}(i) \longrightarrow K_{a}(i+1)$ à $P_{x}: K(x) \longrightarrow K(x)$ não podemos ter $K_{a \cdot b}(i)-K_{a \cdot b}(j)$ desconexo, pois se assim o tivermos contrariamos que $P_{x}^{b}$ é simplesmente renormalizável.

\subsubsection{Multiplicidade e Ramificação}

Se $P_{c}$ é renormalizável, a multiplicidade $m_{n}$ de um nível de renormalização $n \in \operatorname{SR}(c)$ é, por definição, o número dos pequenos conjuntos de Julia cheios do nível $n$ que intersectam $K_{n}$, incluindo o próprio $K_{n}$ na contagem. Note que a multiplicidade será igual a 1 se, e somente se, o nível de renormalização é do tipo disjunto, e que $m_{n}$ é igual ao número dos pequenos conjuntos de Julia em cada componente de $\mathcal{K}_{n}$.

A ramificação $\rho_{n}$ de um nível de renormalização $n \in \mathrm{SR}(c)$ é, por definição, o número de componentes do conjunto $K_{n}-\bigcup_{i \neq n} K_{n}(i)$. $\rho_{n}$ é igual ao número de componentes de $K_{n}(j)-$ $\bigcup_{i \neq j} K_{n}(i)$ para qualquer $j \in\{1,2, \cdots, n\} . \rho_{n}=1$ se, e somente se, a renormalização é do tipo simples. Decerto, pois $K_{n} \cap \bigcup_{i \neq n} K_{n}(i)$ é igual ao $\left\{\alpha_{n}(n)\right\}$, ao $\left\{\beta_{n}(n)\right\}$ ou $\varnothing$ conforme o tipo do nível de renormalização seja $\alpha, \beta$ ou disjunto. Mas para o tipo $\alpha$, sendo $\alpha_{n}(n)$ repulsor, teremos $\rho_{n}$ igual ao número de componentes de $K_{n}-\alpha_{n}(n)$ que é maor do 1.

\subsection{Polinômios infinitamente renormalizáveis}

O conjunto dos parâmetros $c \in \mathcal{M}$ para os quais $P_{c}$ é infinitamente renormalizável aparenta ser bastante magro. No entanto, estas aplicações são de central interesse, tanto pelas propriedades geométricas de seus conjuntos de Julia[Mil2][McM2] quanto ao fato de ainda não serem bem compreendidas. Por exemplo, os polinômios quadráticos infinitamente renormalizáveis são os únicos para os quais a conjectura sobre os campos de linhas invariantes permanece.

Fazendo-se uso do quebra-cabeça de Yoccoz mais os resultados das seções anteriores mostraremos a seguir que um polinômio infinitamente renormalizável $P_{c}$ admite uma infinidade de níveis para os quais a renormalização é simples. Encerraremos esta seção revisando alguns resultados devidos a Yoccoz, Lyubich e Shishikura sobre campos de linhas invariantes e polinômios quadráticos que são no máximo finitamente renormalizáveis.

Definição 3.4. Dizemos que $P_{c}$ é infinitamente renormalizável se $R(c)$ é infinito.

Teorema 3.13. Suponhamos $P_{c}$ infinitamente renormalizável. Então:

1. Toda órbita periódica de $P_{c}$ é repulsora; 
2. O interior de $K(c)$ é vazio(i.e., $K(c)=J(c))$;

3. $\bigcap_{R(c)} \partial_{n}$ não contém pontos periódicos;

4. O conjunto pós-crítico finito, $P(c) \cap \mathbb{C}$, não contém pontos periódicos;

5. Para qualquer $n \in R(c), P_{n}(i)$ e $J_{n}(j)$ são disjuntos se $i \neq j$.

Demonstração. Vimos no corolário 3.1 que se $P_{c}^{n}$ é renormalizável, então, qualquer ponto periódico de $P_{c}$ com período menor do $n$ é repulsor. Logo, se $P_{c}$ é infinitamente renormalizável teremos que qualquer órbita periódica de $P_{c}$ é repulsora.

Devido ao teorema de Sullivan de classificação das componentes de Fatou e o fato de que polinômios não possuem anel de Hermann, a existência de um aberto contido no Julia cheio de $P_{c}$ implica a existência de um cíclo atrator, parabólico ou irracionalmente indiferente, o que não ocorre devido ao item 1 provado acima. Com isso, temos $K(c)=J(c)$.

Do teorema 3.6 sabemos que fixado um período $p \in \mathbb{N}$, existe possivelmente um número finito de níveis $n \in \mathrm{R}(c)$ para os quais $K_{n}$ contém um ponto periódico de período igual a $p$, em particular, para os quais $J_{n}$ contém a órbita positiva de um ponto periódico de período $p$. Assim, se $x$ é um ponto periódico de $P_{c}$ a órbita de $x$ intersecta $J_{n}$ apenas para um número finito de níveis, logo é disjunto de $\mathcal{J}_{n}$ para todo $n$ suficientemerte grande e, por conseguinte, $n \in \mathrm{R}(\mathrm{c}) \mathcal{J}_{n}$. Portanto, $\bigcap_{\mathrm{R}(\mathrm{c})}$ não contém nenhum ponto periódico.

Visto que $\mathrm{P}(c) \cap \mathbb{C} \subset J_{n}$ para todo $n$, segue do que vimos logo acima que $\mathrm{P}(c) \cap \mathbb{C}$ também não contém ponto periódico de $P_{c}$.

Temos, $P_{n}(i) \subset K_{n}(i)$, mas pelo item $1, K_{n}(i)=J_{n}(i)$. Todavia, do teorema 3.3 , sabemos que se $K_{n}(i)$ e $K_{n}(j)$ se intersectam, então, tal interseção é um único ponto periódico de $P_{c}$. Desde que $\mathrm{P}(c) \cap \mathbb{C}$ não contém ponto periódico, deve-se ter $P_{n}(i) \cap P_{n}(j)=\varnothing$ para $i \neq j$.

Como no caso infinitamente renormalizável, $K_{n}=J_{n}$ para qualquer $n \in \mathrm{R}(c)$ e , portanto, $K_{n}(i)=J_{n}(i)$ e $\mathcal{K}_{n}=\mathcal{J}_{n}$ para qualquer $n \in \mathrm{R}(c)$, faremos uso apenas da notação com a letra $J$ para nos referirmos tanto ao Julia cheio quanto ao Julia.

Definição 3.5. Uma aplicação racional será ergódica se para qualquer subconjunto mensurável $A \subset \widehat{\mathbb{C}}$ para o qual $\left(P_{c}\right)^{-1}(A)=A$, então A tem medida(de Lebesgue) zero ou medida total.

Teorema 3.14 (dinâmica ergódica ou atratora). Se $R$ é uma aplicação racional com grau maior do que 1, então, $R$ satisfaz uma das seguintes opçôes:

1. o conjunto de Julia é toda a esfera de Riemann e a açâo de $R$ em $\widehat{\mathbb{C}}$ é ergódica; ou

2. $d\left(R^{n}(x), \mathrm{P}(R)\right) \rightarrow \infty$ para quase todo ponto $x$ no conjunto de Julia de $R$, quando $n \rightarrow \infty$, em que $\mathrm{P}(R)$ é o conjunto pós-crítico de $R$ e d e distância esférica.

Consulte [Mil1] para uma detalhada definição do conjunto de Julia e o conjunto pós-crítico de $R$.

Como consequência deste teorema temos o seguinte resultado:

Teorema 3.15 (Pequenos Julia atratores). Suponhamos $P_{c}$ infitamente renormalizável. Então, para qualquer $n \in R(c)$ e para quase todo ponto $x \in J(c)$, a órbita positiva de $x$ por $P_{c}$ entra no conjuto de Julia do nivel $n$, $\partial_{n}$. 
Demonstração. Como o Julia não é toda a esfera de Riemann decorre do teorema 3.14 que $d\left(P_{c}^{n}(x), \mathrm{P}(c)\right) \rightarrow$ 0 quando $n \rightarrow \infty$, para quase todo ponto $x$ em $J(c)$.

Em cada nível $n \in \mathrm{R}(c), \mathrm{P}(c)$ é particionado em $n$ peças compactas, $P_{n}(1), P_{n}(2), \cdots, P_{n}(n)$, que são permutadas ciclicamente por $P_{c}$. Assim, se $x$ fica arbitrariamente próximo de $\mathrm{P}(c)$ por iterados de $P_{c}$, ficará também arbitrariamente próximo de cada peça $P_{n}(i)$ de $\mathrm{P}(c) \cap \mathbb{C}$.

Deste modo, para todo $k$ suficientemente grande para os quais tenhamos $P_{c}^{k}(x)$ muito próximo de $P_{n}(n)$, teremos também $P^{k+n}(x)$ muito próximo de $P_{n}$. Logo, d $\left(P_{c}^{k+n j}(x), P_{n}(n)\right) \rightarrow 0$ quando $j \rightarrow \infty$, se fixamos um $k$ como acima.

Mas, visto que $P_{n}(n)$ é um subconjunto compacto de $U_{n}$, para $j$ grande o suficiente o ponto $y=P_{c}^{k+n j}(x)$ não escapa de $U_{n}$, o que implica que $y \in K_{n}=J_{n} \subset J_{n}$. E temos o resultado esperado.

\subsubsection{A tabela crítica}

Considere $P_{c} \operatorname{com} c \in \mathcal{M}$ cujos pontos fixos são repulsores e a órbita do ponto crítico seja disjunta do ponto fixo $\alpha$. Se um ponto $z \in K(c)$ tem sua órbita positiva disjunta do ponto fixo repulsor $\alpha$, então, existe uma única sequência de peças do quera-cabeça de Yoccoz de $P_{c}, Y_{0}(z) \supset Y_{1}(z) \cdots \supset$ $Y_{n}(z) \supset \cdots$.

A tabela associada a $z$ é uma ferramenta combinatória introduzida por Bodil Branner e $J$. $H$. Hubbard que nos permite representar graficamente a dinâmica da órbita de $z$ em relação a partição de $K(c)$ dada pelo quebra-cabeça de Yoccoz.

Definição 3.6. $J_{\alpha}(c):=\left\{z \in J(c) ; \exists n \in \mathbb{N}\right.$ tal que $\left.P_{c}^{n}(z)=\alpha\right\}$

Definição 3.7 (Nível semicrítico). Dado $z \in K(c)-J_{\alpha}(c)$, seja $\left\{Y_{d}(z)\right\}_{d \geq 0}$ a sequência de peças do quebra-cabeça que contém o ponto z. O nível semicrítico $S(z)$ de z é o maior inteiro $d \geq 0$ tal que $Y_{d}(z)=Y_{d}(0)$. Caso $Y_{d}(z)=Y_{d}(0)$ para todo nivel $d \geq 0$, definimos $S(z):=\infty$ e caso $P_{d}(z) \neq P_{d}(0)$ para todo $d \geq 0$, definimos $S(z)=1$. Assim, $S(z)$ será grande quando $z$ estiver próximo de $z=0$.

Para cada ponto $z \in K(c)$ podemos considerar a sequência de números inteiros $\left\{S\left(P_{c}^{n}(z)\right\}_{n \geq 0}\right.$. Esta sequência possui interressantes proprieddes.

Definição 3.8 (A tabela). A tabela $T(c, z)$ associada a um ponto $z \in K(c)-J_{\alpha}(c)$ é uma matriz $\mathbb{N} \times \mathbb{N}$ na qual a j-ésima coluna está associada ao ponto $z_{j}:=P_{c}^{j}(z)$ e a i-ésima linha está associada ao i-ésimo nível do quebra-cabeça de Yoccoz de modo que:

1. na j-ésima coluna é traçada uma linha vertical que vai do nível 0 , isto é, da linha 0 até o nivel $S\left(z_{j}\right)-1$;

2. e no nivel $S\left(z_{j}\right)$ é traçada duas linhas verticais na j-ésima coluna,

3. e do nivel $S\left(z_{j}\right)+1$ em diante é deixado em branco na j-ésima coluna.

Note que, em uma $j$-ésima coluna, um segmento de linha vertical duplo ou não em uma $i$-ésima linha da tabela indica que a peça do nível $j$ de quebra-cabeça que contém o ponto $z_{j}=P_{c}^{j}(z)$ é igual a peça crítica do nível $i$, isto é, $Y_{i}\left(z_{j}\right)=Y_{i}(0)$. Uma linha vertical longa indica que o ponto $z$ está próximo do ponto crítico. 


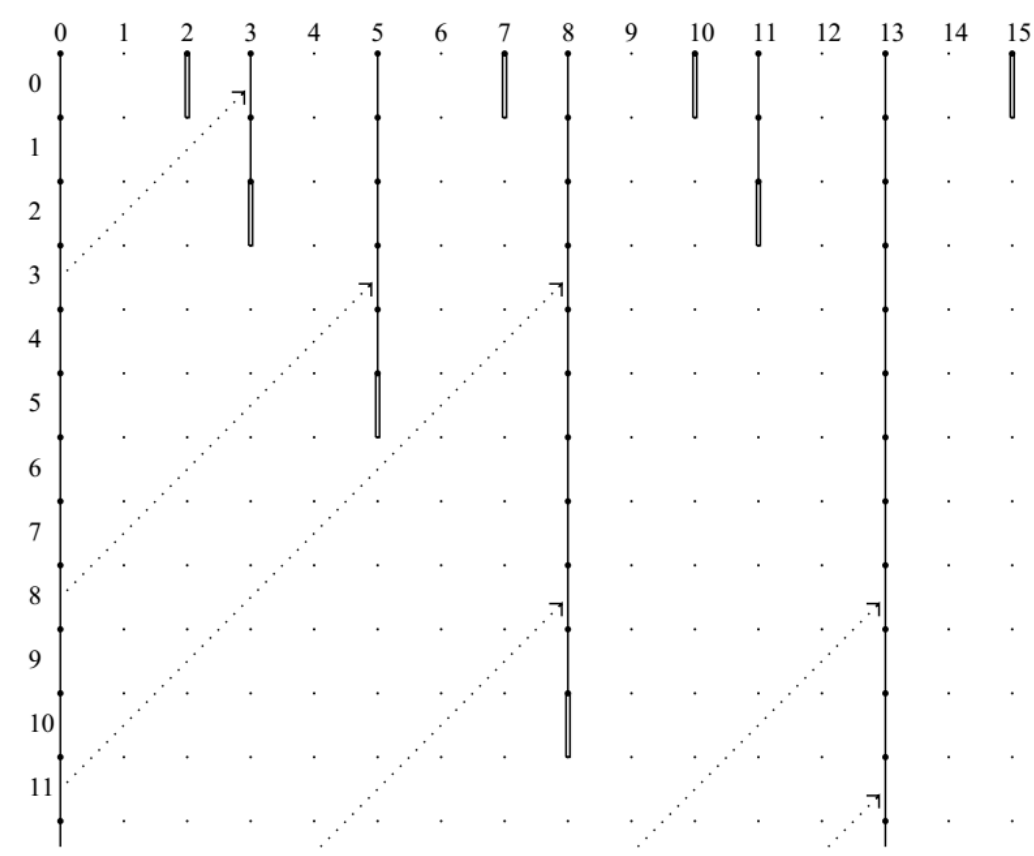

Figura 3.1: Tabela(tirada de [Mil2])

Uma diagonal de comprimento $m$ é uma diagonal traçada na tabela da esquerda para à direita e a partir de um nível $d \geq m$ até o nível $d+1-m$. Uma diagonal de comprimento $m$ corresponde à aplicação $P_{c}^{m-1}$ que envia a peça $Y_{d}\left(z_{j}\right)$ sobre a peça $Y_{d+1-m}\left(z_{j+m}\right)$.

Têm-se bastante interesse na tabela associada à órbita critica de $P_{c}$.

Definição 3.9 (Tabela crítica). A tabela crítica de um polinômio $P_{c}$, denotada por $T C(c)$, é a tabela associada à sua órbita crítica $\left\{P_{c}^{n}(0) ; n \geq 0\right\}$.

Definição 3.10 (período de $T C(c))$. $T C\left(\right.$ c) será periódica se existe $j \geq 0$ tal que $Y_{d}\left(P_{c}^{j}(0)\right)=Y_{d}(0)$ para todo nivel $d \geq 0$. O menor $j$ satisfazendo isto será, por definição, o período da tabela crítica de $P_{c}$. Se TC(c) não for periódica definimos o seu periodo como sendo infinito.

Temos uma definição de período para a renormalização também:

Definição 3.11. Se $S R(c) \neq \varnothing$, o período de renormalização de $P_{c}$ será o menor nível $n$ em $S R(c)$. Se $S R(c)=\varnothing$ definimos o período de renormalização de $P_{c}$ como sendo infinito.

Para mais informações sobre esta ferramenta consulte: [Mil2],[BrH], [J],[TY].

É um princípio geral em dinâmica holomorfa que o comportamento geral de um sistema dinâmico seja induzido pelo comportamento da órbita crítica. Por exemplo, o período da renormalização tem uma relação imediata com o tempo de retorno do ponto crítico à peça central:

Teorema 3.16. Considere $P_{c}$ com $c \in \mathcal{M}$ cujos pontos fixos são repulsores e a órbita do ponto crítico seja disjunta do ponto fixo $\alpha$. Então, o período da tabela crítica TC(c) é igual ao período de renormalização de $P_{c}$.

O seguinte teorema decorre de uma aplicação combinatória do quebra-cabeça de Yoccoz. Ele quem nos permitirá garantir que um polinômio quadrático infinitamente renormalizável admite uma infinidade de níveis de renormalizações simples. Consulte sua prova em [McM1]. 
Teorema 3.17. Consideremos $P_{c}$ com $c \in \mathcal{M}$, e sejam $E_{1}, E_{2}, \cdots, E_{n}$ subconjuntos conéxos $e$ fechados do Julia cheio $K(c)$ de $P_{c}$.

Se $0 \in E_{n}, P\left(E_{n}\right) \subset E_{1}$ e $P\left(E_{i}\right) \subset E_{i+1}$ para $i \in\{1,2, \cdots, n-1\}$, então, $P_{c}^{n}$ é simplesmente renormalizável.

Teorema 3.18. Se $P_{c}$ é infinitamente renormalizável, então, $P_{c}$ é simplesmente renormalizável para infinitos níveis $n \in \mathrm{R}(c)$, isto é, $|\mathrm{SR}(c)|=\infty$.

Demonstração. Para cada nível $n \in \mathrm{SR}(c)$ definimos $k_{n}$ como o número de componentes do Julia cheio do nível $n, \mathcal{K}_{n}$. Deste modo, $n=k_{n} \cdot m_{n}$. Lembre-se que $m_{n}$ é multiplicidade do nível $n$ (consulte a subseção 3.3.1).

Afirmação 3.2. $k_{n} \rightarrow \infty$ quando $n \rightarrow \infty$.

prova da afirmação 3.2. Dado $n \in \mathbb{N}$, se a renormalização de $P_{c}^{n}$ for do tipo disjunto, então, $k_{n}=n$. Caso contrário, consideremos o ponto $z_{0}$ onde os pequenos Julias cheios $K_{n}(i)$ de uma componente de $\mathcal{K}_{n}$ se intersectam. Do teorema $3.3, z_{0}$ é um ponto periódico repulsor de $P_{c}$. Assim, $k_{n}$ é igual ao período de $z_{0}$. Mas, o teorema 3.6 que o período de $z_{0}$ tende para o infinito. E, portanto, $k_{n} \rightarrow \infty$ quando $n \rightarrow \infty$.

Desta maneira, se provarmos que para cada $k_{n}, P_{c}^{k_{n}}$ é simplesmente renormalizável teremos o que queremos.

Decerto isto ocorre.

Tomemos os conjuntos $E_{i}$ do teorema 3.17 como sendo as componentes de $\mathcal{K}_{k_{n}}$ de modo que $0 \in$ $E_{k_{n}}$ e $P_{c}\left(E_{i}\right)=E_{i+1}$. Com as hipótese do teorema 3.17 e dele concluímos que $P_{c}^{k_{n}}$ é simplesmente renormalizável.

\subsection{Medida e conectividade local}

Teorema 3.19 (Yoccoz). Seja $P_{c}$ tal que

1. $J(c)$ é conexo;

2. $P_{c}$ não tem cíclo indiferente;

3. $P_{c}$ não é infinitamente renormalizável.

Então, $J(c)$ é localmente conexo.

Se, além do mais, $P_{c}$ não possui nenhum cíclo atrator, então, $c \in \partial \mathcal{M}$ e $\partial \mathcal{M}$ é localmente conexo em $c$.

Como consequência imediata do teorema acima temos:

Corolário 3.6. Se $J(c)$ suporta um campo de linhas invariante, então, $P_{c}$ é simplesmente renormalizável para uma infinidade de níveis $n \in \mathrm{R}(c)$. Em particular, $P_{c}$ é infinitamente renormálivel.

Teorema 3.20. Se os dois pontos fixos de $P_{c}$ são repulsores, então, ou $J(c)$ tem área zero ou $P_{c}^{n}$ é renormalizável para algum $n>1$. 


\section{Capítulo 4}

\section{Robustez}

Neste capítulo intruduziremos a noção de robustez de polinômios quadráticos devida à $M c M u l$ len, bem como os resultados necessários para a prova do Teorema de Rigidez. A robustez tem um caráter geométrico e para defini-la se faz necessário apresentarmos algumas definições e resultados geométricos.

Destarte, começaremos definindo um conjunto natural de geodésicas simples e disjuntas na superfície de Riemann hiperbólica $\widehat{\mathbb{C}}-\mathrm{P}(c)$. Os discos topológicos limitados por tais geodésicas cumprirão o papel dos intervalos básicos na construção do conjunto de Cantor. Diremos que um polinômio quadrático infinitamente renormalizável é robusto quando aquelas geodésicas tiverem comprimento limitado para uma quantidade infinita de níveis, com isso, mostraremos que o conjunto pós-crítico é um conjunto de Cantor com área zero e daremos à sua dinâmica um modelo topológico.

Estamos considerando apenas polinômios $P_{c}$ cuja $|\mathrm{P}(c)|=\infty$. Portanto, $\widehat{\mathbb{C}}-\mathrm{P}(c)$ admite uma estrutura de superfície de Riemann hiperbólica(vide-lema 2.5-[Mil1]).

Assim, pelo Teorema 3.18 sabemos que $|\mathrm{SR}(c)|=\infty$.

Definição 4.1 (Laços simples em torno do conjunto pós-crítico). Para cada nivel $n \in \mathrm{SR}^{*}(c), \gamma_{n}(i)$ denotará o representante geodésico na superfície hiperbólica $\widehat{\mathbb{C}}-\mathrm{P}(c)$ da classe de homotopia livre das curvas simples que separam o Julia $J_{n}(i)$ do resto do conjunto pós-crítico, isto é, de $\mathrm{P}(c)-J_{n}(i)$.

A existência de tais curvas separando o pequeno Julia $J_{n}(i)$ do resto do conjunto pós-crítico $\mathrm{P}(c)-J_{n}(i)$ decorre do Teorema 3.13 que nos garante que $P_{n}(j)$ e $J_{n}(i)$ são disjuntos se $j \neq i$. O fato de $n>1$ nos garante via o Teorema 2.22 a existência da geodésica $\gamma_{n}(i)$, já que um representante da classe de homotopia livre das curvas simples que separam $J_{n}(i)$ de $\mathrm{P}(c)-J_{n}(i)$ não poderá ser homotopicamente trivial nem periférico. E por fim, observamos que a unicidade das classes de homotopias livres decorem da conexidade de $J_{n}(i)$, pois se $J_{n}(i)$ não for conexo não é difícil exibir duas curvas que separam $J_{n}(i)$ de $P(c)-J_{n}(i)$ sem serem homotópicas. Denotaremos, $\gamma_{n}:=\gamma_{n}(n)$. Do Teorema 2.23 segue que as geodésicas $\gamma_{n}(i)$ são simples.

Teorema 4.1. As geodésicas $\Gamma:=\left\{\gamma_{n}(i) ; n \in \mathrm{RS}^{*}\left(P_{c}\right)\right.$ e $\left.1 \leq i \leq n\right\}$ são disjuntas.

Demonstração. Consideremos duas geodésicas $\gamma_{m}(i)$ e $\gamma_{n}(j)$. Podemos tomar representantes para as classes de homotopia livre de $\gamma_{m}(i)$ e $\gamma_{m}(i)$ em $\widehat{\mathbb{C}}-\mathrm{P}(c)$ arbitrariamente próximas de $J_{m}(i)$ e $J_{n}(j)$. Se os pequenos $J_{m}(i)$ e $J_{n}(j)$ são disjuntos, então, podemos escolher estes representantes de modo que sejam disjuntos, e desta maneira, pelo Teorema $2.23, \gamma_{m}(i)$ e $\gamma_{n}(j)$ são disjuntos. 
Suponhamos agora que $J_{m}(i)$ e $J_{n}(j)$ não sejam disjuntos. Se $m=n$, então, pelo Teorema $3.3, J_{m}(i) \cap J_{n}(j)=\left\{z_{0}\right\}$ com $z_{0}$ ponto periódico repulsor de $P_{c}$, que pelo Teorema (8.1-McM) não pertence ao conjunto pós-crítico. Já que a renormalização é simples, $z_{0}$ não disconecta $J_{m}(i)$ ou $J_{m}(j)$. Neste caso, como cada $\mathrm{P}_{m}(i)$ é compacto, existe $\varepsilon>0$ tal que $B\left(z_{0}, \varepsilon\right) \cap \mathrm{P}_{m}(i)=$ $\varnothing$ e $B\left(z_{0}, \varepsilon\right) \cap \mathrm{P}_{m}(j)=\varnothing$. Desta forma, conseguimos separar $J_{m}\left(i, z_{0}, \varepsilon\right)=J_{m}(i)-B\left(z_{0}, \varepsilon\right)$ de $\mathrm{P}(c)-J_{m}\left(i, z_{0}, \varepsilon\right)$ e $J_{m}\left(j, z_{0}, \varepsilon\right)=J_{m}(j)-B\left(z_{0}, \varepsilon\right)$ de $\mathrm{P}(c)-J_{m}\left(j, z_{0}, \varepsilon\right)$ por curvas simples disjuntas, $\operatorname{logo} \gamma_{m}(i)$ e $\gamma_{n}(j)$ são disjuntas.

Por fim, se $m<n$, então, pelo colorário temos $J_{n}(j) \subset J_{m}(k)$ para algum $k \in\{1,2, \cdots, m\}$.

Como as geodésicas $\gamma_{m}(i)$ são fronteiras de discos disjuntos, se $k \neq i$ temos $\gamma_{n}(j) \subset{\widetilde{Y_{m}}}_{m}(i)$ é disjunta de $\gamma_{m}(k)=\partial \widetilde{Y}_{m}(k)$, em que $\widetilde{Y}_{a}(b)$ é o interior de $\gamma_{a}(b)$ em $\mathbb{C}$.

Se $k=i$, teremos de garantir que $\gamma_{n}(j) \cap \gamma_{m}(k=i)=\varnothing$, já que $\gamma_{n}(j) \subset \widetilde{Y}_{m}(i)$. O que é equivalente a mostrarmos que existe um ponto de $\mathrm{P}(c)$ em $\mathrm{P}_{m}(i)-\mathrm{P}_{n}(j)$ já que o representante de cada classe de homotopia livre das curvas $\gamma_{a}(b)$ é único. Decerto isto ocorre, pois sendo $m<n$ temos $\mathrm{P}_{n}(j) \neq \mathrm{P}_{n}(j+m) \subset \mathrm{P}_{m}(i)$ e da definição de $\gamma_{n}(i)$ temos $\mathrm{P}_{n}(j+m) \subset \widetilde{Y}_{m}(i)-\overline{\widetilde{Y}}_{n}(j), \operatorname{assim}$, $\mathrm{P}_{n}(j+m) \subset \mathrm{P}_{m}(i)-\mathrm{P}_{n}(j)$ e temos o que queríamos.

Teorema 4.2 (Sistema de curvas invariantes). Dado $n \in R S^{*}\left(P_{c}\right)$. Para $i \neq 1,\left(P_{c}\right)^{-1}\left(\gamma_{n}(i)\right)$ tem uma componente $\alpha$ que é isotópica à $\gamma_{n}(i-1)$ em $\widehat{\mathbb{C}}-P(c)$ e cobre $\gamma_{n}(i)$ com grau 1 . A outra componente $\beta$ de $\left(P_{c}\right)^{-1}\left(\gamma_{n}(i)\right)$ é a fronteira de um disco disjunto do conjunto pós-crítico.

$E\left(P_{c}\right)^{-1}\left(\gamma_{n}(i)\right)=\alpha$ é uma curva isotópica à $\gamma_{n}$ e recobre $\gamma_{n}(1)$ com grau 2 .

Demonstração. Para $i \neq 1, J_{n}(i)$ não contém o valor crítico $P_{c}(0)$ e assim a pré-imagem de $\gamma_{n}(1)$ por $P_{c}$ é composta de duas componentes, uma destas é a componente $\alpha$ que é a fronteira de um disco que contém $J_{n}(i-1)$, enquanto a outra componente $\beta$ é a fronteira de um disco que contém $J_{n}^{\prime}(i-1)$, o pequeno conjunto de Julia companheiro de $J_{n}(i-1)$.

$\alpha$ separa $J_{n}(i-1)$ de $\mathrm{P}(c)-J_{n}(i-1)$, pois caso contrário teremos $p \in \mathrm{P}_{n}(j)$ na componente de $(\widehat{\mathbb{C}}-\mathrm{P}(c))-\alpha$ que contém $J_{n}(i-1)-\mathrm{P}_{n}(i-1)$ para algum $j \neq i-1$, já que $\mathrm{P}\left(P_{c}\right) \cap$ $\{\infty\}=\bigcup_{j=1}^{n} \mathrm{P}_{n}(j)$. O que contradiz que $\gamma_{n}(i)$ separa $J_{n}(i)$ de $\mathrm{P}\left(P_{c}\right)-J_{n}(i)$, pois $P_{c}(p) \in$ $\widetilde{Y}_{n}(i) \cap \mathrm{P}_{n}(j+1 \neq i)$. Portanto, $\alpha$ é isotópica à $\gamma_{n}(i-1)$. E como $J_{n}^{\prime}(i)$ está contido em $\widehat{\mathbb{C}}-\mathrm{P}(c)$, temos $B \cap \mathrm{P}(c)=\varnothing$, pois, cada $J_{n}^{\prime}(i)$ é disjunto de $\bigcup_{k=1}^{n} J_{n}(k)=\mathrm{P}(c)$. Portanto, $\beta$ é a fronteira de um disco em $\widehat{\mathbb{C}}-\mathrm{P}(c)$.

Quando $i=1, J_{n}(1)$ contém o valor crítico $P_{c}(0)$. E desta forma, $P_{c}^{-1}\left(\gamma_{n}(1)\right)=\alpha$ separa $J_{n}$ de $\mathrm{P}(c)-J_{n}$ pela razão exibida acima e, portanto, $\gamma_{n}(1)$ é isotópica à $\gamma_{n}$, e neste caso $\alpha$ é um recobrimento duplo de $\gamma_{n}(1)$ por $P_{c}$.

Teorema 4.3. Fixado um nível de renormalização simples $n>1$, os comprimentos das geodésicas $\gamma_{n}(1) ; 1 \leq i \leq n$, são comparáveis, e temos

$$
\frac{1}{2} l\left(\gamma_{n}\right) \leq l\left(\gamma_{n}(1)\right) \leq \cdots \leq \gamma_{n}(n-1) \leq l\left(\gamma_{n}\right)
$$

Demonstração. Consideremos o conjunto $Q:=P_{c}^{-1}(\mathrm{P}(c))$.

Pelo Teorema da Função Inversa $P_{c}: \widehat{\mathbb{C}}-Q \longrightarrow \widehat{\mathbb{C}}-\mathrm{P}(c)$ é um homeomorfismo local, sendo assim, uma aplicação de recobrimento, que por sua vez, via teorema de Pick(2.11-[McM1]), implica 
que $P_{c}$ é uma isometria local com respeito a metrica hiperbólicas em $\widehat{\mathbb{C}}-Q$ e $\widehat{\mathbb{C}}-\mathrm{P}(c)$. Lembre-se que $|\mathrm{P}(c)|>+\infty$.

Também por Pick a inclusão $i: \widehat{\mathbb{C}}-Q \hookrightarrow \widehat{\mathbb{C}}-\mathrm{P}(c)$ decresce estritamente a distância hiperbólica.

Lembre-se, $P_{c}^{-1}\left(\gamma_{n}(i)\right)$, para $1 \leq i \leq n$ tem uma componente $\alpha \approx_{i s o t} \gamma_{n}(i-1)$ (teorema anterior).

$$
\begin{aligned}
l\left(\gamma_{n}(i-1)\right) & \leq l(\alpha) \quad(\text { pois } \alpha \text { é o representante geodésico de }[\alpha] ;) \\
& \leq l_{\widehat{\mathbb{C}}-Q}(\alpha) \quad(\text { pois } i \text { é contração; }) \\
& =l\left(\gamma_{n}(i)\right) \quad\left(P_{c}(\alpha)=\gamma_{n}(i) \quad \text { e } P_{c} \text { isometria local } 1-1 .\right)
\end{aligned}
$$

Por fim, $P_{c}^{-1}\left(\gamma_{n}(1)\right)=\alpha$ é isotópico à $\gamma_{n}$ e recobre $\gamma_{n}(1)$ com grau dois, e disto resulta,

$$
l\left(\gamma_{n}\right) \leq l(\alpha) \leq l_{\widehat{\mathbb{C}}-Q}(\alpha)=2 l\left(\gamma_{n}(1)\right)
$$

\subsection{1 Área do conjunto pós-crítico}

Definição 4.2. Um polinômio quadrático infinitamente renormalizável $P_{c}$ será chamado robusto se

$$
\liminf _{\mathrm{SR}^{*}} l_{\mathbb{H}}\left(\gamma_{n}\right)<\infty
$$

no qual $l(\cdot)$ denota o comprimento hiperbólico em $\widehat{\mathbb{C}}-\mathrm{P}(c)$.

A robustez, como já mencionado, é uma propriedade do conjunto pós-crítico. Destarte, com relação à robustez, os pequenos conjuntos de Julia desempenham um papel apenas combinatório quanto a escolha das curvas simples $\gamma_{n}(i)$.

O seguinte teorema decorrerá da aplicação do teorema da vizinhaça colar 2.24.

Teorema 4.4 (conjunto pós-crítico com medida zero). Suponhamos $P_{c}$ robusto. Entâo,

1 o conjunto pós-crítico $\mathrm{P}(c)$ é um conjunto de Cantor com medida zero;

2 quando $n \rightarrow+\infty$ em $\mathrm{SR}^{*}(c)$;

$3 P_{c}: \mathrm{P}(c) \longrightarrow \mathrm{P}(c)$ é um homeomorfismo topologicamente conjugado à aplicação

$$
\sigma: x \mapsto x+1
$$

agindo no grupo profinito

$$
\Lambda:=\underset{n \in \operatorname{SR}(c)}{\operatorname{proj} \lim } \mathbb{Z} / n
$$

Demonstração. Enumeremos $\mathrm{SR}^{*}(c)=\{n(1), n(2), \cdots\}$ com $n(k)<n(k+1)$.

Para cada $k \in \mathbb{N}$ e $i=1, \cdots, n(k)$, consideremos as vizinhanças colares $A_{k}(i):=C\left(\gamma_{k}(i)\right)$ em torna das geodésicas $\gamma_{n}(i)$ na superfície hiperbólica $\widehat{\mathbb{C}}-\mathrm{P}(c)$.

Pelo teorema 2.24, as vizinhanças colares são disjuntas; e $A_{k}(i)$ separa o pequeno conjunto pós-crítico $\mathrm{P}_{n(k)}(i)$ de $\mathrm{P}(c)-\mathrm{P}_{n(k)}(i)$, pois o teorema nos garante $A_{k}(i) \hookrightarrow \widehat{\mathbb{C}}-P(c)$ e $\gamma_{n(k)}(i)$. 
Definimos $E_{k}$ para cada $k \in \mathbb{N}$ como sendo a união das vizinhanças colares do nível $n(k)$, isto é, $E_{k}=\bigcup_{i=1}^{n(k)} A_{k}(i)$.

Pelo corolário 3.5 o pequeno conjunto pós-crítico $\mathrm{P}_{n(k+1)}(i)$ está contido em algum $\mathrm{P}_{n(k)}(j)$. Como, pelo teorema da vizinhança colar, os anéis que compõe $E_{k+1}$ são disjuntos dos anéis em $E_{k}$, decorre que cada componente $A_{n(k+1)}(i)$ fica contida na componente de $(\widehat{\mathbb{C}}-\mathrm{P}(c))-A_{n(k)}(j)$ que não contém $\infty$, para algum $j \in\{1, \cdots, n(k)\}$. E desde que para cada $n(k) \in \mathrm{RS}^{*}, \mathrm{P}(c)=\bigcup_{i=1}^{n(k)} \mathrm{P}(i)$, então para cada $p \in \mathrm{P}(c)$ existe uma sequência $\left\{i_{n(k)}\right\}$ de números naturais tal que $p \in \mathrm{P}_{n(k)}\left(i_{n(k)}\right)$, para a qual $i_{n(k)} \in \mathrm{SR}^{*}(c)$. Desta forma, temos $p \in D_{i_{n(k)}}$ para cada $i_{n(k)}$, em que $D_{i_{n(k)}}$ é a componente $\widehat{\mathbb{C}}-A_{n(k)}(i)$ que não contém $\infty$.

Por ser $P_{c}$ ser robusto, $\liminf \operatorname{insR}_{n \in \mathrm{SR}^{*}(c)} l\left(\gamma_{n}\right)<\infty$. Do teorema 4.3 sabemos que o comprimentos hiperbólico das geodésicas $\gamma_{n(k)}(i)$ são limitados superiormente pelo comprimento hiperrbólico da geódesica $\gamma_{n(k)}$, para $n(k) \in \mathrm{SR}^{*}(c)$ fixado. Desta maneira, obtemos a partir do teorema 2.19-[McM1] concluímos que para um ponto arbitrário $p \in \mathrm{P}(c)$ a série $\sum_{k=1}^{\infty} \bmod \left(D_{i_{n(k)}}\right)$ diverge, pois existe subsequência $\left\{l\left(\gamma_{n\left(k_{j}\right)}\right)\right\}$ de $\left\{l\left(\gamma_{n(k)}\right)\right\}$ tal que $\gamma_{n\left(k_{j}\right)}<L$, para algum $L \in(0,+\infty)$, e disto decorre

$$
\begin{aligned}
\sum_{k=1}^{\infty} \bmod \left(D_{i_{n(k)}}\right) & \geq \sum_{j=1}^{\infty} \bmod \left(D_{i_{n\left(k_{j}\right)}}\right) \\
& \geq \sum_{j=1}^{\infty} \frac{M(L)}{2}=\infty
\end{aligned}
$$

Por conseguinte, o teorema 2.16-[McM1] implica que $D=\bigcap_{k \geq 1} D_{k}$ é totalmente disconexo e tem medida de Lebesgue nula. Temos também $D=\mathrm{P}(c)$, pois $\mathrm{P}(c) \subset D_{k}$ para cada $k \in \mathbb{N}$, isto é, $\mathrm{P}(c) \subset \bigcup_{k \geq 1} D_{k}$, e por outro lado, se $x \in \bigcap_{k \geq 1} D_{k}$, então, $x=\bigcap_{j \geq 1} D_{k_{j}}$, mas $p=\bigcap_{k \geq 1} \mathrm{P}_{n(k)}\left(i_{k_{j}}\right) \subset$ $\bigcap_{k \geq 1} D_{k}$ e, portanto, $x=p \in \mathrm{P}(c)$. Note ainda que por ser $D$ totalmente disconexo, o maior diâmetro das componentes de $D_{k}$ tende para zero. Isto porque, se não, existirá uma sequência decrescente $\left\{D_{k_{j}}\right\}_{j \in \mathbb{N}}$ tal que diâm $\left(D_{k_{j}}\right) \not \rightarrow 0$ o que contradirá que $\bigcap_{j \geq 1} D_{k_{j}}=\{p\} . \operatorname{Assim}, \sup _{i} \operatorname{diâm}\left(\mathrm{P}_{n(k)}(i)\right)$ tende para zero.

Para cada nível $n \in \mathrm{SR}^{*}(c)$ temos bem definida a aplicação $f_{n}: \mathrm{P}(c)=\bigcup_{i=1}^{n} \mathrm{P}_{n}(i) \longrightarrow \mathbb{Z} / n$ que associa $\mathrm{P}_{n}(i)$ ao $i \bmod n$.

Se $m>n$ são inteiros $\operatorname{com} m=q \cdot n$ para cada $i \in\{0,1,2, \cdots, n-1\}$, vale:

$$
[i]_{n}=\bigcup_{k=1}^{q}\left[j_{k}\right]_{n} \quad \text { tal que } \quad j_{k} \equiv i \quad \bmod n
$$

em que $[i]_{n}$ denota um elemento de $\mathbb{Z} / n$ e $j_{k} \in 0,1, \cdots, m-1$.

Esta aplicação induz um homomorfismo

$$
\begin{aligned}
& \varphi_{n m}: \mathbb{Z} / m \longrightarrow \mathbb{Z} / n \\
& {[j]_{m} \longmapsto[i]_{n} \quad \text { se } \quad j \equiv i \bmod n }
\end{aligned}
$$

Com isso, podemos ver $\left((\mathbb{Z} / n) ;\left(\varphi_{n m}\right)\right)$ como um sistema inverso com respeito ao $\operatorname{SR}^{*}(c)$ (veja [B1],[B2]) cujo limite inverso é exatamento o subconjunto $\Lambda:=\left\{x=\left(x_{1}, x_{2}, \cdots\right) \in \prod_{n \geq 2} \mathbb{Z} / n ; x_{m}=\right.$ $\left.\varphi_{n m}\left(x_{n}\right)\right\}$. 
As aplicações $f_{n}: \mathrm{P}(c)=\bigcup_{i=1}^{n} \mathrm{P}_{n}(i) \longrightarrow \mathbb{Z} / n$ induzem uma aplicação $\varphi: \mathrm{P}(c) \longrightarrow \Lambda$ dada por $\varphi\left(z_{0}\right)=\left(f_{n(1)}\left(z_{0}\right), f_{n(2)}\left(z_{0}\right), f_{n(3)}\left(z_{0}\right), \cdots\right) \in \Lambda$. Estas aplicações são compatíveis no seguinte sentido,

$$
\varphi_{n m}\left(f_{m}\right)=f_{n}
$$

Disto decorre a continuidade de $\varphi$ com respeito a topologia inicial(ou projetiva). Note também que $\varphi$ é sobrejetora, isto por construção, e é injetora devido o fato de que cada sequência encaixada dos pequenos conjuntos pós-críticos converge para um ponto. E, portanto, $\varphi$ é bijetora.

Temos na teoria dos grupos profinitos uma caracterização destes grupos que nos garantem que estes são espaços topológicos compactos Hausdorff e totalmente disconexos, isto é, são um conjunto de Cantor. Por conseguinte, sendo $\mathrm{P}(c)$ compacto, $\Lambda$ Hausdorff e $\varphi$ bijeção continua, $\varphi$ é na verdade um homeomorfismo. E disto concluímos que $\mathrm{P}(c)$ é,também, um conjunto de Cantor.

Dado $z_{0} \in \mathrm{P}(c)$, temos

$$
\begin{aligned}
\sigma\left(\varphi\left(z_{0}\right)\right) & =\varphi\left(z_{0}\right)+1 \\
& =\left(f_{n(1)}\left(z_{0}\right)+[1]_{n(2)}, f_{n(2)}\left(z_{0}\right)+[1]_{n(2)}, f_{n(3)}\left(z_{0}\right)+[1]_{n(3)}, \cdots\right) \\
& =\left(f_{n(1)}\left(P_{c}\left(z_{0}\right)\right), f_{n(2)}\left(P_{c}\left(z_{0}\right)\right), f_{n(3)}\left(P_{c}\left(z_{0}\right)\right), \cdots\right) \\
& =\varphi\left(P_{c}\left(z_{0}\right)\right)
\end{aligned}
$$

Ou seja, visto que $P_{c}\left(\mathrm{P}_{n}(i)\right)=\mathrm{P}_{n}(i), \varphi$ conjuga $\left.P_{c}\right|_{\mathrm{P}(c)}$ à $\sigma$, isto é, o seguinte diagrama comuta.

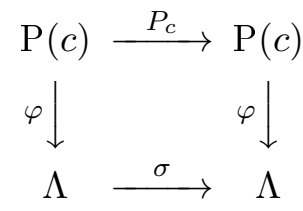

Parece pouco provável que todo polinômio quadrático infinitamente renormalizável sejá robusto. Douady e Hubbard apresentaram um exemplo no qual $P_{c}(z)=z^{2}+c$ é infinitamente renormalizável e, no entanto, $J(c)$ não é localmente conexo, sustentando isso (consulte:§3 - [Mil2]). É conjecturado que a robustez implica a conexidade local do Julia ,ou mesmo, medida de Lebesgue zero. Há também o problema sobre a conexidade local do Mandelbrot em um parâmetro robusto. Note que se a órbita crítica de um polinômio infinitamentte renormalizável $P_{c}$ for densa em $J(c)$, então, tal polinômio não poderá ser robusto. 


\section{Capítulo 5}

\section{Rigidez}

Neste capítulo provaremos o mais importante resultado deste trabalho, o teorema de rigidez cujo enunciado é

Teorema 5.1 (Rigidez robusta). Um polinômio quadrático robusto não suporta em seu conjunto de Julia um campo de linhas invariante.

Devido aos teoremas 2.19 e 2.20 o teorema acima é equivalente a rigidez quasiconforme dos polinômios quadráticos robustos, isto é, que qualquer polinômio quadrático $P_{a}$ que é quaseconformemente conjugado a um polinômio $P_{c}$ robusto é, então, conformemente equivalentes a $P_{c}$, ou seja, $a=c$. Por tal razão nos referimos a tal resultado como resultado de rigidez, já que passamos por cima da equivalência mais fraca até a mais forte, mas desde que a primeira se verifique.

A prova do teorema de rigidez enunciado acima será dividida em dois casos que, a saber, seram quando $L=0$ ou $L>0$.

A prova será por contradição e seguirá um padrão geral de argumentações e construções.

Suporemos que um polinômio qudrático rubosto suporte sobre o seu conjunto de Julia um campo de linhas invariante $\mu$. A hipótese de robustez nos garantirá uma certa compacidade. Com isto, para obter uma contradição tomaremos uma subsequência de níveis $n \in \mathrm{SR}(c)$ para a qual, depois de uma normalização, $P_{c}^{n}$ convergirá em uma vizinhança do pequeno conjunto pós-critico $P_{n}(n)$ a um sistema dinâmico limite $P_{\infty}$ que será uma aplicação própria de grau igua a 2.

Em seguida, construiremos a partir de $\mu$ um campo de linhas univalente invariante por $P_{\infty}$. Mas isto não pode ocorrer, pois $P_{\infty}$ tem um ponto crítico. Logo, um polinômio quadrático robusto não pode suportar em seu conjunto de Julia um campo de linhas invariante.

\subsection{Primeira Parte}

Começamos com a seguinte definição:

Definição 5.1. Admitamos $P_{c}^{n}$ renormalizável. Diremos que uma renormalização $P_{c}^{n}: U_{n} \longrightarrow V_{n}$ é regular se

$$
V_{n} \cap \mathrm{P}(c)=P_{n}(n)
$$

Teorema 5.2. Se $P_{c}^{n}: U_{n} \longrightarrow V_{n}$ é uma renormalização regular, então, $V_{n}^{\prime}(i)$ é disjunto do conjunto pós-crítico de $P_{c}$ para $i \neq n$. 
Demonstração. Temos, $P_{c}\left(P_{n}(i)\right) \subset P_{n}(n+1)$, e disto tiramos, $V_{n}(i) \cap P(c) \subset P_{n}(1)$, pois

$$
\begin{aligned}
V_{n}(i) \cap P(c) & =P_{c}^{i}\left(V_{n}\right) \cap P_{c}^{i} P(c) \subset P_{c}^{i}\left(V_{n} \cap P(c)\right) \\
& =P_{c}^{i}\left(P_{n}(n)\right)=P_{n}(i)
\end{aligned}
$$

Desde que $P_{c}\left(V_{n}(i)\right)=P_{c}\left(V_{n}^{\prime}(i)\right) \supset P_{n}(i)$ e como, devido aos teoremas 3.3 e 8.1-[McM1] (parte ainda $n$ digit.), em cada nível os pequenos conjuntos pós-críticos são disjuntos para $P_{c}$ infinitamente renormalizável, decorre que os pontos de $V_{n}^{\prime}(i) \cap P(c)$ estão em $P_{n}(i)$, pois caso contrário, digamos $x \in V_{n}^{\prime}(i) \cap P_{n}(j)$, então, $P_{c}(x) \in P_{n}(j+1)$, mas $P_{c}(x) \in P_{n}(i+1)$ e contradiremos o ítem 4 do teorema 3.13. Não obstante, $V_{n} \supset P_{i}(i)$ é disjunto de $V_{n}^{\prime}$, logo, $V_{n}^{\prime}$ não contém pontos de $\mathrm{P}(c)$, se $i \neq n$, pois $0 \in V_{n} \cap V_{n}^{\prime}$.

E como $\mathrm{P}(c)$ é positivamente invariante e $V_{n}^{\prime}(i) \cap \mathrm{P}(c)=\varnothing, P_{c}^{-k}\left(V_{n}\right) \cap \mathrm{P}(c)=\varnothing$ para $k>0$, podemos definir $P_{c}^{-k}$ sendo univalente sobre $V_{n}(i)$ para todo $k>0$. Por isso denominamos a renormalização de regular.

\subsubsection{Rigidez de aplicações do tipo polinomial}

Teorema 5.3. Suponhamos que a renormalização de $P_{c}^{n}$ é regular com $\bmod \left(U_{n}, V_{n}\right)>m>0$. Então, os pequenos conjuntos de Julia companheiros $J_{n}^{\prime}(i)$ para $i \neq n$ satisfazem

$$
\operatorname{diâm}_{\mathbb{H}}\left(J_{n}^{\prime}(i)\right) \leq C(m) .
$$

Demonstração. Sendo $\bmod \left(U_{n}, V_{n}\right)$ é limitado inferiormente segue do teorema 2.4-[McM1] que o diâmetro de $U_{n}$ é limitado superiormente na métrica hiperbólica de $V_{n}$. E, como $J_{n} \subset U_{n}$, o diâmetro de $J_{n}$ também é limitado naquela métrica por uma constante $C(m)$. Do teorema 3.2, $V_{n}-U_{n}$ é conformemente isomorfo ao anel $V_{n}^{\prime}(i)-U_{n}^{\prime}(i)$, assim $C(m)$ também limita o diâmetro hiperbólico de $U_{n}^{\prime}(i)$ e $J_{n}^{\prime}(i)$ em $V_{n}^{\prime}(i)$. Mas, pelo teorema $5.2, V_{n}^{\prime}$ é disjunto de $\mathrm{P}(c)$ e pelo teorema de Pick(2.11-[Mil1]) a inclusão $i: V_{n}^{\prime} \hookrightarrow \widehat{\mathbb{C}}-\mathrm{P}_{c}$ decresce a distância hiperbólica. Com isto, $C(m)$ também limita o diâmetro hiperbólico de $U_{n}^{\prime}(i)$ e $J_{n}^{\prime}(i)$ em $\widehat{\mathbb{C}}-\mathrm{P}(c)$.

Teorema 5.4. Se $P_{c}$ possue uma infinidade de níveis de renormalização simples regular satisfazendo

$$
\bmod \left(U_{n}, V_{n}\right)>m>0
$$

então, $P_{c}$ é robusto e

$$
\mathrm{P}(c) \cap \mathbb{C}=\bigcap_{\operatorname{SR}(c)} \mathcal{J}_{n}
$$

Demonstração. Suponhamos $P_{c}^{n}$ simplesmente renormalizável com $\bmod \left(U_{n}, V_{n}\right)>m>0$. Então, a curva central $\alpha$ do anel $V_{n}-\overline{U_{n}}$ tem comprimento hiperbólico neste anel(veja[McM1]pg.12)

$$
l_{\mathbb{H}}(\alpha)=\frac{\pi}{\bmod \left(U_{n}, V_{n}\right)} \leq \frac{\pi}{m} .
$$

Nos é garantido pelo corolário 7.15-[McM1] que cada componente de $\mathcal{J}_{n+1}$ fica contida em alguma componente de $\partial_{n}$ para cada $n \in \mathrm{SR}(c)$. Sendo $\alpha$ homotópica à $\gamma_{n}$ em $\widehat{\mathbb{C}}-\mathrm{P}(c), l_{\mathbb{H}}\left(\gamma_{n}\right)<l_{\mathbb{H}}(\alpha)<\frac{\pi}{m}$, 
$\operatorname{logo}, \liminf \operatorname{seSR}_{n(c)} l_{\mathbb{H}}\left(\gamma_{n}\right) \leq \frac{\pi}{m}<+\infty$. Assim, do teorema 4.4, $\mathrm{P}(c)$ é um conjunto de Cantor com medida de Lebesgue nula com $\sup _{i} \operatorname{diâm}_{\mathbb{E}} \rightarrow 0$ quando $n \rightarrow+\infty$ em $\mathrm{SR}^{*}(c)$.

Seja $n \in \mathrm{SR}^{*}(c)$ tal que $\bmod \left(U_{n}, V_{n}\right)>m>0$. Por mesmo argumento dado na demonstração anterior, $\bmod \left(U_{n}(i), V_{n}(i)\right)=\bmod \left(U_{n}, V_{n}\right)$, portanto, $\bmod \left(U_{n}(i), V_{n}(i)\right)>m>0$. Mas o teorema $(8.1-[\mathrm{McM} 1])$ nos garante que $P_{c}$ não possue ponto periódico atrator. Em razão disto, do corolário 5.10-[McM1], segue

$$
\operatorname{diâm}_{\mathbb{E}}\left(J_{n}(i)\right) \leq C(m) \operatorname{diâm}_{\mathbb{E}} P_{n}(i) .
$$

Logo, $\operatorname{diâm}\left(J_{n}(i)\right) \rightarrow 0$ quando $n \rightarrow+\infty$ em $\mathrm{SR}^{*}(c)$, e disto segue que cada componente de $\bigcap n \in \mathrm{SR}^{*}(c) \mathcal{J}_{n}$ é um único ponto, isto é, $\bigcap_{S R(c)} \mathcal{J}_{n}$ é totalmente disconexo. Já que cada componente de $\mathcal{J}_{n}$ intersecta $\mathrm{P}(c)$ para todo $n \in \mathrm{R}(c)$, então, $\mathrm{P}_{c} \cap \mathbb{C}=\bigcap_{S R(c)} \mathcal{J}_{n}$.

Corolário 5.1. Assumindo as hipóteses do teorema anterior temos para quase todo ponto $x \in J(c)$ as seguintes propriedades:

1. a órbita positiva de $x$ é disjunta do conjunto pós-crítico;

2. $\left\|\left(P_{c}^{n}\right)^{\prime}(x)\right\| \rightarrow+\infty$ na métrica hiperbólica em $\widehat{\mathbb{C}}-\mathrm{P}(c)$;

3. para qualquer $n \in \mathrm{SR}(c)$, existe um inteiro $k>0$ tal que $P_{c}^{k}(x) \in \mathcal{J}_{n}$;

4. para cada inteiro $k>0$ existe um $n \in \mathrm{SR}(c)$ para o qual $P_{c}^{k} \notin \mathcal{J}_{n}$.

Demonstração. Toda aplicação holomorfa não constante é discreta, isto é, a pré-imagem de um ponto é um conjunto discreto do domínio da função, em particular é enumerável. Assim, sendo $\mathrm{P}(c)$ enumerável e de medida zero, $\cup_{n=0}^{\infty} P^{-n}(\mathrm{P}(c))$. Mas note que $\cup_{n=0}^{\infty} P^{-n}(\mathrm{P}(c))$ é exatamente o conjunto dos pontos cuja órbita positiva em algum momento entra em $\mathrm{P}(c)$ e temos o item 1 .

O item 2 segue do item 1 mais o teorema 3.6-[McM1], ao passo que o item 3 decorre diretamente do teorema 3.15 .

Pelo teorema 5.4, $\mathrm{P}_{c} \cap \mathbb{C}=\bigcap_{S R(c)} \mathcal{\partial}_{n}$. Pelo corolário 3.5, $\mathcal{J}_{n+1} \subset \mathcal{J}_{n}$ para cada $n \in \mathrm{SR}(c)$ e pelo teorema 4.4 a medida de $\mathrm{P}(c)$ é igual a zero. Deste modo, em razão, da continuidade inferior da medida decorre que a medida de $\mathcal{J}_{n}$ tende a zero quando $n \rightarrow \infty$ em $\operatorname{SR}(c)$. Agora, fixando $k>0$, temos

$$
\begin{aligned}
\operatorname{área}\left(P_{c}^{-k}\left(\mathcal{J}_{n}\right)\right) & \leq 2^{k} \int_{\mathcal{J}_{n}}\left|\operatorname{Jac}\left(P_{c}^{k}\right)(z)\right|(\rho(z))^{2}|\mathrm{~d} z|^{2} \\
& \leq 2^{k} M_{n_{1}} \operatorname{área}\left(\mathcal{J}_{n}\right)
\end{aligned}
$$

em que $M_{n_{j}}:=\operatorname{máx}\left\{\left|\operatorname{Jac}\left(P_{c}^{k}\right)(z)\right| ; z \in \mathcal{J}_{n_{j}}\right\}$ e $n_{j} \in\left\{n_{1}, n_{2}, \cdots\right\}=\operatorname{SR}(c)$. E como $\mathcal{J}_{n_{j+1}} \subset \mathcal{J}_{n_{j}}$ para cada $n_{j} \in \mathrm{SR}(c), M_{n_{1}} \geq M_{n_{j}}$ para todo $j \geq 1$. Disto, segue que a área de $\bigcap_{n \in \operatorname{SR}(c)} \partial_{n}$ é zero, pois $P_{c}^{-k}\left(\mathcal{J}_{n_{j+1}}\right) \subset P_{c}^{-k}\left(\mathcal{J}_{n_{j}}\right)$.

Definição 5.2. Para um polinômio quadrático $P_{c}$ definimos

$$
\operatorname{USR}(c, m)=\left\{n \in \operatorname{SR}(c) ; P_{c}^{n}: U_{n} \longrightarrow V_{n} \text { é regular e } \bmod \left(U_{n}, V_{n}\right)>m>0\right\} .
$$

Teorema 5.5 (Rigidez de aplicações do tipo polinomial). Se existe uma constante $m>0$ tal que $|\operatorname{USR}(c, m)|=+\infty$. Então, o conjunto de Julia de $P_{c}$ não suporta um campo de linhas invariante por $P_{c}$. 
Demonstração. Suponhamos $\operatorname{USR}(c, m)$ infinito e que $P_{c}$ admite um campo de linhas invariante $\mu$ suportado em um conjunto $E \subset J(c)$ com medida positiva.

Seja $x \in E$ um ponto de quasecontinuidade de $\mu$ que também satisfaz as propriendades garantidas pelo corolário 5.1.

Para cada $n \in \mathrm{SR}(c)$ seja $k(n) \geq 0$ o menor inteiro não negativo tal que $P_{c}^{k(n)+1}(x) \in \mathcal{J}_{n}$.

Devido a nossa escolha de $x \in J(c), k(n) \rightarrow+\infty$ quando $n \rightarrow+\infty$, pois, caso contrário, o conjunto $I=\left\{k(n) \in \mathbb{N} ; P_{c}^{k(n)+1}(x) \in \mathcal{J}_{n} \operatorname{com} n \in \mathrm{SR}(c)\right\}$ é finito. E assim, tomando $p=$ $\prod_{k_{i} \in I}\left(k_{i}+1\right)$ teremos $P_{c}^{p}(x) \in \mathcal{J}_{n}$ para todo $n \in \mathrm{SR}(c)$ o que contradiz o item 4 do corolário 5.1.

Consideremos $n \in \operatorname{USR}(c, m)$ suficientemente grande de modo que $k(n)>0$. Com isso, $P_{c}^{k(n)+1}(x) \in$ $\partial_{n}$ e $P_{c}^{k(n)}(x) \notin \mathcal{J}_{n}$, já que, do contrário, contradizemos a minimalidade de $k(n)>0$ e, portanto, $P_{c}^{k(n)}(x) \in J_{n}^{\prime}(i(n))$ para algum $i(n)=1,2, \cdots, n-1$. Note ainda que $P_{c}^{k(n)+1}(x) \in J_{n}(1)$, pois, neste caso, teríamos $P_{c}^{k(n)}(x) \in J_{n}(n) \subset \mathcal{J}_{n}$.

Visto que a renormalização é regular, existe um ramo univalente de $P_{c}^{-k(n)}$ definido em $V_{n}^{\prime}(i(n))$ que aplica $P_{c}^{k(n)}(x)$ de volta ao $x$.

Sendo a aplicação $P_{c}^{k(n)}: V_{n}^{\prime} \longrightarrow V_{n}$ é univalente, definimos a aplicação também univalente, $h_{n}$, da seguinte maneira:

$$
h_{n}: V_{n}^{\prime} \stackrel{P_{c}^{i(n)-n}}{\longmapsto} V_{n}^{\prime}(i(n)) \stackrel{P_{c}^{-k(n)}}{\longmapsto} \mathbb{C}
$$

Pela invariância suposta do campo $\mu,\left.\mu\right|_{V_{n}}=h_{n}^{*}(\mu)$. Seja, $J_{n}^{*}=h_{n}\left(J_{n}\right)=P_{c}^{-k(n)}\left(J_{n}^{\prime}(i(n))\right)$, a pequena cópia do Julia contendo $x$.

$\mathrm{Na}$ demonstração do teorema 5.3 vimos que o diâmetro de $U_{n}^{\prime}(i(n))$ é limitado na métrica hiperbólica de $\widehat{\mathbb{C}}-\mathrm{P}(c)$ e sabemos que $\left\|P_{c}^{k(n)}(x)\right\| \rightarrow+\infty$. Disto decorre que $\operatorname{diâm}_{\mathbb{H}}\left(J_{n}^{*}\right) \rightarrow 0$, pois para quaisquer dois pontos $z, w \in J_{n}^{*}$ e $\gamma$ uma curva ligando $z$ a $w$ em $P_{c}^{-k(n)}\left(U_{n}^{\prime}(i(n))\right) \subset \widehat{\mathbb{C}}-\mathrm{P}(c)$

$$
\mathrm{d}_{\mathbb{H}}(z, w) \leq l_{\mathbb{H}}(\gamma)=\int_{\gamma^{\prime}}\left\|\left(P_{c}^{-k(n)}\right)^{\prime}(w)\right\| \rho(w)|d w|,
$$

em que $\gamma^{\prime}=P_{c}^{k(n)}(\gamma)$, mas, pelo teorema 3.8-[McM1],

$$
\frac{1}{C(m)} \frac{1}{\left\|\left(P_{c}^{k(n)}\right)^{\prime}(x)\right\|} \leq\left\|\left(P_{c}^{-k(n)}\right)^{\prime}(w)\right\| \leq \frac{C(m)}{\left\|\left(P_{c}^{k(n)}\right)^{\prime}(x)\right\|},
$$

e, portanto,

$$
\mathrm{d}_{\mathbb{H}}(z, w) \leq \frac{C(m)}{\left\|\left(P_{c}^{k(n)}\right)^{\prime}(x)\right\|} \operatorname{diâm}_{\mathbb{H}}\left(P_{c}^{-k(n)}\left(U_{n}^{\prime}(i(n))\right)\right) \leq \frac{C(m)}{\left\|\left(P_{c}^{k(n)}\right)^{\prime}(x)\right\|}
$$


O campo $\mu=h_{n}^{*}(\mu)$ é invariante por $P_{c}^{n}$, pois

$$
\begin{aligned}
\left(P_{c}^{n}\right)^{*} h_{n}^{*}(\mu) & =\left(h_{n} \circ P_{c}^{n}\right)^{*}(\mu) \\
& =\mu\left(h_{n} \circ P_{c}^{n}\right) \frac{\overline{h_{n}^{\prime}\left(P_{c}^{n}\right)}}{h_{n}^{\prime}\left(P_{c}^{n}\right)} \frac{\left(P_{c}^{n}\right)^{\prime}}{\left(P_{c}^{n}\right)^{\prime}} \\
& =\mu\left(P_{c}^{n} \circ h_{n}\right) \frac{\overline{\left(P_{c}^{n}\right)^{\prime}}}{\left(P_{c}^{n}\right)^{\prime}} \frac{h_{n}^{\prime}\left(P_{c}^{n}\right)}{h_{n}^{\prime}\left(P_{c}^{n}\right)} \\
& =h_{n}^{*}\left(P_{c}^{n}\right)^{*}(\mu) \\
& =h_{n}^{*}(\mu)
\end{aligned}
$$

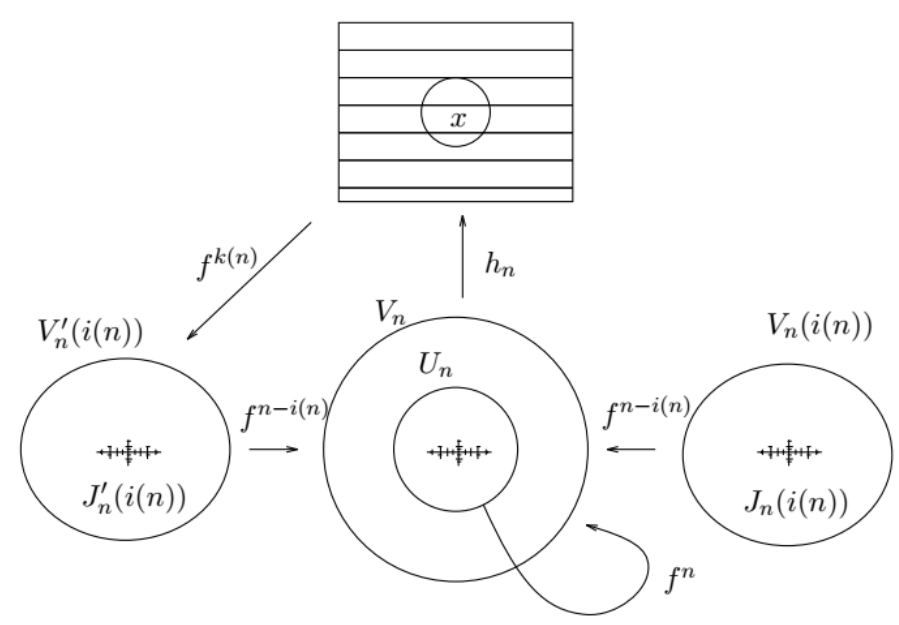

Figura 5.1: Construção do campo de linhas univalente $\nu$

Prosseguimos definindo a aplicação $A_{n}(z)=\frac{z}{\operatorname{diâm}_{\mathbb{E}}\left(J_{n}\right)}$ para $z \in \mathbb{C}$ e consideramos as aplicações $g_{n}=A_{n} \circ P_{c}^{n} \circ A_{n}^{-1}:\left(A_{n}\left(U_{n}\right), 0\right) \longrightarrow\left(A_{n}\left(V_{n}\right), A_{n}\left(P_{c}^{n}(0)\right)\right)$ que são do tipo quadrático cujo conjunto de Julia tem diâmetro euclideano igual a 1 e $\bmod \left(A_{n}\left(U_{n}\right), A_{n}\left(V_{n}\right)\right) \geq m$.

Já que $h_{n}^{-1}(x) \in J_{n}$, temos $y_{n} \in J\left(g_{n}\right)$ em que $y_{n}:=A_{n}\left(h_{n}^{-1}(x)\right)$.

Isto posto, concluímos a partir do teorema 5.8-[McM1] a existência de uma subsequência de níveis $n \in \operatorname{USR}(c, m)$ tal que $g_{n}$ converge na topologia de Carathéodory a uma aplicação do tipo polinomial de grau 2

$$
g:(U, 0) \longrightarrow(V, g(0))
$$

com $\bmod (U, V) \geq m$.

Seja $k_{n}:=h_{n} \circ A_{n}^{-1}: A_{n}\left(V_{n}\right) \stackrel{A_{n}^{-1}}{\longrightarrow} V_{n} \stackrel{h_{n}}{\longrightarrow} \mathbb{C}$.

Desta forma, $k_{n}\left(y_{n}\right)=x$ e o campo de linhas $\nu_{n}:=k_{n}^{*}(\mu)$ em $A_{n}\left(V_{n}\right)$ é invariante por $g_{n}$, pois

$$
\begin{aligned}
\left(g_{n}\right)^{*}\left(k_{n}^{*}(\mu)\right) & =\left(k_{n} \circ g_{n}\right)^{*}(\mu) \\
& =\left(h_{n} \circ P_{c}^{n} \circ A_{n}^{-1}\right)(\mu) \\
& =\left(A_{n}^{-1}\right)^{*}\left(P_{c}^{n}\right)^{*} h_{n}^{*}(\mu) \\
& =\left(A_{n}^{-1}\right)^{*} h_{n}^{*}(\mu) \\
& =k_{n}^{*}(\mu)
\end{aligned}
$$


Mostraremos a seguir que $\left|k_{n}^{\prime}\left(y_{n}\right)\right| \rightarrow 0$. Jenkins em [Jen] obteve a seguinte desigualdade válida para qualquer função $f$ holomorfa univalente no disco unitário $\mathbb{D}$ e qualquer $p \geq 1$

$$
\left|f\left(z_{2}\right)-f\left(z_{2}\right)\right| \geq \frac{\sinh (2 \rho)}{2(2 \cosh (2 \rho p))^{1 / p}}\left(\left|D_{1} f^{\prime}\left(z_{1}\right)\right|^{p}+\left|D_{1} f^{\prime}\left(z_{2}\right)\right|^{p}\right)^{1 / p}
$$

em que $\rho$ é a distância entre $z_{1}$ e $z_{2}$ na métrica de Poincaré e $D_{1} f^{\prime}(z):=\left(1-|z|^{2}\right) f^{\prime}(z)$.

Como $x \notin \mathrm{P}(c)$ e $\mathrm{P}(c)$ é fechado temos $d_{\mathbb{H}}(x, \mathrm{P}(c)):=r>0$, em particular, $d_{\mathbb{D}}(x, 0):=r>0$. Logo, $\mathrm{d}_{\mathbb{D}}\left(y_{n}, 0\right):=s>0$ para todo $n \geq 1$, pois $k_{n}\left(y_{n}\right)=x$.

Assim, no intuito de fazermos uso desta estimativa, tomaremos uma sequência de aplicações de Möebius $\left\{M_{n}\right\}_{n \in \mathbb{N}}$ que matenha o 0 fixado e mapeie um ponto $w \neq 0$ em $y_{n}$ de modo que a derivada de $M_{n}$ em $w$ fique limitada inferiormente uniformemente acima de 0. Podemos tomar tal sequência devido $\inf _{n}\left\{\left|y_{n}\right| ; n \geq 1\right\}>0$, assim nos basta tomar $M_{n}(z):=\frac{y_{n} \cdot z}{2 \operatorname{diâm}_{\mathbb{E}}\left(J\left(g_{n}\right)\right) w}$.

Isto posto, recorrendo à 5.26 tomando $p=1$, resulta

$$
\left|k_{n}\left(y_{n}\right)-k_{n}(0)\right| \geq C_{0}\left(C_{1}\left|k_{n}^{\prime}(0)\right|+C_{2}\left|k_{n}^{\prime}\left(y_{n}\right)\right|\right)
$$

em que $C_{0}:=\frac{\sinh (2 \rho)}{2(2 \cosh (2 \rho))} ; C_{1}:=\left|M_{n}^{\prime}(0)\right|$ e $C_{2}:=\left|\left(1-|w|^{2}\right)\right|\left|M_{n}^{\prime}(w)\right|$. No entanto, $\operatorname{diâm}_{\mathbb{H}}\left(k_{n}\left(J\left(g_{n}\right)\right)\right)=$ $\operatorname{diâm}_{\mathbb{H}}\left(J_{n}^{*}\right) \rightarrow 0$ quando $n \rightarrow+\infty$. Portanto, $\left|k_{n}^{\prime}\left(y_{n}\right)\right| \rightarrow 0$.

Como $y_{n} \in J\left(g_{n}\right)$ e $J\left(g_{n}\right)$ fica contido na componente compacta do complementar em $\mathbb{C}$ de um anel em $A_{n}\left(V_{n}\right)$ com módulo definido, do teorema 5.3-[McM1], passando a uma subsequência temos $\left(A_{n}\left(V_{n}\right), y_{n}\right) \rightarrow(V, y)$.

Já que $\left|x-k_{n}\left(y_{n}\right)\right|=0$, então, $\sup \frac{\left|x-k_{n}\left(y_{n}\right)\right|}{\left|k_{n}^{\prime}\left(y_{n}\right)\right|}=0$, logo, pelo teorema 5.16-[McM1] passando, se necessário, a uma subsequência de níveis $n \in \operatorname{USR}(c, m)$ da sequência no passo anterior, o campo de linhas $\nu_{n}:=k_{n}^{*}(\mu)$ convergirá em medida a um campo de linhas univalente $\nu$ em $V$. Mas, $\nu_{n}$ é invariante por $g_{n}$ e, portanto, o teorema 5.14-[McM1] nos assegura que $\nu$ é invariante por $g$.

Agora, pela definição da topologia de Carathéodory $g_{n}$ converge uniformemente nas partes compactas de $(U, 0)$ para $g$. Isto posto, pelo teorema da convergência de Weierstrass $(\S 4-[\mathrm{R}])$, $g_{n}^{\prime} \rightarrow g^{\prime}$ converge uniformemente nas partes compactas de $(U, 0)$, em particular, $g_{n}^{\prime}(0) \rightarrow g^{\prime}(0)$, mas, $g_{n}^{\prime}(0)=\left(P_{c}^{n}\right)^{\prime}(0)=0$, então, $g^{\prime}(0)=0$. E como $0 \in V$ decorre do teorema 5.13-[McM1] que $g$ não admite um campo de linhas invariante univalente.

Em conclusão, $P_{c}$ não pode ter um campo de linhas suportado em seu conjunto de Julia.

Obteremos o teorema de rigidez 5.1 a partir do teorema 5.5 para o caso no qual $\lim \inf _{n \in \mathrm{SR}^{*}(c)} l_{\mathbb{H}}\left(\gamma_{n}\right)$ é suficientemente pequeno. Para tanto, relacionaremos o comprimento da geodésica $\gamma_{n}$ com a existência de uma renormalização regular com módulo definido(i.e. $\bmod \left(U_{n}, V_{n}\right) \geq m>0$ em que $\left(P_{c}^{n}, U_{n}, V_{n}\right)$ é uma renormalização simples regular).

Teorema 5.6. Suponhamos $P_{c}$ infinitamente renormalizável e $P_{c}^{n}$ simplesmente renormalizável. Então, podemos tomar $U_{n}$ e $V_{n}$ discos topológicos tais que $P_{c}^{n}: U_{n} \longrightarrow V_{n}$ seja uma renormalização regular. E quando $l_{\mathbb{H}}\left(\gamma_{n}\right)$ é suficientemente pequeno, garantimos ainda

$$
\bmod \left(U_{n}, V_{n}\right)>M\left(l_{\mathbb{H}}\left(\gamma_{n}\right)\right)>0
$$

em que $M(l) \rightarrow+\infty$ quando $l \rightarrow 0$. 
Demonstração. Dada uma renormalização $P_{c}^{n}: \widetilde{U_{n}} \longrightarrow \widetilde{V_{n}}$ podemos substituir $\widetilde{V_{n}}$ por um disco $V_{n}$ de modo que $\partial V_{n}$ fique arbitrariamente próximo de $K_{n}$ tomando a componente de $P_{c}^{-d n}\left(\widetilde{V_{n}}\right)$ que contem 0 para um $d>0$ suficientemente grande. Dessa maneira, conseguimos fazer $V_{n}$ disjunto de $\mathrm{P}(c)-P_{n}$, pois, sendo os pequenos conjuntos pós-críticos $P_{n}(i)$ disjuntos, $\mathrm{P}(c)-P_{n}=\mathrm{P}(c)-K_{n}$ é compacto.

Mostraremos agora que se o $l_{\mathbb{H}}(l)$ é suficientemente pequeno, isto é, se a geodésica $\gamma_{n}$ em $\widehat{\mathbb{C}}-\mathrm{P}(c)$ é curta, então, $P_{c}^{n}$ possue uma renormalização regular $\left(P_{c}^{n}, U_{n}, V_{n}\right)$ para a qual $\bmod \left(U_{n}, V_{n}\right)$ é grande.

Seja $A_{n}$ a vizinhança colar de $\gamma_{n}$ com respeito à métrica hiperbólica em $\widehat{\mathbb{C}}-\mathrm{P}(c)$. Provém do teorema 2.19-[McM1] que o módulo de $A_{n}$ é grande quando o comprimento hiperbólico de $\gamma_{n}$ é pequeno em $\widehat{\mathbb{C}}-\mathrm{P}(c)$.

Cosideremos $B_{n}$, o anel em $P_{c}^{-n}\left(A_{n}\right) \subset \widehat{\mathbb{C}}-\mathrm{P}(c)$ que contém a componente da multicurva $P_{c}^{-n}\left(\gamma_{n}\right)$ que é homotópica à $\gamma_{n}$ em $\widehat{\mathbb{C}}-\mathrm{P}(c)$. Em seguida, tomemos os discos $D_{n}$ e $E_{n}$ obtidos de $A_{n}$ e $B_{n}$, respectivamente, os unido com a componente de seu complementar que contém o 0 . Note que $0 \notin P_{c}^{i}\left(E_{n}\right)$ para $i \in\{1,2, \cdots, n-1\}$. Isto posto, $P_{c}^{n}: E_{n} \longrightarrow D_{n}$ é uma aplicação própria de grau igual a 2, pois $P_{c}^{n}\left(\partial E_{n}\right)=\partial D_{n}$. Temos também, $E_{n} \cap \mathrm{P}(c)=D_{n} \cap \mathrm{P}(c)=P_{n}(n)$, pois $K_{n} \subset E_{n} \subset D_{n}$; e, portanto, $P_{c}^{n}: E_{n} \longrightarrow D_{n}$ é critiamente compacta.

Por ser $P_{c}$ infinitamente renormalizável ele não possue cíclo atrator, logo, do teorema 5.12-[McM1] se conclue que $P_{c}^{n}$ admite uma renormalização $P_{c}^{n}: U_{n} \longrightarrow V_{n} \operatorname{com} U_{n} \subset V_{n}$ quando $\bmod \left(P_{n}(n), E_{n}\right)$ for suficientemente grande, isto é, $\bmod \left(P_{n}(n), E_{n}\right)>M_{2}$ (consulte 5.12-[McM1]).

Fazendo o pullback por $P_{c}^{n}$ do anel $V_{n}-\overline{U_{n}}$ um número $d$ de vezes suficiente, o pullback deste anel, $\widetilde{V_{n}}-\widetilde{\widetilde{U}_{n}}$, fica contido em $D_{n}$. E desta maneira, $P_{c}^{n}: \widetilde{U_{n}} \longrightarrow \widetilde{V_{n}}$ é uma renormalização de $P_{c}^{n}$ tal que $\widetilde{U_{n}} \subset E_{n}$ e $\widetilde{V_{n}} \subset D_{n}$. Neste caso teremos,

$$
\frac{\bmod \left(\widetilde{V_{n}}-\widetilde{U_{n}}\right)}{2^{d}}=\bmod \left(V_{n}-\overline{U_{n}}\right)
$$

Mas, do teorema 5.12-[McM1], temos $\bmod \left(V_{n}-\overline{U_{n}}\right)>m_{2}\left(\bmod \left(P_{n}(n), D_{n}\right)\right) . \operatorname{Assim}, \bmod \left(V_{n}-\right.$ $\left.\overline{U_{n}}\right)$ é limitado inferiormente em termos de $l_{\mathbb{H}}\left(\gamma_{n}\right), \operatorname{pois}, \bmod \left(P_{n}(n), D_{n}\right)>\bmod \left(A_{n}\right)=M\left(l_{\mathbb{H}}\left(\gamma_{n}\right)\right)$. E desde que, o módulo da vizinhança colar depende apenas do comprimento hiperbólico de $\gamma_{n}$ em $\widehat{\mathbb{C}}-\mathrm{P}(c)$ e tende continuamente para o infinito quando o comprimento tende para zero, temos o que queríamos(consulte 2.19-[McM1]).

Note ainda que tal renormalização é regular, devido ao teorema da vizinhaça colar, do qual decorre $A_{n} \cap \mathrm{P}(c)=\varnothing$.

Corolário 5.2 (rigidez). Existe uma constante $L>0$ tal que se

$$
\liminf _{\mathrm{SR}^{*}} l_{\mathbb{H}}\left(\gamma_{n}\right)<L,
$$

então, $P_{c}$ não suporta um campo de linhas invariante em seu Julia.

Demonstração. Pelo teorema 5.6 podemos tomar $L>0$ pequeno o suficiente tal que de $l_{\mathbb{H}}\left(\gamma_{n}\right)<L$ decorra que $P_{c}^{n}$ admite uma renormalização regular e $\bmod \left(U_{n}, V_{n}\right)>m>0 \operatorname{com} m=m(L)$ fixado.

Logo, do teorema 5.5, concluímos o enunciado. 
Em particular, o resulto se aplica para o caso em que $L=0$. e temos portanto a primera parte do teorema.

Geometricamente, neste caso, a superfície de Riemann $\widehat{\mathbb{C}}-\mathrm{P}(c)$ possue indinitas partes que são bem delgadas.

\subsection{Segunda Parte}

Nesta subseção provamos o teorema de rigidez para o caso em que $0<\lim \inf _{\mathrm{SR}^{*}(c)}<+\infty$. Isto é, provaremos o seguinte:

Teorema 5.7. Suponhamos $P_{c}$ infinitamente renormalizável e tal que $0<\liminf _{S R^{*}(c)}<+\infty$. Então, $J(c)$ não suporta um campo de linhas invariante.

Fixemos inicialmente algumas definições e notações.

Definição 5.3. Para $n \in S R^{*}(c)$,

1. $\delta_{n}$ denotará a única componente da multicurva $P_{c}^{-n}\left(\gamma_{n}\right)$ que é isotópica à $\gamma_{n}$ em $\widehat{\mathbb{C}}-P(c)$;

2. $X_{n}$ denotará o disco em $\mathbb{C}$ cuja fronteira é a curva $\delta_{n}$;

3. $Y_{n}$ denotará o disco cuja fronteira é a curva $\gamma_{n}$.

Com isso, $P_{c}^{n}: X_{n} \longrightarrow Y_{n}$ será uma aplicação própria de grau igual a 2.;

4. $Y_{n}(i):=P_{c}^{i}\left(X_{n}\right)$ para cada $i \in\{1,2, \cdots, n\}$. Desta forma, $\left.P_{c}^{i}\right|_{X_{n}}$ se fatora como segue

$$
X_{n} \stackrel{P_{c}}{\longmapsto} Y_{n}(1) \stackrel{P_{c}}{\longmapsto} Y_{n}(2) \cdots \stackrel{P_{c}}{\longmapsto} Y_{n}(n-1) \stackrel{P_{c}}{\longmapsto} Y_{n}(n):=Y_{n}
$$

na qual $X_{n} \stackrel{P_{c}}{\longmapsto} Y_{n}(1)$ tem grau igual a 2 e as demais são univalentes. Note ainda que $Y_{n}(i) \cap$ $\mathrm{P}(c)=P_{n}(i)$, pois $\delta_{n} \approx_{i s o t} \gamma_{n}$;

5. $y_{n}:=\bigcup_{i=1}^{n} Y_{n}(i)$ e, então, $P(c) \subset \mathcal{J}_{n}$;

6. $Y_{n}^{\prime}(i)=-Y_{n}(i)$ para cada $i \in\{1,2, \cdots, n-1\}$. Como $Y_{n}(i) \stackrel{P_{c}}{\longmapsto} Y_{n}(i+1)$ é univalente e $P_{c}(-z)=P_{c}(z), Y_{n}^{\prime}(i) \stackrel{P_{c}}{\longmapsto} Y_{n}(i+1)$ é também univalente e, portanto, $Y_{n}(i) \cap Y_{n}^{\prime}(i)=\varnothing$. Em particula, $Y_{n}^{\prime}(i)$ é disjunto do conjunto pós-crítico $\mathrm{P}(c)$.

Definição 5.4. Ponhamos $\operatorname{SR}(c, \lambda):=\left\{n \in \mathrm{SR}^{*}(c) ; \lambda^{-1}<l_{\widehat{\mathbb{C}}-(\mathrm{P}(c))}\left(\gamma_{n}\right)<\lambda\right\}$ para $\lambda \in(0,+\infty)$.

Sob a hipótese, $0<\lim \inf _{\mathrm{SR}^{*}} l(\gamma)<+\infty$, é certo que existe $\lambda \in(0,+\infty)$ tal que $\operatorname{SR}(c, \lambda)$ seja infinito.

Teorema 5.8. Se $n \in \mathrm{SR}(c, \lambda)$, então, com respeito à métrica euclidiana, temos

$$
\begin{aligned}
\operatorname{diâm}_{\mathbb{E}}\left(X_{n}\right) & \geq \operatorname{diâm}_{\mathbb{E}}\left(B_{n}\right) \geq C(\lambda) \operatorname{diâm}_{\mathbb{E}}\left(X_{n}\right) \\
\operatorname{diâm}_{\mathbb{E}}\left(Y_{n}\right) & \geq \operatorname{diâm}_{\mathbb{E}}\left(B_{n}\right) \geq C(\lambda) \operatorname{diâm}_{\mathbb{E}}\left(Y_{n}\right)
\end{aligned}
$$

em que $B_{n}$ é a maior bola euclidiana centrada na origem, $z=0$, contida em $X_{n} \cap Y_{n}$. 
Para uma prova consulte o teorema 10.12-[McM1].

De maneira análoga temos também,

$$
\begin{aligned}
& \operatorname{diâm}_{\mathbb{E}}\left(Y_{n}(i)\right) \geq \operatorname{diâm}_{\mathbb{E}}\left(B_{n}(i)\right) \geq C(\lambda) \operatorname{diâm}_{\mathbb{E}}\left(Y_{n}(i)\right) \\
& \operatorname{diâm}_{\mathbb{E}}\left(\widetilde{Y}_{n}(i)\right) \geq \operatorname{diâm}_{\mathbb{E}}\left(B_{n}(i)\right) \geq C(\lambda) \operatorname{diâm}_{\mathbb{E}}\left(\widetilde{Y}_{n}(i)\right)
\end{aligned}
$$

em que $B_{n}(i)$ é a maior bola euclidiana centrada $P_{c}^{i}(0)$, contida em $Y_{n}(i) \cap \widetilde{Y}_{n}(i)$.

Teorema 5.9. Para $P_{c}$ robusto temos

$$
P(c) \cap \mathbb{C}=\bigcap_{S R(c)} y_{n}
$$

Demonstração. Pelo teorema 4.4, $\operatorname{diâm}\left(P_{n}(i)\right) \rightarrow 0$ quando $n \rightarrow+\infty$ em SR(c). Uma vez que $P_{n}(i) \subset Y_{n}(i)$ é suficiente mostrar que que $\sup _{i} \operatorname{diâm}_{\mathbb{H}}\left(Y_{n}(i)\right) \rightarrow 0$, e assim como na demosntração do teorema 5.4 obteremos a igualdade enunciada. Note que $\left\{y_{n}\right\}_{n \in \operatorname{SR}(c)}$ é uma sequência encaixada decrescente. Mas pelo teorema 5.8 mais as desigualdades 5.34 e 5.35 , mostrar que que $\sup _{i} \operatorname{diâm}_{\mathbb{H}}\left(Y_{n}(i)\right) \rightarrow 0$ é o mesmo que mostrar que $\sup _{i} \operatorname{diâm}_{\mathbb{H}}\left(\widetilde{Y}_{n}(i)\right) \rightarrow 0$. O que, agora, é imediato já que cada $\widetilde{Y}_{n}(i)$ fica contido em alguma componente de $D_{m}$ para $m<n$ em $\operatorname{SR}(c, \lambda)$ onde $D_{m}$ são os mesmos conjuntos definidos na demonstração do teorema 4.4 para os quais tínhamos o diâmetro euclidiano decrescendo para zero.

Corolário 5.3. Se $P_{c}$ é robusto, então, para quase todo ponto $z \in J(c)$ valem as seguintes propriedades

1. para qualquer $n \in \mathrm{SR}^{*}(c)$ existe um inteiro $k>0$ tal que $P_{c}^{k}(z) \in \mathrm{y}_{n}$;

2. para qualquer inteiro $k>0$ existe $n \in \mathrm{SR}^{*}(c)$ tal que $P_{c}^{k}(z)$ não pertence ao $y_{n}$.

Teorema 5.10. Suponhamos $|S R(c, \lambda)|=\infty$ e definamos a aplicação afim $A_{n}(z):=\frac{z}{\operatorname{diâm}_{\mathbb{E}}\left(B_{n}\right)}$. Então, na topologia de Carathéodory, existe uma subsequência de niveis $n \in S R(c, \lambda)$ tal que

$$
\begin{aligned}
\left(A_{n}\left(X_{n}\right), 0\right) & \longrightarrow(X, 0) ; \\
\left(A_{n}\left(Y_{n}\right), P_{c}^{n}(0)\right) & \longrightarrow(Y, P(0)) \quad \mathrm{e} \\
A_{n} \circ P_{c}^{n} \circ A_{n}^{-1} & \longrightarrow P
\end{aligned}
$$

em que $P:(X, 0) \longrightarrow(Y, P(0))$ é uma aplicação própria de grau 2 com $0 \in X \cap Y$ e $P^{\prime}(0)=0$.

Demonstração. Da definição de $A_{n}$ temos $A_{n}\left(B_{n}\right)=B(0,1 / 2)$, mas $B_{n} \subset X_{n} \cap Y_{n}$ e, portanto, tanto $A_{n}\left(x_{n}\right)$ quanto $A_{n}\left(Y_{n}\right)$ contém $B(0,1 / 2)$ para qualquer $n \in \operatorname{SR}(c, \lambda)$.

Com isso, do teorema 5.2-[McM1], passando a auma subsequência se necessário for, temos

$$
\begin{aligned}
\left(A_{n}\left(X_{n}\right), 0\right) & \rightarrow(X, 0) \\
\left(A_{n}\left(Y_{n}\right), 0\right) & \rightarrow(Y, 0)
\end{aligned}
$$

quando $n \rightarrow+\infty$ em $\operatorname{SR}(c, \lambda)$. 
Recorrendo ao teorema da vizinhança colar 2.18-[McM1], consideremos o anel $C\left(\gamma_{n}\right)$. Como $l\left(\gamma_{n}\right) \leq \lambda$ para todo $n \in \mathrm{SR}(c, \lambda)$, pelo teorema 2.19 e 2.4-[McM1], o diâmetro hiperbólico em $\widehat{\mathbb{C}}-\mathrm{P}(c)$ de $Y_{n}$ é limitado superiormente por uma constante $C(\lambda)$ para todo $n \in \mathrm{SR}(c, \lambda)$, logo temos o mesmo para o diâmetro hiperbólico de $P_{n}(n)$, pois $P_{n}(n) \subset Y_{n}$.

Disto decorre que $\mathrm{d}_{\mathbb{H}}\left(0, P_{c}^{n}(0)\right)$ é limitado, já que $P_{c}^{n}(0) \in P_{n}(n)$. Isto posto, do teorema $5.3-[\mathrm{McM} 1]$, tomando uma subsequência se necessário, seguirá que $\left(A_{n}\left(Y_{n}\right), P_{c}^{n}(0)\right) \rightarrow(Y, P(0))$, em que $P(0)=\lim _{n \rightarrow+\infty ; n \in \mathrm{SR}^{*}} P_{c}^{n}(0)$.

Do teorema 10.13 temos

$$
\begin{aligned}
\operatorname{diâm}_{\mathbb{E}}\left(X_{n}\right) & \leq \frac{\operatorname{diâm}_{\mathbb{E}}\left(B_{n}\right)}{C(\lambda)} \\
\operatorname{diâm}_{\mathbb{E}}\left(Y_{n}\right) & \leq \frac{\operatorname{diâm}_{\mathbb{E}}\left(B_{n}\right)}{C(\lambda)}
\end{aligned}
$$

Logo, sendo $A_{n}$ afim,

$$
\operatorname{diâm}_{\mathbb{E}}\left(A_{n}\left(X_{n}\right)\right)=\frac{\operatorname{diâm}_{\mathbb{E}}\left(X_{n}\right)}{\operatorname{diâm}_{\mathbb{E}}\left(B_{n}\right)}
$$

Analogamente, vale

$$
\operatorname{diâm}_{\mathbb{E}}\left(A_{n}\left(Y_{n}\right)\right)=\frac{\operatorname{diâm}_{\mathbb{E}}\left(Y_{n}\right)}{\operatorname{diâm}_{\mathbb{E}}\left(B_{n}\right)}
$$

A partir disto segue

$$
\begin{aligned}
\operatorname{diâm}_{\mathbb{E}}\left(A_{n}\left(X_{n}\right)\right) & \leq \frac{1}{C(\lambda)} \\
\operatorname{diâm}_{\mathbb{E}}\left(A_{n}\left(Y_{n}\right)\right) & \leq \frac{1}{C(\lambda)}
\end{aligned}
$$

Desta forma, $X$ e $Y$ não são iguais a $\mathbb{C}$, e como consequência disso, estamos áptos para aplicar o teorema 5.6-[McM1]. Assim, $A_{n} \circ P_{c}^{n} \circ A_{n}^{-1}$ converge para uma aplicação própia $P: X \longrightarrow Y$ de grau menor ou igual à 2 . No entanto, $g_{n}^{\prime}(0)=\left(P_{c}^{n}\right)^{\prime}(0)=0$, logo pelo teorema da convergênicia de Weierstrass $(\S 4-[\mathrm{R}]), P^{\prime}(0)=0$. Portanto, $P: X \longrightarrow Y$ é aplicação própia de grau 2.

Teorema 5.11. Para qualquer $n \in \mathrm{SR}^{*}(c)$,

$$
\begin{aligned}
l_{\mathbb{H}}\left(\gamma_{n}\right) & \leq l_{\mathbb{H}}\left(\delta_{n}\right) \leq 2 l_{\mathbb{H}}\left(\gamma_{n}\right) \mathrm{e} \\
l_{\mathbb{H}}\left(\gamma_{n}(i)\right) & \leq l_{\mathbb{H}}\left(\partial Y_{n}(i)\right) \leq l_{\mathbb{H}}\left(\gamma_{n}\right)
\end{aligned}
$$

na métrica hiperbólica em $\widehat{\mathbb{C}}-P(c)$.

Demonstração. $\partial Y_{n}(i)=P_{c}^{i}\left(\delta_{n}\right)$ e é isotópica à $\gamma_{n}(i)$, pois $P_{n}(i) \subset \operatorname{int}_{\mathbb{C}}\left(\partial Y_{n}(i)\right)$, em que $i_{\mathbb{C}} t_{\mathbb{C}}\left(\partial Y_{n}(i)\right)$ é o interior de $\partial Y_{n}(i)$ em $\mathbb{C}$.

As desigualdades à esquerda decorrem do fato de que $\gamma_{n}$ e $\gamma_{n}(i)$ são os representantes geodésicos das classes de homotopia livre de $\delta_{n}$ e $\partial Y_{n}(i)$ respectivamente.

Para obtermos as desigualdades à direita notemos que $P_{c}^{n}$ mapeia $\delta_{n}$ sobre $\gamma_{n}$ com grau igual à $2, P_{c}^{n-i}$ mapeia $\partial Y_{n}(i)$ sobre $\gamma_{n}$ com grau 1. A partir disto e do teorema 3.5-[McM1] obtemos tais 
desigualddades, pois na métrica hiperbólica de $\widehat{\mathbb{C}}-\mathrm{P}(c)$

$$
\begin{aligned}
2 l_{\mathbb{H}}\left(\gamma_{n}\right) & =2 \int_{\gamma_{n}} \rho(z)|d z|=\int_{\delta_{n}}\left\|\left(P_{c}^{n}\right)^{\prime}(z)\right\| \rho(z)|d z| \\
& \geq \int_{\delta_{n}} \rho(z)|d z|=l_{\mathbb{H}}\left(\delta_{n}\right)
\end{aligned}
$$

De modo similar, temos

$$
l_{\mathbb{H}}\left(\gamma_{n}\right) \geq l_{\mathbb{H}}\left(Y_{n}(i)\right)
$$

Corolário 5.4. Para cada $n \in \mathrm{SR}(c, \lambda), \delta_{n}$ fica contida em uma $C(\lambda)$-vizinhança de $\gamma_{n}$ e $\partial Y_{n}(i)$ fica contida em uma $C(\lambda)$-vizinhança de $\gamma_{n}(i)$.

Demonstração. Pelo precedente teorema e como $\lambda^{-1} \leq l_{\mathbb{H}}\left(\gamma_{n}\right)$ aplicando-se o teorema 2.23-[McM1] obtemos uma cota superior $C(\lambda)$ para a distância de qualquer ponto $x \in \delta_{n}$ à $\gamma_{n}$.

Valendo-se do teorema 4.3 temos $l_{\mathbb{H}}\left(\gamma_{n}(i)\right) \leq \frac{1}{2 \lambda}$. Assim, do teorema precedente e o 2.23-[McM1] obtemos uma cota superior $C(\lambda)$ para a distância de qualquer ponto $x \in Y_{n}(i)$ à $\gamma_{n}$.

Teorema 5.12. Para qualquer $n \in \operatorname{SR}(c, \lambda)$

$$
\begin{aligned}
\left\|\left(P_{c}^{n}\right)^{\prime}(x)\right\| & \leq C(\lambda) \quad \text { para } x \in \delta_{n}=\partial X_{n} e \\
\left\|\left(P_{c}^{n-i}\right)^{\prime}(x)\right\| & \leq C(\lambda) \quad \text { para } x \in \partial Y_{n}(i)
\end{aligned}
$$

com respeito à métrica hiperbólica em $\widehat{\mathbb{C}}-\mathrm{P}(c)$.

Demonstração. Temos,

$$
2 l_{\mathbb{H}}\left(\gamma_{n}\right)=\int_{\delta_{n}}\left\|\left(P_{c}^{n}\right)^{\prime}(z)\right\| \rho(z)|d z|
$$

e $\left\|\left(P_{c}^{n}\right)^{\prime}\left(z_{1}\right)\right\| \leq 2$ para algum $z_{1} \in \delta_{n}$, pois caso contrário

$$
2 l_{\mathbb{H}}\left(\gamma_{n}\right)=\int_{\delta_{n}}\left\|\left(P_{c}^{n}\right)^{\prime}(z)\right\| \rho(z)|d z|>2 \int_{\delta_{n}} \rho(z)|d z|=2 l_{\mathbb{H}}\left(\delta_{n}\right)
$$

contradizendo que $l_{\mathbb{H}}\left(\gamma_{n}\right) \leq l_{\mathbb{H}}\left(\delta_{n}\right)$.

Qualquer ponto $z_{2} \in \delta_{n}$ está conectado à $z_{1}$ por um arco $\eta$ de $\delta_{n}$ tal que $P_{c}^{n}(\eta)$ é um subarco de $\gamma_{n}$ e, portanto, $l_{\mathbb{H}}\left(P_{c}^{n}(\eta)\right) \leq l_{\mathbb{H}}\left(\gamma_{n}\right) \leq \lambda$.

Consequentemente, advém do teorema 3.8 quem

$$
\left\|\left(P_{c}^{n}\right)^{\prime}\left(z_{2}\right)\right\| \leq\left\|\left(P_{c}^{n}\right)^{\prime}\left(z_{1}\right)\right\| \leq 2^{\alpha}
$$

em que $\alpha=\exp \left(C l_{\mathbb{H}}\left(P_{c}^{n}(\eta)\right)\right) \leq \exp (C \lambda)$.

Portanto, $\left\|\left(P_{c}^{n}\right)^{\prime}(z)\right\|$ é linitado superiormente por $C(\lambda)=2^{\exp (C \lambda)}$.

Mas, lembre-se que $\partial Y_{n}(i)=P_{c}^{i}\left(\delta_{n}\right)$, deste modo, cada $z \in \partial Y_{n}(i)$ é tal que $z=P_{c}^{i}(w)$ para 
algum $w \in \delta_{n}$. Notemos o seguinte

$$
\left(P_{c}^{n-i}\right)^{\prime}(z)=\prod_{k=1}^{n-i} P_{c}^{\prime}\left(P_{c}^{n-k}(z)\right)
$$

Mas, do teorema 3.5-[McM1] segue

$$
\left\|\left(P_{c}^{n-i}\right)^{\prime}(z)\right\| \leq \prod_{k=1}^{n} P_{c}^{\prime}\left(P_{c}^{n-k}(z)\right)=\left\|\left(P_{c}^{n}\right)^{\prime}(z)\right\| \leq 2^{\alpha} .
$$

Teorema 5.13. Para qualquer $n \in \mathrm{SR}(c, \lambda)$, existe $i \neq j$ tais que

$$
\mathrm{d}\left(\gamma_{n}(i), \gamma_{n}(j)\right)<C(\lambda)
$$

na métrica hiperbólica de $\widehat{\mathbb{C}}-\mathrm{P}(c)$.

Demonstração. Fixado um nível qualquer $n \in \mathrm{SR}(c, \lambda)$, consideremos a superfície de Riemann de tipo finito $X$ obtida retirando-se de $\mathbb{C}$ todos os discos limitados pelas curvas $\gamma_{n}(i)$ para $i \in$ $\{1,2, \cdots, n\}$. Desta maneira $X$ tem fronteira geodésica $\cup_{i=1}^{n} \gamma_{n}(i)$ e uma cúspide em $\infty$. Mas, como pelo teorema $4.3, l_{\widehat{\mathbb{C}}-P(c)}\left(\gamma_{n}(i)\right) \geq(2 \lambda)^{-1}$. E com isso o teorema 2.24-[McM1] nos garante a existência de índices $i$ e $j$ diferentes tais que $\mathrm{d}\left(\gamma_{n}(i), \gamma_{n}(j)\right)<C(\lambda)$.

Teorema 5.14. Para $n \in S R(c, \lambda)$ existe um disco $Z_{n} \subset \widehat{\mathbb{C}}-\mathrm{P}(c)$ e um inteiro $m$ com $0<m<n$ tal que

1. $P_{c}^{m}: Z_{n} \longrightarrow Y_{n}$ é univalente;

2. $\mathrm{d}_{\mathbb{H}}\left(\partial X_{n}, \partial Y_{n}\right)<C(\lambda)$;

3. $l_{\mathbb{H}}\left(\partial Z_{n}\right)<\lambda$;

4. área $\left(Z_{n}\right)>(C(\lambda))^{-1}$.

com respeito a métrica hiperbólica em $\widehat{\mathbb{C}}-\mathrm{P}(c)$.

Demonstração. Vimos logo acima que fixado um nível $n \in \operatorname{SR}(c, \lambda)$ existem $\gamma_{n}(i)$ e $\gamma_{n}(j)$ diferentes tais que $\mathrm{d}\left(\gamma_{n}(i), \gamma_{n}(j)\right)$ é limitada em termos de $\lambda$. Já o corolário 5.4 nos garante que $\partial Y_{n}(i)$ fica uniformemente próximo de $\gamma_{n}(i)$ e assim, $\mathrm{d}\left(\partial Y_{n}(i), \partial Y_{n}(j)\right)<C(\lambda)$.

Considere $\alpha$ uma geodésica de comprimento menor do que $C(\lambda)$. Se $\partial Y_{n}(i)$ e $\partial Y_{n}(i)$ tem intersecção não vazia, então, "tomamos" $\alpha$ com comprimento zero.

Suponhamos $i<j$.

A aplicação $P_{c}^{i-1}: Y_{n}(1) \longrightarrow Y_{n}(i)$ é univalente, logo admite uma inversa univalente, $P_{c}^{1-i}$ : $Y_{n}(i) \longrightarrow Y_{n}(1)$.

Como $\left(\alpha \cup Y_{n}(j)\right) \cap \mathrm{P}(c)=P_{n}(j)$, podemos obter uma continuação analítica deste ramo de $\left.P_{c}^{1-i}\right|_{Y_{n}(i)}$ ao longo de $\alpha$ à uma aplicação univalente que aplica $Y_{n}(i) \cup \alpha \cup Y_{n}(j)$ sobre $Y_{n}(1) \cup \eta \cup W$, no qual $\eta=P_{c}^{1-i}(\alpha)$ é o arco ligando $Y_{n}(1)$ ao $W=P_{c}^{1-i}\left(Y_{n}(j)\right)$.

Observemos que $W=Y_{j-i+1}$ ou é disjunto do conjunto pós-crítico de $P_{c}$. 
Já que o valor crítico $P_{c}(0) \in Y_{n}(1)$, o conjunto $W \cup \alpha \cup \partial Y_{n}(1)$ tem um recobrimento duplo por $P_{c}$ igual a $Z_{n} \cup \beta \cup \partial X_{n} \cup-\beta \cup-Z_{n}$ no qual $P_{c}\left(Z_{n}\right)=P_{c}\left(-Z_{n}\right)=W$ e $P_{c}(\beta)=P_{c}(-\beta)=\eta$.

Se $Z_{n}$ tem interseção não vazia com o conjunto pós-crítico, então, $-Z_{n}$ tem interseção vazia com $P(c)$, pois neste caso $Z_{n}=Y_{n}(j-1)$ e, portanto, $-Z_{n}=Y_{n}^{\prime}(j-1)$. Dessarte, trocandose, se se fizer necessário, $Z_{n}$ e $-Z_{n}$ podemos supor que $Z_{n}$ é disjunto de $\mathrm{P}(c)$. Isto estabelecido, teremos $P_{c}^{i}: Z_{n} \longrightarrow Y_{n}(j)$ univalente, mas sendo também $P_{c}^{n-j}: Y_{n}(j) \longrightarrow Y_{n}$ univalente segue que $P_{c}^{m}: Z_{n} \longrightarrow Y_{n}$ é univalente, em que $m=n+i-j<n$.

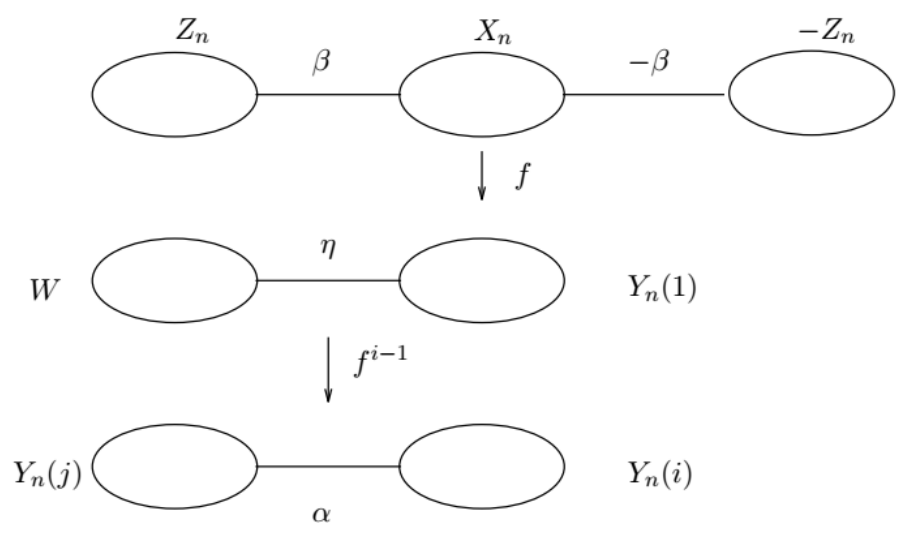

Figura 5.2: Construção de $Z_{n}$

A aplicação $\left.P_{c}^{m}\right|_{\widehat{\mathbb{C}}-\mathrm{P}(c)}$ é expansora na norma hiperbólica, o que implica em $l_{\mathbb{H}}\left(\partial Z_{n}\right) \leq l\left(\gamma_{n}\right)<\lambda$.

Por fim, mostraremos a existência de uma cota inferio para a área de $Z_{n}$, que depende apenas de $\lambda$.

A ideia consiste em mostrar que $P_{c}^{m}$ não é muito expansora próximo da curva $\partial Z_{n}$ e que mapeia uma vizinhança de $\partial Z_{n}$ sobre uma região com área definida em $Y_{n}$.

Para tanto, consideremos o seguite conjunto

$$
E:=\left\{z \in \widehat{\mathbb{C}}-\mathrm{P}(c) ; \mathrm{d}_{\mathbb{H}}\left(z, \gamma_{n}\right)<1\right\} \cap Y_{n}
$$

Seja $C\left(\gamma_{n}\right) \subset \widehat{\mathbb{C}}-\mathrm{P}(c)$ a vizinhança colar de $\gamma_{n}$ de $\gamma_{n}$ dada pelo teorema 2.18-[McM1]. Pondo $r(\lambda):=\mathrm{d}\left(\gamma_{n}, \partial C\left(\gamma_{n}\right)\right)$ e $r(z)$ o raio de injetividade de $\widehat{\mathbb{C}}-\mathrm{P}(c)$ em $z \in \gamma_{n}$ temos, $r(z) \geq r(\lambda)$ para todo $z \in \gamma_{n}$.

Temos a dependência de $r=\mathrm{d}\left(\gamma_{n}, \partial C\left(\gamma_{n}\right)\right)$ em $\lambda$ devido $r$ de $\bmod \left(C\left(\gamma_{n}\right)\right)$ que por sua vez depende de $l\left(\gamma_{n}\right)$ pelo teorema 2.19-[McM1], mas $n \in \mathrm{SR}(c, \lambda)$.

Sendo a aplicação $P_{c}^{n-j}: Y_{n}(j) \longrightarrow Y_{n}$ é univalente, assim existe uma região $E_{2} \subset Y_{n}(j)$ que é mapeada injetivamente sobre $E_{1} \subset Y_{n}$. Temos, $\left\|\left(P_{c}^{n-j}\right)^{\prime}(z)\right\|<C(\lambda)$ para todo $z \in \partial Y_{n}(j)$ pelo teorema 5.12 .

Para cada $z_{0} \in E_{2}$ considere o ponto $\widetilde{z}_{0} \in \partial Y_{n}(j)$ tal que $\mathrm{d}\left(z_{0}, \partial Y_{n}(j)\right)=\mathrm{d}\left(z_{0}, \widetilde{z}_{0}\right)$ e seja $\gamma$ a geodésica minimal em $\widehat{\mathbb{C}}-\mathrm{P}(c)$ que conecta $z_{0}$ ao $\widetilde{z}_{0}$. E disto, aplicando o teorema 3.8-[McM1], com tal curva $\gamma$, obtemos,

$$
\left\|\left(P_{c}^{n-j}\right)^{\prime}\left(z_{0}\right)\right\| \leq\left\|\left(P_{c}^{n-j}\right)^{\prime}\left(\widetilde{z}_{0}\right)\right\|^{\alpha}=C(\lambda)^{\alpha}
$$

em que $\alpha=\exp (C)$ para uma constante universal $C$.

A partir disto, sendo a área de $E_{1}$ limitada inferiormente, obtemos uma cota inferior dependendo 
apenas de $\lambda$ para área $\left(E_{2}\right)$, pois

$$
\begin{aligned}
\operatorname{área}\left(E_{1}\right)=\int_{P_{c}^{n-j}\left(E_{2}\right)=E_{1}}(\rho(z))^{2}|d z|^{2} & =\int_{E_{2}}\left\|\left(P_{c}^{n-j}\right)^{\prime}(z)\right\|(\rho(z))^{2}|d z|^{2} \\
& \leq(C(\lambda))^{\alpha} \int_{E_{2}}(\rho(z))^{2}|d z|^{2} \\
& =(C(\lambda))^{\alpha}\left(E_{2}\right)
\end{aligned}
$$

Sendo também injetora a aplicação $P_{c}^{i}: Z_{n} \longrightarrow Y_{n}(j)$ existe uma região $E_{3} \subset Z_{n}$ que é mapeada injetivamente sobre $E_{2}$. Qualquer ponto $z_{0} \in E_{3}$ pode ser conectado à $\partial X_{n}$ por um arco $\xi$ tal que $l\left(P_{c}^{i}(\xi)\right)<C(\lambda)$. Para termos tal desigualdade basta-nos tomar $\xi$ como a pré-imagem de $\beta_{1} \vee \beta_{2} \vee P_{c}^{i}(\beta)$, em que $\beta_{1}$ é a geodésica minimal em $\widehat{\mathbb{C}}$ conectando $P_{c}^{i}\left(z_{0}\right) \in E_{2}$ à $\partial Y_{n}(j)$ e $\beta_{2}$ é o arco de $\partial Y_{n}(j)$ conectando $\beta_{1} \cap \partial Y_{n}(j)$ a $\partial Y_{n}(j) \cap P_{c}^{i}(\beta)$. Já que $\beta_{1}$, $\beta_{2}$ e $P_{c}^{i}(\beta)$ possuem seu comprimentos limitados em termos de $\lambda$ na métrica hiperbólica de $\widehat{\mathbb{C}}$.

Do teorema 3.8-[McM1] e 5.12

$$
\left\|\left(P_{c}^{i}\right)^{\prime}\left(z_{0}\right)\right\| \leq\left\|\left(P_{c}^{i}\right)^{\prime}\left(z_{1}\right)\right\|^{\alpha} \leq(C(\lambda))^{\alpha}
$$

em que $z_{1}$ é o ponto $P_{c}^{i}(\beta) \cap Y_{n}(i)$. Mas $\alpha=\exp \left(C \cdot l\left(P_{c}^{i}(\xi)\right)\right)<\exp (C(\lambda))$. Portanto, $\left\|\left(P_{c}^{i}\right)^{\prime}\left(z_{0}\right)\right\| \leq$ $C(\lambda)$ para todo $z_{0} \in E_{3}$. Isto posto, obtemos área $\left(E_{2}\right)=C(\lambda)$ área $\left(E_{3}\right)$.

Logo, área $\left(Z_{n}\right)>\operatorname{área}\left(E_{3}\right)>\frac{\text { área }\left(E_{1}\right)}{C(\lambda)}>\frac{1}{C(\lambda)}$.

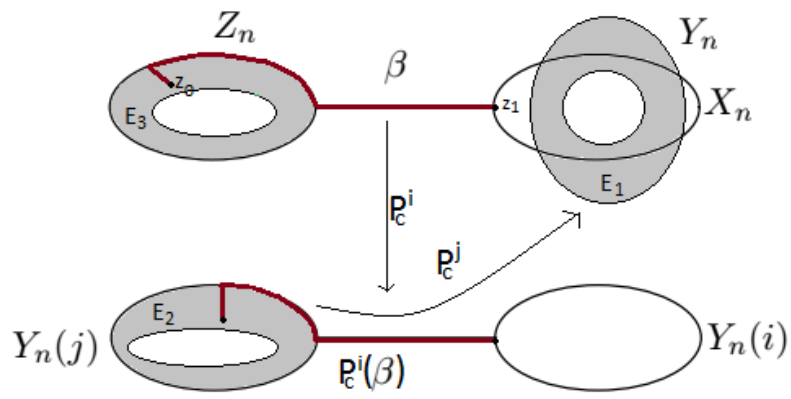

Figura 5.3: ilustração

Teorema 5.15 (Campo de linhas próprio invariante). Suponhamos $|\operatorname{SR}(c, \lambda)|=\infty$ e que $P_{c}$ admita um campo de linhas invariante $\mu$ suportado em seu Julia. Então, existe uma subsequência de niveis $n \in S R(c)$ tal que $\mu_{n}=\left(A_{n}^{-1}\right)^{*}\left(\left.\mu\right|_{Y_{n}}\right)$ converge para um campo de linhas univalente $\nu$ em $Y$ invariante por $P$, em que $A_{n}$ e $P$ são aquelas aplicaçôes do teorema 5.10 .

Demonstração. Seja $x \in \widehat{\mathbb{C}}-\mathrm{P}(c)$ um ponto quasecontinuidade de $\mu$ com as propriedades garantidas pelo corolário 5.3.

Para cada nível $n \in \mathrm{SR}(c, \lambda)$ definamos $k(n) \geq 0$ como sendo o menor inteiro tal que $P_{c}^{k(n)+1}(x) \in$ $y_{n}$. Por mesma razão dada na demonstração do teorema $5.5, k(n) \rightarrow+\infty$ quando $n \rightarrow+\infty$ em $\operatorname{SR}(c, \lambda)$. Consideremos apenas $n$ suficientemente grande de modo que $k(n)>0$ e assim tenhamos $P_{c}^{k(n)}(x) \notin y_{n}$.

Temos $P_{c}^{k(n)+1}(x) \in Y_{n}(i(n)+1)$ para algum $i(n)$ com $0 \leq i(n) \leq n-1$. Distinguiremos dois casos, o primeiro, quando $i(n)>0$ e o segundo, quando $i(n)=0$. 


\section{1o caso:}

$P_{c}^{k(n)}(x) \in Y_{n}^{\prime}(i(n))$, pois $P_{c}^{k(n)}(x) \notin y_{n}$ e $P_{c}^{k(n)+1}(x) \in y_{n}$. Definamos $h_{n}: Y_{n} \longrightarrow \mathbb{C}$ como segue

$$
Y_{n} \stackrel{P_{c}^{i(n)-n} \longmapsto}{\longmapsto} Y_{n}^{\prime}(i(n)) \stackrel{P_{c}^{-k(n)}}{\longmapsto} T_{n} \subset \mathbb{C}
$$

em que o ramo univalente de $P_{c}^{-k(n)}$ é tomado de modo que ele envie $P_{c}^{k(n)}(x)$ de volta em $x$. Este ramo está definido em todo $Y_{n}^{\prime}(i(n))$ já que este tem interseção vazia com $\mathrm{P}(c)$.

\section{$2^{\circ}$ caso:}

Neste caso $P_{c}^{k(n)+1} \in Y_{n}(1)$, e como $P_{c}^{k(n)+1} \notin Y_{n} \subset y_{n}$. Então, $P_{c}^{k(n)(x) \in X_{n}-Y_{n}}$, pois $P_{c}^{n}\left(P_{c}^{k(n)}\right) \in$ $Y_{n}$.

Como $\partial Y_{n}=\gamma_{n} \approx_{i s o t} \delta_{n}, X_{n}-Y_{n}$ é disjunto do conjunto pós-crítico $\mathrm{P}(c)$. Do corolário 5.4, $\delta_{n}=\partial X_{n}$ fica contida em uma $C(\lambda)$-vizinhança de $\gamma_{n}=\partial Y_{n}$, e o mesmo vale para a região $X_{n}-Y_{n}$. Em particular, a distância de $P_{c}^{k(n)}(x)$ à $\partial X_{n}$ é limitada. Pelo teorema 5.14, a distância de $\partial X_{n}$ à $\partial Z_{n}$ é limitada em termos de $\lambda$ e o comprimento de $\partial X_{n}$ é limitado por $2 \lambda$, do que conclui-se que $\mathrm{d}_{\mathbb{H}}\left(P_{c}^{k(n)}(x), Z_{n}\right) \leq C(\lambda)$.

Seja $\zeta_{n}$ a geodésica minimal ligando $P_{n}^{k(n)}$ ao $Z_{n}$. Como $\zeta_{n} \cup Z_{n}$ é disjunto do conjunto pós-crítico de $P_{c}$ existe um ramo univalente de $P_{c}^{-k(n)}$ em uma vizinhança de $\zeta_{n} \cup Z_{n}$ que envia $P_{c}^{k(n)}(x)$ em $x$ e $\zeta_{n}$ em um arco que conecta $x$ ao $T_{n}$, imagem de $Z_{n}$ por $P_{c}^{-k(n)}$.

Do item 1 do teorema 5.14 decorre a existência de um inteiro $m \in\{1,2, \cdots, n-1\}$ tal que $P_{c}^{m}: Z_{n} \longrightarrow Y_{n}$ é univalente. Com tudo isso, definamos a aplicação univalente $h: Y_{n} \longrightarrow T_{n}$ dada por

$$
Y_{n} \stackrel{P_{c}^{-m}}{\longmapsto} Z_{n} \stackrel{P_{c}^{-k(n)}}{\longmapsto} T_{n}
$$

Provemos a seguir dois lemas que nos auxiliarão na continuação desta prova.

Lema 5.1. Quando $n \rightarrow \infty$ em $\operatorname{SR}(c, \lambda)$,

$$
\operatorname{diâm}_{\mathbb{H}}\left(T_{n}\right) \rightarrow 0
$$

enquanto

$$
\mathrm{d}_{\mathbb{H}}\left(x, T_{n}\right) \leq C(\lambda) \operatorname{diâ}_{\mathbb{H}}\left(T_{n}\right)
$$

na métrica hiperbólica em $\widehat{\mathbb{C}}-\mathrm{P}(c)$.

prova do lema 5.1. Usaremos o fato de que $\left\|\left(P_{c}^{k(n)}\right)^{\prime}(x)\right\| \rightarrow+\infty$ quando $n \rightarrow+\infty$ (teorema 3.6[McM1]).

No $1^{\text {o }}$ caso, $P_{c}^{k(n)}$ mapeia $T_{n}$ injetivamente sobre $Y_{n}^{\prime}(i(n))$, enviando $x$ ao interior de $Y_{n}^{\prime}(i(n))$.

Visto que o diâmetro de $\partial Y_{n}^{\prime}(i(n))$ em $\widehat{\mathbb{C}}-\mathrm{P}(c)$ é limitado independentemente do nível $n$, $\left\|P_{c}^{k(n)}(z)\right\|$ tende uniformemente para o infinito em $\partial T_{n}$, como veremos a seguir. 
Sendo $D(\lambda):=\operatorname{diâm}_{\mathbb{H}}\left(Y_{n}^{\prime}(i(n))\right)$, do teorema 3.8-[McM1], temos para cada $z \in T_{n}$

$$
\left\|\left(P_{c}^{k(n)}\right)^{\prime}(z)\right\| \geq\left\|\left(P_{c}^{k(n)}\right)^{\prime}\left(z_{0}\right)\right\|^{\frac{1}{\alpha}}=M_{n}(\lambda),
$$

já que $Y_{n}^{\prime}(i(n))=P_{c}^{k(n)}\left(T_{n}\right)$, em que $\alpha=\exp (C D(\lambda))$ e $C$ é uma constante universal e $z_{0} \in \partial T_{n}$ é tal que $\left\|\left(P_{c}^{k(n)}\right)^{\prime}\left(z_{0}\right)\right\|=\min \left\{\left\|\left(P_{c}^{k(n)}\right)^{\prime}(z)\right\|, z \in \partial T_{n}\right\}$.

Como $\partial Y_{n}^{\prime}(i(n))=P_{c}^{k(n)}\left(\partial T_{n}\right)$, dos teoremas 3.5-[McM1] e $4.3, l_{\mathbb{H}}\left(\partial Y_{n}^{\prime}(i(n))\right) \leq \lambda$. E disto decorre,

$$
\begin{aligned}
\lambda \geq l_{\mathbb{H}}\left(\partial Y_{n}^{\prime}(i(n))\right) & =\int_{\partial Y_{n}^{\prime}(i(n))} \rho(z)|d z|=\int_{\partial T_{n}}\left\|\left(P_{c}^{k(n)}\right)^{\prime}(z)\right\| \rho(z)|d z| \\
& \geq M_{n}^{\alpha}(\lambda) l_{\mathbb{H}}\left(\partial T_{n}\right)
\end{aligned}
$$

Mas, $M_{n} \rightarrow+\infty$ quando $n \rightarrow+\infty$, logo deve-se ter, $l_{\mathbb{H}}\left(\partial T_{n}\right) \rightarrow 0$ e, em particular, $\operatorname{diâm}\left(T_{n}\right) \rightarrow$ 0 . Neste caso, a desigualdade é trivial, pois $x \in T_{n}$.

Passemos agora para o $2^{\mathbf{o}}$ caso, no qual $i(k)=0$ e $P_{c}^{k(n)}$ aplica $T_{n}$ de maneira univalente sobre $Z_{n}$

\section{Afirmação 5.1.}

$$
\frac{1}{C(\lambda)} \leq \frac{\left\|\left(P_{c}^{k(n)}\right)^{\prime}(z)\right\|}{\left\|\left(P_{c}^{k(n)}\right)^{\prime}(x)\right\|} \leq C(\lambda)
$$

para todo $z \in T_{n} \cup \tau_{n}$, no qual $\tau_{n}$ é um arco que conecta $x$ ao $T_{n}$.

Prova da Afirmação 5.1. Pelo teorema da vizinhança colar o raio de injetividade de $\widehat{\mathbb{C}}-\mathrm{P}(c)$ em $\gamma_{n}$ é limitado inferiormente em termos de $\lambda$. E temos ainda $l_{\mathbb{H}}\left(\gamma_{n}\right)>\frac{1}{\lambda}$.

Isto posto, recorrendo ao teorema 2.22-[McM1] que nos atesta que o logarítmo do raio de injetividade é uniformemente Lipschitz, teremos para todo $z \in \gamma_{n}$

$$
\begin{aligned}
\log \left(r\left(P_{c}^{k(n)}(x)\right)\right) & \geq \log (r(z))-C \mathrm{~d}_{\mathbb{H}}(x, z) \\
& \geq \log (C(\lambda))-C\left[\mathrm{~d}_{\mathbb{H}}(x, \widehat{x})+\mathrm{d}_{\mathbb{H}}(\widehat{x}, z)\right] \\
& \geq \log (C(\lambda))-C\left(C(\lambda)+\frac{1}{\lambda}\right):=K(\lambda),
\end{aligned}
$$

em que $\widehat{x}$ é o ponto sobre $\gamma_{n}$ tal que $\mathrm{d}_{\mathbb{H}}(x, \widehat{x})=\mathrm{d}_{\mathbb{H}}\left(x, \gamma_{n}\right)$, portanto,

$$
r\left(P_{c}^{k(n)}(x)\right) \geq K(\lambda)>0
$$

Podemos ligar $z \in T_{n} \cup \tau_{n}$ a $x$ por um caminho $\xi$ em $\widehat{\mathbb{C}}-\mathrm{P}(c)$ tal que $l_{\mathbb{H}}\left(P_{c}^{k(n)}(\xi)\right) \leq l_{\mathbb{H}}\left(\zeta_{n}\right)+$ $\operatorname{diâm}_{\mathbb{H}}\left(Z_{n}\right)$. Bastando para tanto, fazermos o seguinte:

1. se $z \in \tau_{n}$ tomamos $\xi$ como sendo o subarco de $\tau_{n}$ que conecta $z$ ao $x$;

2. se $z \in \partial T_{n}$, então, $\xi$ será a justaposição de $\tau_{n}$ com o sub-arco de $\partial T_{n}$ que conecta $z$ ao ponto $\partial T_{n} \cap \tau_{n} ;$

3. se $z \in T_{n}$, $\xi$ será a justaposição $\alpha \vee \tau$, na qual $\alpha$ é a geodéca minimal que conecta $z$ ao ponto $\partial T_{n} \cap \tau_{n}$. 
Nos três casos acima é imediato que

$$
C(\lambda) \geq \operatorname{diâm}_{\mathbb{H}}\left(Z_{n}\right)+l_{\mathbb{H}}\left(\zeta_{n}=P_{c}^{k(n)}\left(\tau_{n}\right)\right) \geq l_{\mathbb{H}}\left(P_{c}^{k(n)}(\xi)\right)
$$

Pois, $\operatorname{diâm}_{\mathbb{H}}\left(Z_{n}\right)$ é limitado em termos de $\lambda$, pois $P_{c}^{m}\left(Z_{n}\right)=Y_{n}$ com $\left.P_{c}^{m}\right|_{Z_{n}}$ univalente, o que é assertado pelo teorema 5.14.

A partir disto, aplicando o teorema 3.8-[McM1], obtemos

$$
C(\lambda)^{-1} \leq \frac{\left\|\left(P_{c}^{k(n)}\right)^{\prime}(z)\right\|}{\left\|\left(P_{c}^{k(n)}\right)^{\prime}(x)\right\|} \leq C(\lambda),
$$

em que a dependência de $C(\lambda)$ a $\lambda$ decorrem de 5.78 e 5.79 .

De 5.80 segue

$$
\mathrm{d}_{\mathbb{H}}(x, z) \leq \int_{\xi}\left\|\left(P_{c}^{k(n)}\right)^{\prime}(z)\right\| \rho(z)|d z| \leq \frac{C(\lambda)}{\left\|\left(P_{c}^{k(n)}\right)^{\prime}(x)\right\|},
$$

para qualquer $z \in T_{n}$.

Desde que, $\left\|\left(P_{c}^{k(n)}\right)^{\prime}(x)\right\| \rightarrow+\infty$ quando $n \rightarrow+\infty$, diâm $\mathbb{H}\left(T_{n}\right) \rightarrow 0$.

Para concluir, mostraremos que $\mathrm{d}_{\mathbb{H}}\left(x, T_{n}\right) \leq C(\lambda) \operatorname{diâm}_{\mathbb{H}}\left(T_{n}\right)$

Do item 4 do teorema 5.14 sabemos que a área de $Z_{z}$ é limitada inferiormente em termos de $\lambda$, assim,

$$
\begin{aligned}
\frac{1}{C(\lambda)} \leq \operatorname{área}\left(Z_{n}\right) & =\int_{Z_{n}=P_{c}^{k(n)}} \rho(z)^{2}|d z|^{2}=\int_{T_{n}}\left\|\left(P_{c}^{k(n)}\right)^{\prime}(z)\right\|^{2} \rho(z)^{2}|d z|^{2} \\
\text { (usando 5.80) } & \leq \widetilde{C}(\lambda)\left\|\left(P_{c}^{k(n)}\right)^{\prime}(x)\right\|^{2} \int_{T_{n}} \rho(z)^{2}|d z|^{2} \\
& =\widetilde{C}(\lambda)\left\|\left(P_{c}^{k(n)}\right)^{\prime}(x)\right\|^{2} \text { área }\left(T_{n}\right)
\end{aligned}
$$

Sendo o diâmetro de $T_{n}$ limitado na métrica hiperbólica de $\widehat{\mathbb{C}}-\mathrm{P}(c)$ em termos de $\lambda$, temos, área $\left(T_{n}\right) \leq C$ diâm $\left(T_{n}\right)^{2}$ para uma constante $C>0$. Com isso, de 5.82 acima obtemos

$$
\frac{1}{\left\|\left(P_{c}^{k(n)}\right)^{\prime}(x)\right\|^{2}} \leq C C(\lambda) \widetilde{C}(\lambda)\left(\operatorname{diâm}_{\mathbb{H}}\left(T_{n}\right)\right)^{2}
$$

e, portanto,

$$
\frac{1}{\left\|\left(P_{c}^{k(n)}\right)^{\prime}(x)\right\|^{2}} \leq C(\lambda)\left(\operatorname{diâm}_{\mathbb{H}}\left(T_{n}\right)\right)^{2}
$$

Então, de 5.81

$$
\left.\mathrm{d}_{\mathbb{H}}\left(x, T_{n}\right)\right) \leq \mathrm{d}_{\mathbb{H}}(x, z) \leq \frac{C(\lambda)}{\left\|\left(P_{c}^{k(n)}\right)^{\prime}(z)\right\|} \leq C(\lambda) \operatorname{diâm}_{\mathbb{H}}\left(T_{n}\right)
$$

Lema 5.2. A aplicação $h_{n}$ se estende a uma aplicação univalente em um anel com módulo definido em torno de $Y_{n}$. 
Demonstração. O teorema da vizinhança colar nos garante a existência de um anel $C\left(\gamma_{n}\right) \subset$ $\widehat{\mathbb{C}}-\mathrm{P}(c)$ com módulo definido cuja curva central é $\gamma_{n}$.

Assim, qualquer aplicação univalente em $Y_{n}$ se estende univalentemente à região $C\left(\gamma_{n}\right) \cup Y_{n}$. Portanto, sendo $h_{n}$ um ramo da inversa de alguma iterada de $P_{c}$, temos o esperado.

Processeguimos a prova do teorema considerando uma sequência de níveis $n(k) \in \operatorname{SR}(c, \lambda)$ para a qual valha o teorema 5.10. Lembre-se que, em particular, temos $\left(A_{n}\left(Y_{n}, 0\right)\right) \rightarrow(Y, 0)$ na topologia de Carathéodory.

Ponhamos,

$$
k_{n}:=h_{n} \circ A_{n}^{-1}: A_{n}\left(Y_{n}\right) \longrightarrow T_{n}
$$

Aplicando o teorema 5.8 , já que $\operatorname{diâm}_{\mathbb{E}}\left(A_{n}\left(Y_{n}\right)\right)=\frac{\operatorname{diâm}_{\mathbb{E}}\left(Y_{n}\right)}{\operatorname{diâm}_{\mathbb{E}}\left(B_{n}\right)}$, obtemos

$$
1 \leq \operatorname{diâm}_{\mathbb{E}}\left(A_{n}\left(Y_{n}\right)\right) \leq \frac{1}{C(\lambda)}:=\widetilde{C}(\lambda)
$$

O lema 5.2 nos assegura que $k_{n}$ admite uma extensão univalente a um anel com módulo definido em torno de $A_{n}\left(Y_{n}\right)$ e $k_{n}\left(A_{n}\left(Y_{n}\right)\right)=T_{n}$. Disto mais o teorema $3.8-$ [McM1] concluímos

$$
\frac{1}{C(\lambda)}\left\|k_{n}^{\prime}(0)\right\| \leq \operatorname{diâm}_{\mathbb{H}}\left(T_{n}\right) \leq C(\lambda)\left\|k_{n}^{\prime}(0)\right\|
$$

Visto que as norma euclidiana e a norma hiperbólica são comparáveis numa vizinhança de $x$ e $\operatorname{diâm}_{\mathbb{H}}\left(T_{n}\right)$ tende para $0,\left|k_{n}^{\prime}(0)\right| \rightarrow 0$ quando $n \rightarrow \infty$.

Desde que $k_{n}(0) \in T_{n}$ e $\frac{1}{\left\|k_{n}^{\prime}(0)\right\|} \geq \frac{1}{C(\lambda) \operatorname{diâm}_{\mathbb{H}}\left(T_{n}\right)}$ por 5.88 , segue

$$
\frac{\operatorname{diâm}_{\mathbb{H}}\left(x, k_{n}(0)\right)}{\left\|k_{n}^{\prime}(0)\right\|} \leq \frac{\mathrm{d}_{\mathbb{H}}\left(x, T_{n}\right)+\operatorname{diâ}_{\mathbb{H}}\left(T_{n}\right)}{C(\lambda) \operatorname{diâm}_{\mathbb{H}}\left(T_{n}\right)}
$$

O lema 5.1 implica que $\frac{\mathrm{d}_{\mathbb{H}}\left(x, T_{n}\right)+\operatorname{diâm}_{\mathbb{H}}\left(T_{n}\right)}{C(\lambda) \operatorname{diâm}_{\mathbb{H}}\left(T_{n}\right)}$ é limitado em termos de $\lambda$. Logo, pelo teorema 5.16-[McM1] existe uma subsequência de níveis $n \in \mathrm{SR}(c, \lambda)$ tal que

$$
\nu_{n}=k_{n}^{*}(\mu)
$$

converge em medida para um campo de linhas univalente $\nu$ em $(Y, 0)$. Mas, $h_{n}$ é um ramo inverso de alguma iterada de $P_{c}$, assim a invariância de $\mu$ por $P_{c}$ implica que $\nu_{n}=\left(A_{n}^{-1}\right)^{*}(\mu)$. Mais ainda,

$$
\begin{aligned}
\left(A_{n} \circ P_{c}^{n} \circ A_{n}^{-1}\right)^{*}\left(A_{n}^{-1}\right)^{*}(\mu) & =\left(A_{N}^{-1}\right)^{*}\left(P_{c}^{n}\right)^{*}\left(A_{n}\right)^{*}\left(A_{n}^{-1}\right)^{*}(\mu) \\
& =\left(A_{N}^{-1}\right)^{*}\left(P_{c}^{n}\right)^{*}(\mu) \\
& =\left(A_{N}^{-1}\right)^{*}(\mu) \\
& =\nu_{n}
\end{aligned}
$$

Desta maneira, aplicando o teorema 5.14-[McM1] concluímos que $\nu$ é invariente por $P$ em $Y$. Não obstante, $P:(X, 0) \longrightarrow(Y, P(0))$ tem $z=0 \in Y$ como ponto crítico, logo pelo teorema 5.3-[McM1], $P$ não pode admitir um campo de linhas univalente invariante. 
Portanto, $P_{c}$ não admite um campo de linhas invarinte suportado em seu conjunto de Julia. prova do teorema 5.7. Como comentado na definição 5.4, temos $|\operatorname{SR}(c, \lambda)|=+\infty$. Portanto, do teorema 5.15, temos a prova de 5.7.

Por conseguinte, juntando o teorema 5.7 com o corolário 5.2 obtemos o teorema de rigidez robusta 5.1 . 


\section{Capítulo 6}

\section{Considerações finais}

A teoria de renormalização tem sido de fundamental importância na dinâmica holomorfa devido à sua forte ligação com os principais problemas em aberto e com as frentes de pesquisas nesta área(veja [Fa],[Shi] mais referências aí contidas). Portanto, esta dissertação faz parte de uma introdução à novas técnicas e um aprofundamento no entendimento dos resultados básicos da dinâmica holomorfa. E que tem um caráter convidativo às pesquisas neste contexto devido às possíveis generalizações, haja vista que no presente trabalho apenas parte da rigidez quaseconforme é provada.

Lyubich [L1] provou a Conjectura 1.0.1 para a classe dos polinômios quadráticos infinitamente renormalizados de tipo limitado. Kozlovski, Shen e van Strien em [KSvS] obtiveram a Conjectura 1.0.1 para polinômios reais com pontos críticos reais. Avila, et al. em [AKLS] provaram a Conjectura 1.0.1 para qualquer polinômio unicrítico de grau qualquer que seja no máximo finitamente renormalizável e sem pontos periódicos não repulsores. No contexto geral das funções racionais este problema foi completamente resolvido por Yu Zhai e Yongcheng Yin em [YZ] e [Zh] para aplicações racionais cujo conjunto de Julia é um Cantor. Hiroyuki Inou em [In] generaliza o Teorema 1.1 de McMullen provando que Um polinômio infinitamente renormalizável robusto não suporta em seu Julia um campo de linhas invariante. Recentimente, Wenjuan Peng e Tan Lei em [PT] obtiveram a rigidez quaseconforme para aplicações multicríticas. 


\section{Referências Bibliográficas}

[Ah1] AHLFORS, Lars, Complex Analysis: An Introduction to the Theory of Analytic Functions of One Complex Variable . 3th. ed. New York: McGraw-Hill Book Company, (1987).

[Ah2] AHLFORS, Lars. V. Lectures on quasiconformal mappings. second ed., University Lecture Series, vol. 38, American Mathematical Society, Providence, RI, 2006.

[Av] AVILA, Artur. Dynamics of Renormalization Operator. Proceedings of International Congrees of Mathemathicians.Hyderabad.India.(2010).

[AKLS] AVILA, Artur; KAHN, J.; LYUBICH, M.; SHEN, W. Combinatorial rigidity for unicritical polynomials. Annals of Mathematics, 170 (2009), 783-797. 73

[AL] AVILA, Artur, LYUBICH, Mikhail. The full renormalization horseshoe for unimodal maps of higher degree: exponential contraction on hybrid classes. Publ. Math. Inst. Hautes Étude Sci. 114 (2011),p.171-223. 31

[ALM] AVILA, Artur; Lyubich, M. and de Melo, W. Regular or stochastic dynamics in real analytic families of unimodal maps. Invent. Math., 154 (2003), 451-550.

[B1] BOURBAKI, Nicolas. Elements of Mathematics: theory of sets. Springer. (2004),p.191209.

[B2] BOURBAKI, Nicolas. Elements of Mathematics: Algebra I: chapters:1-3. SpringerVerlag. (2004),p.118-123.

[BrFa] BRANNER, Bodil; FAGELA, Núria. Quasiconformal Surgery in holomorfphic dynamics. Cambridge Studies in Advanced Mathematics. Cambridge University Press. New York. 141. 1st ed. (2014).

[BrH] BRANNER, B.;HUBBARD, J. H. The iteration of cubic polynomials, Part II: patterns and parapatterns, Acta Math.169(1992),p.229-325. 44

[CG] CARLeSON, L.; GAMELIN, T. Complex Dynamics. Spriger-Verlag. New York. (1993). 23

[DH1] DOUADY, Adrien; HUBBARD, John H. Étude dynamique des polynômes complexes. Pub. Math. d'Orsay. 2nd. ed. (1984).

[DH2] DOUADY, Adrien; HUBBARD, John H. Exploring the Mandelbrot set. Pub. Math. d'Orsay. 2nd. ed. (1985). 
[DH3] DOUADY, Adrien; HUBBARD, John H. On the dynamics of polynomial-like mappings. Sci. Èc. Norm. Sup. 18(1985). p. 287-344.

[Dou1] DOUADY, Adrien. Systèmes dynamiques holomorphes. Bourbaki seminar. vol. 1982/83. Astérisque. Soc. Math. France, Paris. vol.105-106(1983). p.39-64.

[Dou2] DOUADY, Adrien. Compacts in C. Topological methods in modern mathematics. Publish or Perish, Houston (1992), p.429-465. 15

[Fa] FAGELA, Núria. Notes on Renormalization of Complex Polynomials. Renormalizations, proceedings of a workshop. IHP.(1996). ed. Sylvie Payschapp. p.40-51. 73

[FM] FARB, Benson; MARGALIT, Dan A primer on mapping class groups. Princeton University Press. Princeton, NJ 2012.

[Hu] HUBBARD, J. H., et al. Teichmüller theory and applications to geometry, topology, and dynamics.. Vol. 1. Matrix Editions. Ithaca. NY. 2006. 21, 29

[In] INOU, Hiroyuki. Renormalization and rigidity of polynomials of higher degree.. J. Math. Kyoto Univ. 42-2. (2002). p.351-392. 73

$[J]$ JIANG, Yunping. Renormalization and geometry in one-dimensional real and complex dynamics. Advanced Series in nonlinear dynamics.vol.10.World Scientific.New York. 1996. 44

[Jen] JENKINS, James A. On weighted distortion in conformal mapping.II. Bull. London Math. Soc.V.30.no.2.1998.p.151-158. 58

[KSvS] KOZLOVSKI, O.; SHEN, W. and van STRIEN, S. Rigidity for real polynomials. Ann. of Math. (2) 165 (2007), no. 3, p.749-841. 73

[L1] LYUBICH, M. Dynamics of quadratic polynomials. I, II. Acta. Math. 178 (1997), n. 2, p.185-247, p.247-297. 73

[L2] LYUBICH, M. Feigenbaum-Coullet-Tresser universality and Milnor's hairiness conjecture. Ann. Math. (2) 149 (1999). p.319-420. 31

[L3] LYUBICH, M. Book Riviews. Bull. (new series) of the Amer. Math. Soc. vol.36. n.1.(1999). p.103-107.

[L4] LYUBICH, M. Six Lectures on Real and Complex Dynamics. disponivel em: $<$ https://www.math.stonybrook.edu/ mlyubich/Archive/Selected/lectures.pdf $>31$

[McM1] MCMUllen, Curtis T. Complex Dynamics and Renormalization. Annals of Mathematics studies: no. 135.Princeton University Press, 1994. iii, v, 6, 16, 17, 24, 25, 31, 32, 34, 35, $38,39,40,44,48,50,54,55,56,57,58,59,61,62,63,64,65,66,67,68,69,70$

[McM2] MCMULLEN, Curtis T. Renormalization and 3-manifold with fiber over the circle. Annals of Mathematics studies: no. 142.Princeton University Press, 1996. 41 
[McM3] MCMUlLEN, Curtis T. Frontiers in complex dynamics. Bull. Amer. Math. Soc. (N.S.) 31 (1994), no. 2, p.155-172.

[Mil1] MILNOR, John. Dynamics in one complex variable, 3rd edition. Annals of Mathematics studies series n.160, Princeton University Press, 2006. 3, 16, 17, 20, 23, 27, 34, 42, 47, 54

[Mil2] MILNOR, John. Local connectivity of the Julia sets:expository lectures. in The Mandelbrot Set, Theme and Variations, Edit. Tan Lei. Cambridge U. Press, Cambridge, UK, 67-116, 2000. ix, 41, 44

[MSS] MAÑÉ, R.; SAD, P.; SULLIVAN, D. On the dynamics of rational maps. Ann. Sci. Éc. Norm. Sup. 16(1983). P. 193-217. 23, 24, 25

[PT] PENG, Wenjuan;LEI, Tan. Quasi-Conformal Rigidity of Multicritical Maps. Sci. China Ser. A. (2008). n.58. p.79-92. 73

[R] REMMERT, R., Theory of Complex Functions.Graduate Texts in Mathematics.v.122 New York: Springer-Verlag, 1991. 58, 62

[Ru] RUDIN, W., Real and Complex Analysis . 3th. ed. New York: McGraw-Hill Book Company, 1987.

[Sch] SCHLEICHER, D. On fibers and renormalization of Julia sets and Multibrot sets in Geometry and Applications:A Jubilee of Benoit Mandelbrot. vol.1, Proc. Sympos. Pure Math. 72, Amer. Math. Soc., Providence, RI, 2004.( and Preprint IMS at Stony Brook 13(1998). p. 477-517.)

[Shi] SHISHIKURA, Matisuhiro. Topological, Geometric and Complex Analytic Properties of Julia sets. Proceedings of International Congrees of Mathematicians. vol.1,2. Zürich. (1994). p.886-895. 73

[Sl] SLODKOWSKI, Z. Holomorphic motions and polynomial hulls. Proc. Am. Math. Soc., 111 (1991). p. $347-355.25$

[TY] TAN, Lei; YIN, Yongcheng. The unicritical Branner-Hubbard conjecture. Complex dynamics, A K Peters, Wellesley, MA, 2009. p. 215-227 44

[YZ] YIN, Yongcheng; ZHAI, Yu. No invariant line felds on Cntor Julia sets. Forum Mathematicum. 22 (290). n.1. p.75-94. 73

[Zh] ZHAI, Yu. Rigidity of rational maps with Cantor Julia sets. Sci. China Ser. A. (2008). n.58. p.79-92. 73 